\title{
Limit theorems in the stadium billiard
}

\author{
Péter Bálint* and Sébastien Gouëzel ${ }^{\dagger}$
}

October 8, 2018

\begin{abstract}
We prove that the Birkhoff sums for "almost every" relevant observable in the stadium billiard obey a non-standard limit law. More precisely, the usual central limit theorem holds for an observable if and only if its integral along a one-codimensional invariant set vanishes, otherwise a $\sqrt{n \log n}$ normalization is needed. As one of the two key steps in the argument, we obtain a limit theorem that holds in Young towers with exponential return time statistics in general, an abstract result that seems to be applicable to many other situations.
\end{abstract}

\section{Introduction}

The subject of this article, the stadium billiard, belongs to the class of dynamical systems that are sometimes referred to as intermittent ones. This name is related to the weakly chaotic nature of the time evolution that accounts for a modified, relaxed appearance of the behavior characteristic to systems with uniform hyperbolicity. In particular, the mathematically rigorous investigation of the stadium started with Bun79] where Bunimovich showed (with respect to the natural invariant measure) that the Lyapunov exponents are almost everywhere non-zero, and that the system is ergodic. Thus in that respect the stadium billiard resembles dispersing billiards, however, when finer statistical properties are discussed, deviations start to show up. Recent works by Markarian ([Mar04]) and Chernov-Zhang ([CZ]) have obtained an upper bound on the rate of mixing: given two sufficiently smooth (Hölder or Lipschitz continuous) observables, their correlations decay as $O\left((\log n)^{2} / n\right)$. Although this upper bound is most likely not sharp, it is definitely not far from the optimal either (see Corollary 1.2). In this paper we investigate the issue of probabilistic limit laws and provide further evidence of the intermittent nature of the dynamics. Namely we show that the limit behavior of a sufficiently smooth observable with zero mean, to be denoted by $f_{0}$, is characterized by a quantity $I$ (cf. (II)), its average along the one dimensional set of trajectories bouncing forever along the straight segments. In the typical case $I \neq 0$, the Birkhoff sums of $f_{0}$ satisfy a non-standard limit theorem - convergence in distribution to the Gaussian law can be obtained with a $\sqrt{c n \log n}$ normalization, where the constant $c$ is a multiple of $I^{2}$, see Theorem 1.1. On the other hand the central limit theorem in its usual form applies if $I=0$, see Theorem 1.3. These results

*Address: Institute of Mathematics, Budapest University of Technology and Economics, H-1111 Egry József u. 1, Budapest, Hungary; Email: bp@renyi.hu; URL: http://www.renyi.hu/ bp/

${ }^{\dagger}$ Address: Département de Mathématiques et Applications, Ecole Normale Supérieure, 45 rue d'Ulm, Paris, France; Email: Sebastien.Gouezel@ens.fr; URL:/http://www.dma.ens.fr/ gouezel/ 
have some almost immediate corollaries: we obtain the analogous limit theorems for the billiard flow (Corollary 1.4) and, though in a very weak form, some lower bounds on the rate of correlation decay (Corollary 1.2).

The issue of probabilistic limit laws in dynamical systems has a long history. In the chaotic setting the possibly most frequently applied method is Gordin's martingale argument (see [Gor69], or [You98 and references therein) that roughly states that under quite general conditions, whenever the correlations decay at a summable rate, the usual central limit theorem holds. This technique, however, cannot treat non-standard limit behavior or non-summable decay rates. Recently Aaronson and Denker have proposed an approach to the issue of non-standard limit theorems, see e.g. AD01. The dynamical systems they study, the so called Gibbs-Markov maps, possess some important features characteristic to uniformly expanding Markov maps of the interval, in particular, they are strongly chaotic. However, the functions $f$ for which limit theorems are proved are unbounded, and do not even belong to $L^{2}$. This setting allows for the use of Perron-Frobenius techniques: there is a one parameter family of transfer operators the spectra of which give precise information on the limit behavior of the observable. In particular, the Birkhoff sums satisfy exactly the same limit theorem that an i.i.d. sequence of random variables with the distribution of $f$ would have. For details see [AD01 and Section 3.1 of the present paper.

The above ideas can be implemented to treat limit laws for bounded functions in weakly chaotic systems $T_{0}: X_{0} \rightarrow X_{0}$ in case the following scenario applies. Let us assume that the source of non-uniformity in hyperbolicity is a well-distinguishable geometric effect. Then one may consider a subset $X \subset X_{0}$ such that the first return map onto $X$ is uniformly hyperbolic, however, our observable induces an unbounded function on $X$. Thus we arrive at a setting close to that of AD01. This line of approach has been successfully applied to systems for which the induced map is Gibbs-Markov (see eg. Gou04]), which, however, is not exactly the case of the stadium billiard.

What replaces Gibbs-Markov property in billiards is the presence of a Young tower, an object that has turned out to be very effective when estimating the rate of the decay of correlations. There are two versions of Young towers: those with exponential return time statistics ensure rapid mixing - exponential decay of correlations - via Perron-Frobenius techniques ([You98]), while those with polynomial return time statistics give polynomial upper bounds on the rate of correlation decay slow mixing rates - via coupling techniques ([You99]). As to the case of the stadium billiard, the Young towers constructed in Mar04 and [CZ have polynomial return time statistics with respect to the original map, and exponential return time statistics with respect to the induced map. The aim of the present paper is, in addition to present our results on the stadium billiard, to demonstrate that Young towers, originally designed to estimate mixing rates, are almost equally powerful when the issue of various limit laws is investigated. Note that this fact has already been observed and emphasized by Szász and Varjú in the papers [SV04a] and [SV04b].

The proof of Theorem 1.1 consists of two clearly distinguishable ingredients. On the one hand, via Perron-Frobenius techniques, we prove Theorem [3.4 a general result in Young towers with exponential return time statistics. This concerns the limit behavior of the Birkhoff sums of observables belonging to the non-standard domain of attraction of the Gaussian law. It is important to note that, as the Gibbs-Markov property is replaced by a Young tower, a new effect shows up that typically rescales the normalizing sequence with a constant multiplicator. We would also like to emphasize that this first ingredient of the proof is completely general and could be applied to many other situations. On the other hand, the second ingredient is directly related to the stadium billiard. We rely on Mar04 and [CZ when considering a suitable induced map that allows for a Young tower with exponential return time statistics. However, in order to "pull back" the limit theorem from the Young 
tower to the phase space of the billiard, and in order to give a transparent interpretation in terms of quantities easy to calculate, we need to perform a finer and more detailed geometric analysis of the stadium than the one presented in the above two papers.

We strongly do believe that our line of approach could be applied to obtain non-standard limit theorems in many other hyperbolic dynamical systems, in particular, in certain billiards with slow mixing rates. One of the most interesting candidates, the infinite horizon Lorentz process, for which the significance of the limit behavior is further emphasized as it may give an effective tool to discuss recurrence properties, is investigated by Szász and Varjú ([SV]). Among others, it is also worth mentioning skewed stadia (see [CZ]) and dispersing billiards with cusps ([Mac83]). We plan to turn back to these systems in separate papers.

The article has five sections. In the first one we state our main results and fix some basic notation. The second section is devoted to general results on the stadium billiard. We essentially recall the existence of Young towers for an induced map, proved by Markarian in [Mar04]. In the third part, we study abstract Young towers and establish a spectral perturbation estimate. In particular, to get a limit theorem, it is sufficient to study an integral with sufficient precision. In Section 4, we come back to the stadium billiard map, and describe geometrically this integral. With a careful study of the singularities of the stadium map, this gives an accurate description of this integral. Finally, in Section 5, we use together the abstract results of Section 3 and the explicit estimate of Section 4, to prove Theorem 1.1.

\section{Results}

Let $\ell>0$. We consider a region in the plane delimited by two semicircles of radius 1 , joined by two horizontal segments of length $\ell$, tangent to the semicircles. To a point on the boundary of this set and a vector pointing inwards, we associate an image by the usual billiard reflection law. This defines the stadium billiard map $T_{0}: X_{0} \rightarrow X_{0}$. This map admits a unique absolutely continuous invariant probability measure $\mu_{0}$.

A point in the phase space $X_{0}$ is given by $(r, \theta)$, where $r \in \mathbb{R} /(2 \pi+2 \ell) \mathbb{Z}$ is the position on the boundary, and $\theta \in(-\pi / 2, \pi / 2)$ is the angle with respect to the normal to this boundary at $r$. The invariant measure $\mu_{0}$ is given by

$$
\mathrm{d} \mu_{0}=\frac{\cos \theta \mathrm{d} r \mathrm{~d} \theta}{2(2 \pi+2 \ell)}
$$

We will assume that $r=0$ corresponds to the lower endpoint of the right semi-circle, and that the boundary is oriented counterclockwise. Hence, the semicircles correspond to $0 \leqslant r \leqslant \pi$ and $\pi+\ell \leqslant r \leqslant 2 \pi+\ell$.

Let $f_{0}: X_{0} \rightarrow \mathbb{R}$ be a Hölder function. We will be interested in the asymptotic behavior of the Birkhoff sums of $f_{0}$. The map $T_{0}$ is slowly mixing, by Mar04 and [CZ: its correlations decay (at least) like $O\left((\log n)^{2} / n\right)$. This estimate is not summable, whence the usual Gordin martingale argument to get a central limit theorem does not apply. We will indeed prove that the usual central limit theorem does not hold.

Let

$$
I=\frac{1}{2 \ell}\left[\int_{r \in[\pi, \pi+\ell]} f_{0}(r, 0) \mathrm{d} r+\int_{r \in[2 \pi+\ell, 2 \pi+2 \ell]} f_{0}(r, 0) \mathrm{d} r\right] .
$$


This is the average of $f_{0}$ along the trajectories bouncing perpendicularly to the segments of the stadium.

In this article, we prove the following theorem:

Theorem 1.1. Let $f_{0}: X_{0} \rightarrow \mathbb{R}$ be Hölder continuous, satisfying $\int f_{0} \mathrm{~d} \mu_{0}=0$ and $I \neq 0$. Then

$$
\frac{\sum_{k=0}^{n-1} f_{0} \circ T_{0}^{k}}{\sqrt{c n \log n}} \rightarrow \mathcal{N}(0,1)
$$

where

$$
c=\frac{4+3 \log 3}{4-3 \log 3} \cdot \frac{\ell^{2} I^{2}}{4(\pi+\ell)}
$$

Corollary 1.2. Under the assumptions of Theorem 1.1, the quantity $n \int f_{0} \cdot f_{0} \circ T_{0}^{n}$ does not tend to zero.

Proof. We have

$$
\int\left[\sum_{k=0}^{n-1} f_{0} \circ T_{0}^{k}\right]^{2}=n \int f_{0}^{2}+2 \sum_{i=1}^{n-1}(n-i) \int f_{0} \cdot f_{0} \circ T^{i} .
$$

If $\int f_{0} \cdot f_{0} \circ T^{i}=o(1 / i)$, we obtain $\int\left[\sum_{k=0}^{n-1} f_{0} \circ T_{0}^{k}\right]^{2}=o(n \log n)$. In particular, the variance of the random variable $\frac{\sum_{k=0}^{n-1} f_{0} \circ T_{0}^{k}}{\sqrt{n \log n}}$ tends to zero. This implies that this random variable tends to zero in probability, which is in contradiction with Theorem 1.1

Hence, we obtain a lower bound $O(1 / n)$ on the speed of decay of correlations of Hölder functions. It indicates that the upper bound of Markarian and Chernov-Zhang is close to optimal (it may probably be replaced by $O(1 / n)$, since the $(\log n)^{2}$ seems to be due to the technique of proof).

We also obtain the following (easier) result:

Theorem 1.3. Let $f_{0}: X_{0} \rightarrow \mathbb{R}$ be Hölder continuous, satisfying $\int f_{0} \mathrm{~d} \mu_{0}=0$ and $I=0$. Then there exists $\sigma^{2} \geqslant 0$ such that

$$
\frac{\sum_{k=0}^{n-1} f_{0} \circ T_{0}^{k}}{\sqrt{n}} \rightarrow \mathcal{N}\left(0, \sigma^{2}\right)
$$

Hence, when $I=0$, the Birkhoff sums of $f_{0}$ satisfy a usual central limit theorem.

Before going into the details of the proof we consider one particularly interesting observable: the free path. Given $x=(r, \theta)$, we denote $T_{0} x=\left(r_{1}, \theta_{1}\right)$ and define $\tau(x)$ as the planar distance of $r$ and $r_{1}$. In other words, $\tau(x)$ is the length of the trajectory segment the point particle follows until the next collision. To investigate the limit behavior of the free path $\tau: X_{0} \rightarrow \mathbb{R}$, we have to subtract its mean $\bar{\tau}=\int \tau \mathrm{d} \mu_{0}$, thus we define $\tau_{0}(x)=\tau(x)-\bar{\tau}$. There is a remarkably simple formula for $\bar{\tau}$ that can be obtained by comparing the invariant measures for the billiard map and the billiard flow (see [Che97]):

$$
\bar{\tau}=\frac{\pi(\pi+2 \ell)}{2 \ell+2 \pi} .
$$

On the other hand, we may easily calculate (11) as we have $\tau(r, 0)=2$ whenever $r \in[\pi, \pi+\ell]$ or $r \in[2 \pi+\ell, 2 \pi+2 \ell]$, thus $I_{\tau}=2$ and $I_{\tau_{0}}=2-\frac{\pi(\pi+2 \ell)}{2 \ell+2 \pi}$. This means there is a "best" stadium with $\ell=\ell^{*}=\frac{4 \pi-\pi^{2}}{2 \pi-4} \approx 1.18$ for which $I_{\tau_{0}}=0$ and consequently, by Theorem 1.3 the (centralized) free 
path satisfies the usual central limit theorem. However, whenever $\ell \neq \ell^{*}$, we have $I_{\tau_{0}} \neq 0$ and, by Theorem 1.1] a stronger normalization is needed.

Our interest in $\tau$ is also related to the fact that the billiard flow may be considered as a suspension above the billiard map with the roof function $\tau(x)$. By MT04 suspension flows do inherit some statistical properties from the base transformation, in particular limit theorems, under quite general conditions. Let us denote the billiard flow by

$X_{\tau}=\left\{(x, u) \mid x \in X_{0}, 0 \leq u \leq \tau(x)\right\} / \sim, \quad(x, \tau(x)) \sim\left(T_{0} x, 0\right) \quad S_{t}(x, u)=(x, u+t), \quad \mu_{\tau}=\mu_{0} \times \frac{\text { Leb }}{\bar{\tau}}$

where the action of the flow is understood modulo identifications. Consider a Hölder observable $\Phi: X_{\tau} \rightarrow \mathbb{R}$ satisfying $\int \Phi \mathrm{d} \mu_{\tau}=0$, and define

$$
\Phi_{T}(x)=\int_{0}^{T} \Phi\left(S^{t} x\right) \mathrm{d} t ; \quad J_{\Phi}=\frac{1}{4 \ell}\left[\int_{r \in[\pi, \pi+\ell] \cup[2 \pi+\ell, 2 \pi+2 \ell]} \int_{t \in[0,2]} \Phi(r, 0, t) \mathrm{d} t \mathrm{~d} r\right] .
$$

Corollary 1.4. 1. If $J_{\Phi} \neq 0$, then

$$
\frac{\Phi_{T}}{\sqrt{\frac{c}{\bar{\tau}} T \log T}} \rightarrow \mathcal{N}(0,1) .
$$

Here $c$ is the constant from Theorem 1.1, with I replaced by $J_{\Phi}$.

2. If $J_{\Phi}=0$, then

$$
\frac{\Phi_{T}}{\sqrt{T}} \rightarrow \mathcal{N}\left(0, \sigma_{\Phi}^{2}\right)
$$

for some $\sigma_{\Phi}^{2} \geqslant 0$.

Proof. Define $f_{0}: X_{0} \rightarrow \mathbb{R}$ as $f_{0}(x)=\int_{0}^{\tau(x)} \Phi\left(S_{t}(x, 0)\right) \mathrm{d} t$. Then $f_{0}$ is Hölder, $\int f_{0} \mathrm{~d} \mu_{0}=0$ and $I_{f_{0}}=J_{\Phi}$. Thus, depending on the value of $J_{\Phi}$, one of our two main theorems applies. To show that $\Phi$ inherits the limit behavior from $f_{0}$, we apply the flow version of [Gou03, Theorem A1] recalled as Theorem 5.1] in this paper (see also Remark 5.2). We only need to check that the three conditions of this theorem are satisfied. In case $J_{\Phi} \neq 0$ (and even if $J_{\Phi}=0$ and $\ell=\ell^{*}$ ) conditions 1 and 3 are satisfied with $b=1$. Then condition 2 is merely the Birkhoff ergodic theorem, thus the first statement is established. If $J_{\Phi}=0$, the appropriate normalization for $\tau$ may be $\sqrt{n \log n}$ as opposed to $\sqrt{n}$ needed for $f_{0}$. Thus conditions 1 and 3 of Theorem 5.1 are satisfied for any $0<b<1$, but not for $b=1$. This means condition 2 is to be established for some $b<1$, but this is merely our Remark 5.6. This completes the proof of the second statement.

We will say that a Hölder continuous function $f_{0}: X_{0} \rightarrow \mathbb{R}$ with vanishing integral satisfies $(P 1)$ if $I \neq 0$ and $f_{0}$ vanishes on the set of points $x$ such that $x, T_{0}(x)$ and $T_{0}^{-1}(x)$ belong to the same semicircle, and that $f_{0}$ satisfies $(P 2)$ if $I=0$. We will in fact prove Theorem 1.1 for functions satisfying $(P 1)$, and Theorem 1.3 for functions satisfying $(P 2)$. This will imply Theorem 1.1 in full generality. Namely, if $f_{0}$ is Hölder continuous and satisfies $I \neq 0$, then we may write it as $f_{0}=f_{1}+f_{2}$ where $f_{1}$ satisfies $(P 1)$ and $f_{2}$ satisfies $(P 2)$. By Theorem [1.3, $\frac{S_{n} f_{2}}{\sqrt{n \log n}} \rightarrow 0$. Hence, it is equivalent to have Theorem 1.1 for $f_{0}$ or $f_{1}$. We will comment on the technical reason for introducing the classes (P1) and (P2) in Remark 2.3] below.

In this paper, $C$ will denote a generic constant, that can change from one occurrence to the next. Some constants, which will be used at different places in the paper, will be denoted by $C_{1}, C_{2}, \ldots$ and will have a fixed value. 


\section{Background material on the stadium billiard}

\subsection{Geometric description of the initial map and of an induced map}

The map $T_{0}$ has almost everywhere two nonzero Lyapunov exponent. However, the expansion in the unstable cone (and the contraction in the stable cone) are not uniform: points bouncing many times along the segments, or sliding along the circles, have an expansion arbitrarily close to 1.

To get uniform expansion, we follow Mar04 and [CZ. Let $X$ be the set of points $x$ in $X_{0}$ such that $x$ belongs to a semicircle and $T^{-1}(x)$ does not belong to this semicircle. The set $X_{0}$ is a union of two parallelograms in $X$, and it satisfies

$$
\mu_{0}(X)=\frac{2 \pi}{2(2 \pi+2 \ell)}=\frac{\pi}{2(\pi+\ell)} .
$$

Define a new probability measure on $X$ by

$$
\mathrm{d} \mu=\frac{\cos \theta \mathrm{d} r \mathrm{~d} \theta}{2 \pi}
$$

For $x \in X$, let $\varphi_{+}(x)=\inf \left\{n \geqslant 1, T_{0}^{n}(x) \in X\right\}$. This is the return time of $x$. Let $T: X \rightarrow X$ be the first return map, induced by $T_{0}$ on $X$, i.e., $T(x)=T_{0}^{\varphi_{+}(x)}(x)$. This map preserves the probability measure $\mu$ on $X$. Moreover, it is uniformly hyperbolic in the following sense:

Proposition 2.1. There exists a continuous family of closed cones $\mathcal{C}^{u}(x)$ for $x \in X$, such that $D T(x)\left(\mathcal{C}^{u}(x)\right) \subset \mathcal{C}^{u}(T x)$. Moreover, there exist constants $\Lambda>1$ and $C>0$ such that, for all $x \in X$, for all $v \in \mathcal{C}^{u}(x)$, for all $n \in \mathbb{N}$ such that $T^{n}$ is defined and differentiable at $x$,

$$
\left\|D T^{n}(x) v\right\| \geqslant C \Lambda^{n}\|v\|
$$

Moreover, these cones are uniformly bounded away from the horizontal and vertical directions (i.e., $\{\mathrm{d} \theta=0\}$ and $\{\mathrm{d} r=0\})$.

In the same way, there exist stable cones $\mathcal{C}^{s}(x)$, which satisfy the same properties for $T^{-1}$, except that they are not bounded away from the horizontal direction.

This proposition can be found in [Mar04] and [CZ]. The uniform expansion is given for a different metric, the $p$-metric, satisfying $\|v\|_{p} \leqslant\|v\|$. However, it is easy to check that there exists $C>0$ such that, for all $x \in X$, for all $v \in \mathcal{C}^{u}(x),\|D T(x) v\|_{p} \geqslant C\|v\|$. Hence, the uniform expansion in the $p$-metric implies the same statement for the euclidean metric, up to a constant $C$.

There are two different types of points for which $\varphi_{+}(x)$ can be large: they correspond to points bouncing many times along the segments, or sliding many times along the circles. We will need to describe rather precisely the hyperbolic behavior of $T$ in bouncing regions:

Proposition 2.2. If $x$ is a bouncing point satisfying $\varphi_{+}(x)=n$, then $T$ contracts the p-metric of vectors in the stable cone at least by a factor $\frac{C}{n}$, while $T^{-1}$ contracts the $p$-metric of vectors in the unstable cone at least by a factor $\frac{C}{n}$. Moreover, $T x$ and $T^{-1} x$ are bouncing points with $\varphi_{+}(T x) \geqslant n / 4$, $\varphi_{+}\left(T^{-1} x\right) \geqslant n / 4$ if $n$ is large enough. This implies, in turn, that the above contraction estimates are valid in the euclidean metric as well. 
Remark 2.3. Note that if $x$ is a sliding point satisfying $\varphi_{+}(x)=n$, then we can only guarrantee that $T x$ and $T^{-1} x$ are sliding points with $\varphi_{+}(T x) \geqslant C \sqrt{n}$ and $\varphi_{+}\left(T^{-1} x\right) \geqslant C \sqrt{n}$. This has an unfortunate consequence: we can only apply the coboundary arguments of section 2.3 to functions vanishing along sliding trajectories. Essentially this is the technical reason for introducing the classes (P1) and (P2). The proof of Theorem 1.1 relies heavily on Perron-Frobenius techniques, and thus requires an expanding setting, which implies that collapsing along stable manifolds - coboundary arguments - are essential. Now for the class (P2) it is enough to prove the usual central limit theorem (Theorem 1.3) which can be carried out in a roundabout way in the hyperbolic setting, see section 5.2 .

\section{$2.2 \quad$ Young tower of $T$}

A set $R \subset X$ is a rectangle if there exist $x \in R$ with a local stable manifold $W_{l o c}^{s}(x)$ and a local unstable manifold $W_{l o c}^{u}(x)$, and two Cantor sets $C^{s} \subset W_{l o c}^{s}(x)$ and $C^{u} \subset W_{l o c}^{u}(x)$, such that, for any $y_{s} \in C^{s}$ and $y_{u} \in C^{u}$, then $y_{s}$ has a local unstable manifold $W_{l o c}^{u}\left(y_{s}\right)$ and $y_{u}$ has a local stable manifold $W_{l o c}^{s}\left(y_{u}\right)$. Moreover, these two local manifolds intersect at exactly one point, and this point belongs to $R$.

An $s$-subrectangle of $R$ is a set $\left(\bigcup_{y \in C} W_{l o c}^{s}(y)\right) \cap R$, where $C$ is a subset of $C_{u}$. A $u$-subrectangle is defined in the same way.

[Mar04 and [CZ have proved that $T: X \rightarrow X$ satisfies Chernov's axioms of Che99. This implies that it admits a hyperbolic Young tower in the following sense: there exist a rectangle $R$ of positive measure, a partition $R=\bigcup R_{i}$ (modulo 0) by $s$-subrectangles, and return times $r_{i} \in \mathbb{N}$ such that $T^{r_{i}}$ is a homeomorphism on $R_{i}$, and $T^{r_{i}}\left(R_{i}\right)$ is a $u$-subrectangle of $R$. Moreover, the tails of the tower are exponentially small: there exist $\rho<1$ and $C>0$ such that

$$
\forall n \in \mathbb{N}, \mu\left(\bigcup_{r_{i}>n} R_{i}\right) \leqslant C \rho^{n} .
$$

We can then define an abstract space $\bar{\Delta}$ as the disjoint union of the sets $T^{k}\left(R_{i}\right)$ for $i \in \mathbb{N}$ and $k<r_{i}$. It is endowed with a natural projection $\pi_{X}: \bar{\Delta} \rightarrow X$ and a dynamics $\bar{U}: \bar{\Delta} \rightarrow \bar{\Delta}$ such that $\pi_{X} \circ \bar{U}=T \circ \pi_{X}$.

It is also possible to construct on $\bar{\Delta}$ a probability measure $\mu_{\bar{\Delta}}$ which is invariant under $\bar{U}$ and such that $\left(\pi_{X}\right)_{*}\left(\mu_{\bar{\Delta}}\right)=\mu$. Note however that $\pi_{X}$ is in general strongly not injective, so that $\mu_{\bar{\Delta}}$ can not be defined as the pullback of $\mu$. Rather, one constructs an invariant measure for $\bar{U}$, and one proves that its projection, being absolutely continuous with respect to $\mu$ and $T$-invariant, is necessarily $\mu$.

It is then useful to go from this abstract hyperbolic dynamics to an abstract expanding dynamics. To do so, one identifies the points of $\bar{\Delta}$ which are on the same stable leaf in some rectangle. This defines a space $\Delta$, together with a projection $\pi_{\Delta}: \bar{\Delta} \rightarrow \Delta$. Since the map $\bar{U}$ sends stable leaves to stable leaves, it gives rise to a dynamics $U: \Delta \rightarrow \Delta$ on the quotient. The measure $\mu_{\Delta}:=\left(\pi_{\Delta}\right)_{*}\left(\mu_{\bar{\Delta}}\right)$ is invariant under $U$. Then $\left(\Delta, U, \mu_{\Delta}\right)$ is an expanding Young tower, in the sense of Section 3.2

\subsection{Coboundary results}

Let $f_{0}: X_{0} \rightarrow \mathbb{R}$ be a Hölder function satisfying $(P 1)$, for which we want to prove a limit theorem. Since it is easier to work in an expanding and well understood setting, we will first prove results in 
$\Delta$, and then go back from $\Delta$ to $X_{0}$.

For $x \in X$, let first $f(x)=\sum_{k=0}^{\varphi_{+}(x)-1} f_{0}\left(T_{0}^{k} x\right)$. This function is not bounded any more. However, if two points $x$ and $y$ are on a local stable manifold which is not cut by a discontinuity of $T_{0}$ during the next $n$ iterates of $T_{0}$, and with $\varphi_{+}(x)=\varphi_{+}(y)=n$, then

$$
|f(x)-f(y)| \leqslant C n d(x, y)^{\alpha}
$$

for some $\alpha>0$. In the same way, if $x$ and $y$ are on a local unstable manifold which is not cut during the next $n$ iterates of $T_{0}$, and $\varphi_{+}(x)=\varphi_{+}(y)=n$, then $|f(x)-f(y)| \leqslant C n d(T x, T y)^{\alpha}$. Moreover, the property $(P 1)$ implies that $f$ is bounded on the set of points sliding along the semicircles.

The measure of points bouncing $n$ times along the segments is $\sim \frac{\ell^{2}}{\pi n^{3}}$. Since $I$ (given by (II)) is nonzero, the function $f$ is equivalent to $n I$ on this set, and we obtain

$$
\mu\{x|| f(x) \mid \geqslant n\} \sim \sum_{n /|I|}^{\infty} \frac{\ell^{2}}{\pi k^{3}} \sim \frac{I^{2} l^{2}}{2 \pi n^{2}} .
$$

Hence, the distribution of $f$ is in the nonstandard domain of attraction of the Gaussian law (see Paragraph 3.11).

Define a function $\bar{f}$ on $\bar{\Delta}$ by $\bar{f}=f \circ \pi_{X}$. It would be easy to go finally from $\bar{\Delta}$ to $\Delta$ if $\bar{f}$ were constant along the local stable leaves in $\bar{\Delta}$ (which would mean that $\bar{f}$ would induce a function on the quotient $\Delta$ ). This is in general not the case, but we will prove that $\bar{f}$ is cohomologous to such a function, using the usual cohomology trick.

For every rectangle in $\bar{\Delta}$, choose a definite unstable leaf. Define a projection $\pi: \bar{\Delta} \rightarrow \bar{\Delta}$ by sliding along stable manifolds to this specific unstable manifold. We define a function $\bar{u}(x)=\sum_{k=0}^{\infty}\left[\bar{f}\left(\bar{U}^{k} x\right)-\right.$ $\left.\bar{f}\left(\bar{U}^{k} \pi x\right)\right]$. Note that, despite of the fact that $T$ contracts stable manifolds uniformly, the function $\bar{u}(x)$ may not seem well-defined at first sight, as $\bar{f}$ - and consequently, its Hölder constant - is unbounded. Nevertheless, whenever $\bar{f}$ is large, $T$ contracts stable manifolds strongly, and the Hölder constant can be regained by going down the tower. This is the essence of the following lemma.

Lemma 2.4. The function $\bar{u}$ is well defined and bounded on $\bar{\Delta}$.

Proof. In this proof the positive constants $C$ do depend on the Hölder exponent $\alpha$, but this has no significance. Let $K \in \mathbb{N}$ be such that $\alpha K>1$. Consider first $x$ which is at height $\geqslant K$ in the tower. Let $y=\pi x$. Let $x^{\prime}=\bar{U}^{-K} x$ and $y^{\prime}=\bar{U}^{-K} y$. We will prove that

$$
\forall k \in \mathbb{N},\left|\bar{f}\left(\bar{U}^{k} x\right)-\bar{f}\left(\bar{U}^{k} y\right)\right| \leqslant C d\left(\pi_{X} \bar{U}^{k} x^{\prime}, \pi_{X} \bar{U}^{k} y^{\prime}\right)^{\alpha} .
$$

Namely, if $\varphi_{+}\left(\pi_{X} \bar{U}^{k} x\right)=n$, then

$$
\left|\bar{f}\left(\bar{U}^{k} x\right)-\bar{f}\left(\bar{U}^{k} y\right)\right| \leqslant C n d\left(\pi_{X} \bar{U}^{k} x, \pi_{X} \bar{U}^{k} y\right)^{\alpha}=C n d\left(\pi_{X} \bar{U}^{k+K} x^{\prime}, \pi_{X} \bar{U}^{k+K} y^{\prime}\right)^{\alpha}
$$

by (4). If $n=\varphi_{+}\left(\pi_{X} \bar{U}^{k} x\right)$ is bounded, the conclusion is trivial. If $n$ is large and $\pi_{X} \bar{U}^{k} x$ is a sliding point, the conclusion is also trivial by $(P 1)$.

Hence, assume that $n$ is large and that $\pi_{X} \bar{U}^{k} x$ is a bouncing point. Proposition 2.2 implies that, for $0 \leqslant i<K, \varphi_{+}\left(\pi_{X} \bar{U}^{k+i} x^{\prime}\right) \geqslant n / 4^{K-i} \geqslant n / 4^{K}$. Once again by Proposition 2.2, we get

$$
d\left(\pi_{X} \bar{U}^{k+i+1} x^{\prime}, \pi_{X} \bar{U}^{k+i+1} y^{\prime}\right) \leqslant \frac{C}{n} d\left(\pi_{X} \bar{U}^{k+i} x^{\prime}, \pi_{X} \bar{U}^{k+i} y^{\prime}\right) .
$$


Hence,

$$
d\left(\pi_{X} \bar{U}^{k+K} x^{\prime}, \pi_{X} \bar{U}^{k+K} y^{\prime}\right) \leqslant \frac{C}{n^{K}} d\left(\pi_{X} \bar{U}^{k} x^{\prime}, \pi_{X} \bar{U}^{k} y^{\prime}\right) .
$$

Together with (77) and the inequality $K \alpha>1$, this implies (6).

Since $\pi_{X} x^{\prime}$ and $\pi_{X} y^{\prime}$ are on a local stable manifold, $d\left(\pi_{X} \bar{U}^{k} x^{\prime}, \pi_{X} \bar{U}^{k} y^{\prime}\right)$ goes exponentially fast to zero. Hence, the series $\sum\left|\bar{f}\left(\bar{U}^{k} x\right)-\bar{f}\left(\bar{U}^{k} y\right)\right|$ is summable, and $\bar{u}(x)$ is well defined.

Suppose now that $x$ is at height $<K$. Let $y=\pi x$. Applying the previous argument to $x^{\prime}=x$ and $y^{\prime}=y$, we get that $\sum_{k=K}^{\infty}\left|\bar{f}\left(\bar{U}^{k} x\right)-\bar{f}\left(\bar{U}^{k} y\right)\right|$ is bounded. Moreover, during the first $K$ iterates, $x$ and $y$ remain at a bounded height in the tower, which implies that $\bar{f}\left(\bar{U}^{k} x\right)$ and $\bar{f}\left(\bar{U}^{k} y\right)$ remain uniformly bounded. This concludes the proof.

Let $\bar{g}(x)=\bar{f}(x)-\bar{u}(x)+\bar{u}(\bar{U} x)$. Then

$$
\bar{g}(x)=\bar{f}(\pi x)+\sum_{k=0}^{\infty}\left[\bar{f}\left(\bar{U}^{k}(\bar{U} \pi x)\right)-\bar{f}\left(\bar{U}^{k}(\pi \bar{U} \pi x)\right)\right]
$$

Hence, $\bar{g}(x)$ depends only on $\pi x$, i.e., $\bar{g}$ is constant along the stable manifolds in the rectangles. Consequently, there exists a function $g: \Delta \rightarrow \mathbb{R}$ such that $\bar{g}=g \circ \pi_{\Delta}$.

It will be important that $g$ is regular enough on $\Delta$, to use functional analytic techniques. For $x_{1}, x_{2} \in \Delta$, let $s\left(x_{1}, x_{2}\right)$ be their separation time, i.e., the number of returns to the basis before $x_{1}$ and $x_{2}$ get into different elements of the partition. To obtain the following lemma, we will use several times the same argument as in Lemma 2.4, but sometimes along unstable manifolds instead of stable ones.

Lemma 2.5. There exist $C>0$ and $\tau<1$ such that, for every $x_{1}, x_{2}$ in the same element of partition of $\Delta$,

$$
\left|g\left(x_{1}\right)-g\left(x_{2}\right)\right| \leqslant C \tau^{s\left(x_{1}, x_{2}\right)}
$$

Proof. Let us first prove that, if $x_{1}, x_{2}$ belong to the same unstable leaf in a rectangle of $\bar{\Delta}$, then

$$
\bar{g}\left(x_{1}\right)-\bar{g}\left(x_{2}\right) \text { is uniformly bounded. }
$$

The same argument as in the proof of Lemma 2.4 shows that $\sum_{k=0}^{\infty}\left[\bar{f}\left(\bar{U}^{k}(\bar{U} \pi x)\right)-\bar{f}\left(\bar{U}^{k}(\pi \bar{U} \pi x)\right)\right]$ is bounded. Hence, it is sufficient to prove that $\bar{f}\left(\pi x_{1}\right)-\bar{f}\left(\pi x_{2}\right)$ is bounded. Let $K$ be as in the proof of Lemma 2.4 If $x_{1}$ (and $\left.x_{2}\right)$ return to the basis of $\bar{\Delta}$ before time $K$, then $\varphi_{+}\left(x_{1}\right)=\varphi_{+}\left(x_{2}\right)$ is bounded, which implies that $\bar{f}\left(\pi x_{1}\right)$ and $\bar{f}\left(\pi x_{2}\right)$ are bounded. If $x_{1}$ (and $\left.x_{2}\right)$ are sliding points, then the conclusion is also a consequence of $(P 1)$. Otherwise, $x_{1}$ and $x_{2}$ are bouncing points. We show as in the proof of Lemma 2.4 (but along the unstable leaf containing $\pi x_{1}$ and $\pi x_{2}$ ) that $\left|\bar{f}\left(\pi x_{1}\right)-\bar{f}\left(\pi x_{2}\right)\right| \leqslant C d\left(\pi_{X} \bar{U}^{K} \pi x_{1}, \pi_{X} \bar{U}^{K} \pi x_{2}\right)^{\alpha}$. Since this quantity is uniformly bounded, this concludes the proof of (8) .

Take $x_{1}, x_{2} \in \bar{\Delta}$ on the same unstable leaf, and let $s=s\left(\pi_{\Delta}\left(x_{1}\right), \pi_{\Delta}\left(x_{2}\right)\right)$. We will prove that

$$
\left|\bar{g}\left(x_{1}\right)-\bar{g}\left(x_{2}\right)\right| \leqslant C \lambda^{\alpha s / 2}
$$

for some $C>0$, where $\lambda<1$ is larger than the contraction coefficient of $T$ along stable manifolds, and the contraction coefficient of $T^{-1}$ along unstable manifolds. 
By (8), this is trivial if $s<2 K$. Hence, we can assume $s \geqslant 2 K$. Let $N=\left\lfloor\frac{s}{2}\right\rfloor \geqslant K$, then

$$
\begin{aligned}
\bar{g}\left(x_{1}\right)-\bar{g}\left(x_{2}\right) & =\bar{f}\left(\pi x_{1}\right)-\bar{f}\left(\pi x_{2}\right) \\
& +\sum_{k=0}^{N-1}\left[\bar{f}\left(\bar{U}^{k}\left(\bar{U} \pi x_{1}\right)\right)-\bar{f}\left(\bar{U}^{k}\left(\bar{U} \pi x_{2}\right)\right)\right]+\sum_{k=0}^{N-1}\left[\bar{f}\left(\bar{U}^{k}\left(\pi \bar{U} \pi x_{2}\right)\right)-\bar{f}\left(\bar{U}^{k}\left(\pi \bar{U} \pi x_{1}\right)\right)\right] \\
& +\sum_{k=N}^{\infty}\left[\bar{f}\left(\bar{U}^{k}\left(\bar{U} \pi x_{1}\right)\right)-\bar{f}\left(\bar{U}^{k}\left(\pi \bar{U} \pi x_{1}\right)\right)\right]+\sum_{k=N}^{\infty}\left[\bar{f}\left(\bar{U}^{k}\left(\pi \bar{U} \pi x_{2}\right)\right)-\bar{f}\left(\bar{U}^{k}\left(\bar{U} \pi x_{2}\right)\right)\right] .
\end{aligned}
$$

Since $N+K \leqslant s$, we have for any $k<N$

$$
\begin{aligned}
\left|\bar{f}\left(\bar{U}^{k}\left(\bar{U} \pi x_{1}\right)\right)-\bar{f}\left(\bar{U}^{k}\left(\bar{U} \pi x_{2}\right)\right)\right| & \leqslant C d\left(\pi_{X} \bar{U}^{k+K}\left(\bar{U} \pi x_{1}\right), \pi_{X} \bar{U}^{k+K}\left(\bar{U} \pi x_{2}\right)\right)^{\alpha} \\
& \leqslant C \lambda^{\alpha(s-(k+K+1))} d\left(\pi_{X} \bar{U}^{s} \pi x_{1}, \pi_{X} \bar{U}^{s} \pi x_{2}\right)^{\alpha} \leqslant C \lambda^{\alpha(s-k)} .
\end{aligned}
$$

Summing over $k$, we obtain

$$
\left|\sum_{k=0}^{N-1}\left[\bar{f}\left(\bar{U}^{k}\left(\bar{U} \pi x_{1}\right)\right)-\bar{f}\left(\bar{U}^{k}\left(\bar{U} \pi x_{2}\right)\right)\right]\right| \leqslant C \lambda^{\alpha(s-N)} \leqslant C \lambda^{\alpha s / 2} .
$$

The other term on the second line of (10) can be estimated in the same way, as well as the term on the first line of (10).

Since $N \geqslant K$, we also have for any $k \geqslant N$

$$
\begin{aligned}
\left|\bar{f}\left(\bar{U}^{k}\left(\bar{U} \pi x_{1}\right)\right)-\bar{f}\left(\bar{U}^{k}\left(\pi \bar{U} \pi x_{1}\right)\right)\right| & \leqslant C d\left(\pi_{X} \bar{U}^{k-K}\left(\bar{U} \pi x_{1}\right), \pi_{X} \bar{U}^{k-K}\left(\pi \bar{U} \pi x_{1}\right)\right)^{\alpha} \\
& \leqslant C \lambda^{\alpha(k-K)} d\left(\pi_{X}\left(\bar{U} \pi x_{1}\right), \pi_{X}\left(\pi \bar{U} \pi x_{1}\right)\right)^{\alpha} \leqslant C \lambda^{\alpha k}
\end{aligned}
$$

Summing over $k$, we obtain

$$
\left|\sum_{k=N}^{\infty}\left[\bar{f}\left(\bar{U}^{k}\left(\bar{U} \pi x_{1}\right)\right)-\bar{f}\left(\bar{U}^{k}\left(\pi \bar{U} \pi x_{1}\right)\right)\right]\right| \leqslant C \lambda^{\alpha N} \leqslant C \lambda^{\alpha s / 2} .
$$

The other term on the third line of (10) is handled in the same way.

\section{Limit theorems in Young towers}

\subsection{A result by Aaronson and Denker}

A function $f: \mathbb{R}_{+}^{*} \rightarrow \mathbb{R}_{+}^{*}$ is slowly varying if, for all $\lambda>0, f(\lambda x) / f(x)$ tends to 1 when $x \rightarrow \infty$.

By classical probabilistic results, a real random variable $Z$ is in the nonstandard domain of attraction of the Gaussian distribution $\mathcal{N}(0,1)$ if and only if it satisfies one of the following equivalent conditions:

- The function $L(x):=E\left(Z^{2} 1_{|Z| \leqslant x}\right)$ is unbounded and slowly varying.

- $P(|Z|>x) \sim x^{-2} l(x)$ for some function $l$ such that $\widetilde{L}(x):=2 \int_{1}^{x} \frac{l(u)}{u} \mathrm{~d} u$ is unbounded and slowly varying. 
Remark 3.1. In this case, $\widetilde{L}(x) \sim L(x)$ when $x \rightarrow \infty$, and $l(x)=o(L(x))$. It is possible, however, that $l$ is not slowly varying and that these conditions hold anyway.

Such a random variable belongs to $L^{p}$ for all $1 \leqslant p<2$, but not to $L^{2}$. We will say that $l$ and $L$ are the tail functions of $Z$. They are defined up to asymptotic equivalence. Choose a sequence $B_{n} \rightarrow \infty$ such that $\frac{n}{B_{n}^{2}} L\left(B_{n}\right) \rightarrow 1$. Then, if $Z_{0}, Z_{1}, \ldots$ is a sequence of independent random variables distributed as $Z$, then

$$
\frac{Z_{0}+\cdots+Z_{n-1}-n E(Z)}{B_{n}} \rightarrow \mathcal{N}(0,1)
$$

More generally, if $\frac{n}{B_{n}^{2}} L\left(B_{n}\right) \rightarrow C>0$, then the previous sequence converges to $\mathcal{N}(0, C)$.

In [AD01], Aaronson and Denker have proved the same kind of limit theorem when the sequence $Z_{0}, Z_{1}, \ldots$ is not independent. More precisely, consider $U$ a mixing Gibbs-Markov map (as defined in Aar97) on a space $\Delta$, preserving a probability measure $\mu_{\Delta}$, and let $g: \Delta \rightarrow \mathbb{R}$ be a function which is locally Hölder and whose distribution with respect to $\mu_{\Delta}$ is in the nonstandard domain of attraction of $\mathcal{N}(0,1)$ as above. Then they prove that

$$
\frac{g+g \circ U+\cdots+g \circ U^{n-1}-n \int g}{B_{n}} \rightarrow \mathcal{N}(0,1)
$$

as above.

The proof goes as follows: let $\widehat{U}$ be the transfer operator associated to $U$, and $\widehat{U}_{t}$ its perturbation given by $\widehat{U}_{t} u=\widehat{U}\left(e^{i t g} u\right)$. These operators satisfy a Lasota-Yorke inequality on the space of Hölder functions, and $\left\|\widehat{U}_{t}-\widehat{U}\right\|=O(t)$. Hence, the eigenvalue $\lambda_{t}$ of $\widehat{U}_{t}$ close to 1 satisfies $\left|\lambda_{t}-1\right|=O(t)$, and the corresponding eigenfunction $w_{t}$ (normalized so that $\int w_{t}=1$ ) is such that $\left\|w_{t}-1\right\|=O(t)$.

Then they prove the following abstract lemma (in which there is no dynamics, this lemma depends only on the distribution of $g$ ):

Lemma 3.2. For any bounded function $w$ on $\Delta$,

$$
\int\left(e^{i t g}-1-i t g\right) w=-\frac{t^{2}}{2} \int 1_{|g| \leqslant 1 /|t|} g^{2} w+\|w\|_{\infty} o\left(t^{2} L(1 /|t|)\right) .
$$

Here, the o $\left(t^{2} L(1 /|t|)\right)$ is uniform in $w$.

Applying this lemma to $w_{t}$, one gets

$$
\lambda_{t}-1-i t \int g w_{t}=\int\left(e^{i t g}-1-i t g\right) w_{t}=-\frac{t^{2}}{2} \int 1_{|g| \leqslant 1 /|t|} g^{2} w_{t}+o\left(t^{2} L(1 /|t|)\right)
$$

(where we have used the fact that $w_{t}$ is bounded). Since $\left\|w_{t}-1\right\|_{\infty}=o(1)$,

$$
\frac{t^{2}}{2} \int 1_{|g| \leqslant 1 /|t|} g^{2} w_{t}=\frac{t^{2}}{2} L(1 /|t|)(1+o(1)) .
$$

Hence,

$$
\lambda_{t}=1+i t \int g w_{t}-\frac{t^{2}}{2} L(1 /|t|)(1+o(1)) .
$$

Finally, $\int g w_{t}=\int g+O(t)$ since $\left\|w_{t}-1\right\|_{\infty}=O(t)$. So we get

$$
\lambda_{t}=1+i t \int g-\frac{t^{2}}{2} L(1 /|t|)(1+o(1)) .
$$

This expansion is sufficient to get the required limit theorem. 


\subsection{The result in Young towers}

Let $\left(\Delta, \mu_{\Delta}\right)$ be a probability space and $U: \Delta \rightarrow \Delta$ a probability preserving map. We say that $(\Delta, U)$ is an expanding Young tower ([You99]) if there exist integers $r_{p} \in \mathbb{N}^{*}$ and a partition $\left\{\Delta_{k, p}\right\}_{p \in \mathbb{N}, k \in\left\{0, \ldots, r_{p}-1\right\}}$ of $\Delta$ such that

1. For all $p$ and $k<r_{p}-1, T$ is a measurable isomorphism between $\Delta_{k, p}$ and $\Delta_{k+1, p}$, preserving $\mu_{\Delta}$.

2. For all $p, T$ is a measurable isomorphism between $\Delta_{r_{p}-1, p}$ and $\Delta_{0}:=\bigcup_{m} \Delta_{0, m}$.

3. Let $U_{0}$ be the first return map induced by $U$ on $\Delta_{0}$. For $x, y \in \Delta_{0}$, define their separation time $s(x, y)=\inf \left\{n \in \mathbb{N} \mid U_{0}^{n}(x)\right.$ and $U_{0}^{n}(y)$ are not in the same $\left.\Delta_{0, p}\right\}$. We extend this separation time to the whole tower in the following way: if $x, y$ are not in the same element of partition, set $s(x, y)=0$. Otherwise, $x, y \in \Delta_{k, p}$. Let $x^{\prime}, y^{\prime} \in \Delta_{0, p}$ be such that $x=U^{k} x^{\prime}$ and $y=U^{k} y^{\prime}$, and set $s(x, y)=s\left(x^{\prime}, y^{\prime}\right)$.

For $x \in \Delta$, let $J(x)$ be the inverse of the jacobian of $U$ at $x$. We assume that there exist $\beta<1$ and $C>0$ such that, for all $x, y$ in the same element of partition,

$$
\left|1-\frac{J(x)}{J(y)}\right| \leqslant C \beta^{s(U x, U y)}
$$

Remark 3.3. Note that the definition of separation time in You98 is in terms of the number of all iterations of $U$, while we follow the convention of [You99] when we define separation in terms of returns to the basis. Hence, our setting is more general than that of [You98, but it will make the proof of the spectral gap more complicated.

Let $\Delta_{n}=\bigcup \Delta_{n, p}$. This is the set of points at height $n$ in the tower. We will say that $(U, \Delta)$ is an expanding Young tower with exponentially small tail if there exists $\rho<1$ such that $\mu_{\Delta}\left(\Delta_{n}\right)=O\left(\rho^{n}\right)$.

Let $J^{(n)}$ be the inverse of the jacobian of $U^{n}$. It is standard that (12) implies that the distortion of the iterates of $U$ is uniformly bounded, in the following sense: there exists $C>0$ such that, for all points $x, y$ such that $T^{k}$ and $T^{k} y$ remain in the same elements of the partition for $0 \leqslant k<n$,

$$
\left|1-\frac{J^{(n)}(x)}{J^{(n)}(y)}\right| \leqslant C \beta^{s\left(U^{n} x, U^{n} y\right)} .
$$

A function $g: \Delta \rightarrow \mathbb{R}$ is locally Hölder if there exist $C>0$ and $\tau<1$ such that $|g(x)-g(y)| \leqslant C \tau^{s(x, y)}$ for all $x, y$ in the same element of the partition. This is exactly the type of functions that arise from the stadium billiard, cf. Lemma 2.5. Note that $g$ can very well be unbounded. Without loss of generality, we can assume $\tau \geqslant \beta$.

Let $\omega(x)$ be the height of the point $x$, i.e., $\omega(x)=n$ if $x \in \Delta_{n}$. Let $\pi_{0}: \Delta \rightarrow \Delta_{0}$ be the projection to the basis, and define a function $G$ on $\Delta$ by $G(x)=\sum_{k=0}^{\omega(x)-1} g\left(U^{k} \pi_{0} x\right)$. In this setting, we get the following extension of the theorem proved by Aaronson and Denker:

Theorem 3.4. Let $U: \Delta \rightarrow \Delta$ be an expanding Young tower with exponentially small tail, and let $g: \Delta \rightarrow \mathbb{R}$ be locally Hölder continuous. Assume that the distribution of $g$ is in the nonstandard domain of attraction of $\mathcal{N}(0,1)$, with tail functions $l$ and $L$. Assume moreover that $l$ and $L$ are 
slowly varying, and $l(x \ln x) / l(x) \rightarrow 1, L(x \ln x) / L(x) \rightarrow 1$ when $x \rightarrow \infty$. Finally, assume that there exists a real number $a \neq-1 / 2$ such that

$$
\left.\int g\left(e^{i t G}-1\right)=(a+o(1)) i t L(1 /|t|)\right) \text { when } t \rightarrow 0 .
$$

Write $L_{1}(x)=(2 a+1) L(x)$, and choose a sequence $B_{n} \rightarrow \infty$ such that $\frac{n}{B_{n}^{2}} L_{1}\left(B_{n}\right) \rightarrow 1$. Then

$$
\frac{S_{n} g-n \int g}{B_{n}} \rightarrow \mathcal{N}(0,1)
$$

The additional assumption on $l$ and $L$ is satisfied in most natural cases (for example when $l=1$ and $L=\ln$, which will be the case for the stadium billiard).

When $a=0$, we get the same asymptotics as in Aaronson-Denker's Theorem. However, when $a \neq 0$, then there is an additional effect due to the presence of the tower.

The proof will follow the same lines as in Aaronson-Denker's proof: it is possible to construct a good space on which the transfer operator $\widehat{U}$ has a spectral gap. The perturbed operator $\widehat{U}_{t}$ also has a spectral gap, which gives an eigenvalue $\lambda_{t}$ and an eigenfunction $w_{t}$. The main problem is that $\left\|\widehat{U}_{t}-\widehat{U}\right\|$ can not be $O(t)$ in general: it is easy to construct examples where $t=o\left(\left\|\left(\widehat{U}_{t}-\widehat{U}\right) 1\right\|_{L^{2}}\right)$, whence $t=o\left(\left\|\widehat{U}_{t}-\widehat{U}\right\|\right)$ as soon as the good space is contained in $L^{2}$ and contains the function 1 .

Using abstract arguments by Keller and Liverani, we can nevertheless prove that $\left|\lambda_{t}-1\right|=O\left(|t|^{1 / 10}\right)$ and $\left\|w_{t}-1\right\|_{L^{1}}=O\left(|t|^{1 / 10}\right)$. These information are (essentially) sufficient to apply Aaronson and Denker's argument and get $\lambda_{t}=1+i t \int g w_{t}-\frac{t^{2}}{2} \int 1_{|g| \leqslant 1 /|t|} g^{2} w_{t}+o\left(t^{2} L(1 /|t|)\right)$ as in (11). The main difficulty is then to make the function $w_{t}$ disappear in this expression, to get something more tractable. We will namely show that $\int 1_{|g| \leqslant 1 /|t|} g^{2} w_{t} \sim L(1 /|t|)$ and $\int g w_{t} \sim \int g e^{i t G}$, which will conclude the proof.

To do this, we need to know that $w_{t}-1=O(t)$ in some sense. To prove such an estimate, we use a roundabout technical argument relying on the fact that the induced map on the basis of the tower is uniformly expanding, to prove that $\left\|1_{\Delta_{0}}\left(w_{t}-1\right)\right\|_{\infty}=O(t)$, and then we propagate this information up in the tower, using the information we have already proved on $\lambda_{t}$. This propagation requires the Birkhoff sums of $g$ to be small enough. To ensure this on a set of large measure, we use the information on the tails of $g$. This is the only point where the additional information on $l$ and $L$ is used.

\subsection{Proof of Theorem 3.4}

We will first prove Theorem 3.4 assuming that $\int g=0$. In Paragraph 3.3.5 we will show that this implies the theorem in full generality. Hence, until the end of Paragraph 3.3.4, we will assume that $\int g=0$.

\subsubsection{Construction of the functional spaces and the transfer operators}

Since the tails of the tower are exponentially small by assumption, there exists $\rho<1$ such that $\mu_{\Delta}\left(\Delta_{n}\right) \leqslant C \rho^{n}$. Denote the return time to the basis from itself by $\varphi$. Take $\varepsilon>0$ such that $e^{6 \varepsilon} \rho<1$. 
For $u: \Delta \rightarrow \mathbb{C}$, write

$$
\|u\|_{m}=\inf \left\{C \mid \forall n \in \mathbb{N} \text {, for almost every } x \in \Delta_{n},|u(x)| \leqslant C e^{\varepsilon n}\right\}
$$

and

$\|u\|_{l}=\inf \{C \mid$ for almost every $x, y$ in the same element of the partition at height $n$,

$$
\left.|u(x)-u(y)| \leqslant C e^{\varepsilon n} \tau^{s(x, y)}\right\} .
$$

Denote by $\mathcal{H}$ the space of measurable functions $u$ on $\Delta$ for which $\|u\|:=\|u\|_{m}+\|u\|_{l}<+\infty$. It is a Banach space included in $L^{1}$ (and even in $L^{6}$ because of the condition $e^{6 \varepsilon} \rho<1$ ). This inclusion is compact.

The following proposition is similar to a result of Young:

Proposition 3.5. There exist $C>0$ and $\theta<1$ such that, for any $u \in \mathcal{H}$, for any $n \in \mathbb{N}$,

$$
\left\|\widehat{U}^{n} u\right\| \leqslant C \theta^{n}\|u\|+C\|u\|_{L^{1}} .
$$

Note that our definition of separation time is not the same as in You98, and that Young uses the fact that the return to the basis only occur after a large time $N$. This gives her a strong expansion, sufficient to get rid of constants easily. This is not true in our setting. Hence, the proof of the proposition will be more involved than Young's.

Proof. Take $x \in \Delta_{0}$. Then $\widehat{U}^{n} u(x)=\sum J^{(n)}\left(x_{p}\right) u\left(x_{p}\right)$, where $\left\{x_{p}\right\}=U^{-n}(x)$. Let $A_{p}$ containing $x_{p}$ be such that $U^{n}: A_{p} \rightarrow \Delta_{0}$ is an isomorphism. Then $J^{(n)}\left(x_{p}\right) \leqslant C \mu_{\Delta}\left(A_{p}\right)$ since the distortion is bounded, by (13). Let $\omega_{p}$ be the height of the set $A_{p}$ and $r_{p}$ the number of returns of $A_{p}$ to the basis before time $n$.

For $y \in A_{p}, s\left(x_{p}, y\right) \geqslant r_{p}$, whence

$$
\left|u\left(x_{p}\right)-u(y)\right| \leqslant \tau^{r_{p}} e^{\varepsilon \omega_{p}}\|u\|_{l}
$$

Hence,

$$
\left|u\left(x_{p}\right)\right| \leqslant \tau^{r_{p}} e^{\varepsilon \omega_{p}}\|u\|_{l}+\frac{1}{\mu_{\Delta}\left(A_{p}\right)} \int_{A_{p}}|u| .
$$

We get

$$
\left|\widehat{U}^{n} u(x)\right| \leqslant C \sum \mu_{\Delta}\left(A_{p}\right) \tau^{r_{p}} e^{\varepsilon \omega_{p}}\|u\|_{l}+C \int|u| .
$$

Let $\omega: \Delta \rightarrow \mathbb{N}$ be the function "height", and let $\Psi_{n}(x)$ be the number of returns of $x$ to the basis between time 1 and $n$. Then (16) implies that

$$
\left|\widehat{U}^{n} u(x)\right| \leqslant C\|u\|_{l} \int_{U^{-n} \Delta_{0}} \tau^{\Psi_{n}} e^{\varepsilon \omega}+C\|u\|_{L^{1}} .
$$

We will use the following technical lemma, which will be proved in the appendix.

Lemma 3.6. There exist $C>0$ and $\theta<1$ such that, for any $n \in \mathbb{N}$,

$$
\int_{U^{-n} \Delta_{0}} \tau^{\Psi_{n}} e^{\varepsilon \omega} \leqslant C \theta^{n}
$$


Increasing $\theta$ if necessary, we can assume that $e^{-\varepsilon} \leqslant \theta$.

This lemma, together with (17), implies that, for any $x \in \Delta_{0}$,

$$
\left|\widehat{U}^{n} u(x)\right| \leqslant C \theta^{n}\|u\|_{l}+C\|u\|_{L^{1}}
$$

Consider now $x \in \Delta$ such that $\omega(x)=k<n$. Let $x^{\prime}$ be its projection in the basis. Then $\widehat{U}^{n} u(x)=$ $\widehat{U}^{n-k} u\left(x^{\prime}\right)$, whence

$$
e^{-\varepsilon k}\left|\widehat{U}^{n} u(x)\right|=e^{-\varepsilon k}\left|\widehat{U}^{n-k} u\left(x^{\prime}\right)\right| \leqslant e^{-\varepsilon k} C \theta^{n-k}\|u\|_{l}+C e^{-\varepsilon k}\|u\|_{L^{1}} \leqslant C \theta^{n}\|u\|_{l}+C\|u\|_{L^{1}} .
$$

Assume finally that $\omega(x)=k \geqslant n$. Let $x^{\prime}=U^{-n}(x)$. Then

$$
e^{-\varepsilon k}\left|\widehat{U}^{n} u(x)\right|=e^{-\varepsilon n} e^{-\varepsilon(k-n)}\left|u\left(x^{\prime}\right)\right| \leqslant e^{-\varepsilon n}\|u\|_{m} .
$$

These equations prove that

$$
\left\|\widehat{U}^{n} u\right\|_{m} \leqslant C \theta^{n}\|u\|+C\|u\|_{L^{1}}
$$

We still have to handle the Hölder norm. Consider two points $x, y$ in the same element of partition of the basis $\Delta_{0}$. Let $x_{p}$ and $y_{p}$ be their preimages, in sets $A_{p}$ as above. Then

$$
\begin{aligned}
\left|\widehat{U}^{n} u(x)-\widehat{U}^{n} u(y)\right| & \leqslant \sum\left|J^{(n)}\left(x_{p}\right) u\left(x_{p}\right)-J^{(n)}\left(y_{p}\right) u\left(y_{p}\right)\right| \\
& \leqslant \sum\left|J^{(n)}\left(x_{p}\right)\right|\left|u\left(x_{p}\right)-u\left(y_{p}\right)\right|+\sum\left|J^{(n)}\left(x_{p}\right)\right|\left|1-\frac{J^{(n)}\left(y_{p}\right)}{J^{(n)}\left(x_{p}\right)}\right|\left|u\left(y_{p}\right)\right| .
\end{aligned}
$$

In the first sum, $\left|J^{(n)}\left(x_{p}\right)\right| \leqslant C \mu_{\Delta}\left(A_{p}\right)$ and $\left|u\left(x_{p}\right)-u\left(y_{p}\right)\right| \leqslant \tau^{s(x, y)+r_{p}} e^{\varepsilon \omega_{p}}\|u\|_{l}$. Hence,

$$
\sum\left|J^{(n)}\left(x_{p}\right)\right|\left|u\left(x_{p}\right)-u\left(y_{p}\right)\right| \leqslant C \tau^{s(x, y)}\|u\|_{l} \int_{U^{-n} \Delta_{0}} \tau^{\Psi_{n}} e^{\varepsilon \omega} \leqslant C \theta^{n} \tau^{s(x, y)}\|u\|_{l}
$$

by Lemma 3.6.

In the second sum, $\left|J^{(n)}\left(x_{p}\right)\right| \leqslant C \mu_{\Delta}\left(A_{p}\right)$ and $\left|1-\frac{J^{(n)}\left(y_{p}\right)}{J^{(n)}\left(x_{p}\right)}\right| \leqslant C \tau^{s(x, y)}$ by (13). Moreover, $\left|u\left(y_{p}\right)\right|$ is bounded by (15). Using these inequalities, we get

$$
\begin{aligned}
\sum\left|J^{(n)}\left(x_{p}\right)\right|\left|1-\frac{J^{(n)}\left(y_{p}\right)}{J^{(n)}\left(x_{p}\right)}\right|\left|u\left(y_{p}\right)\right| & \leqslant \sum C \mu_{\Delta}\left(A_{p}\right) \tau^{s(x, y)}\left[\tau^{r_{p}} e^{\varepsilon \omega_{p}}\|u\|_{l}+\frac{1}{\mu_{\Delta}\left(A_{p}\right)} \int_{A_{p}}|u|\right] \\
& \leqslant C \tau^{s(x, y)}\|u\|_{l} \int_{U^{-n} \Delta_{0}} \tau^{\Psi_{n}} e^{\varepsilon \omega}+C \tau^{s(x, y)} \int|u| \\
& \leqslant C \theta^{n} \tau^{s(x, y)}\|u\|_{l}+C \tau^{s(x, y)}\|u\|_{L^{1}}
\end{aligned}
$$

by Lemma 3.6.

To sum up, we have proved that, when $x$ and $y$ belong to the same partition element of the basis,

$$
\frac{\left|\widehat{U}^{n} u(x)-\widehat{U}^{n} u(y)\right|}{\tau^{s(x, y)}} \leqslant C \theta^{n}\|u\|_{l}+C\|u\|_{L^{1}} .
$$


Let now $x$ and $y$ belong to the same element of the partition, with $k=\omega(x)<n$. Let $x^{\prime}$ and $y^{\prime}$ be their projection in the basis. Then

$$
\begin{aligned}
e^{-\varepsilon k} \frac{\left|\widehat{U}^{n} u(x)-\widehat{U}^{n} u(y)\right|}{\tau^{s(x, y)}} & =e^{-\varepsilon k} \frac{\left|\widehat{U}^{n-k} u\left(x^{\prime}\right)-\widehat{U}^{n-k} u\left(y^{\prime}\right)\right|}{\tau^{s\left(x^{\prime}, y^{\prime}\right)}} \leqslant e^{-\varepsilon k}\left[C \theta^{n-k}\|u\|_{l}+C\|u\|_{L^{1}}\right] \\
& \leqslant C \theta^{n}\|u\|_{l}+C\|u\|_{L^{1}} .
\end{aligned}
$$

Assume finally that $k \geqslant n$. Let $x^{\prime}=U^{-n} x$ and $y^{\prime}=U^{-n} y$. Then

$$
e^{-\varepsilon k} \frac{\left|\widehat{U}^{n} u(x)-\widehat{U}^{n} u(y)\right|}{\tau^{s(x, y)}}=e^{-\varepsilon n} e^{-\varepsilon(k-n)} \frac{\left|u\left(x^{\prime}\right)-u\left(y^{\prime}\right)\right|}{\tau^{s\left(x^{\prime}, y^{\prime}\right)}} \leqslant \theta^{n}\|u\|_{l}
$$

Summing up these equations, we get $\left\|\widehat{U}^{n} u\right\|_{l} \leqslant C \theta^{n}\|u\|_{l}+C\|u\|_{L^{1}}$. This concludes the proof of the proposition.

Let $g$ be the locally Hölder function for which we want to prove a limit theorem. It is possible that $g \notin \mathcal{H}$, since $\|g\|_{m}$ is not necessarily finite.

Define a perturbed transfer operator, à la Nagaev, by $\widehat{U}_{t}(u)=\widehat{U}\left(e^{i t g} u\right)$.

Proposition 3.7. There exist constants $C>0$ and $\theta<1$ such that, for all $t \in[-1,1]$, for all $u \in \mathcal{H}$, for all $n \in \mathbb{N}$,

$$
\left\|\widehat{U}_{t}^{n} u\right\| \leqslant C \theta^{n}\|u\|+C\|u\|_{L^{1}} .
$$

This proposition contains Proposition 3.5 as a special case, for $t=0$.

Proof. Let $x \in \Delta$. Then $\widehat{U}_{t}^{n} u(x)=\sum_{U^{n} y=x} e^{i t S_{n} g(y)} J^{(n)}(y) u(y)$, whence $\left|\widehat{U}_{t}^{n} u(x)\right| \leqslant \widehat{U}^{n}|u|(x)$. The bound on $\left\|\widehat{U}^{n}|u|\right\|_{m}$ thus implies the required bound on $\left\|\widehat{U}_{t}^{n} u\right\|_{m}$.

For the Hölder norm, take $x$ and $y$ two points in the same element of partition. Then, with the notations of the proof of Proposition 3.5.

$$
\begin{aligned}
\left|\widehat{U}_{t}^{n} u(x)-\widehat{U}_{t}^{n} u(y)\right| & =\left|\sum e^{i t S_{n} g\left(x_{p}\right)} J^{(n)}\left(x_{p}\right) u\left(x_{p}\right)-e^{i t S_{n} g\left(y_{p}\right)} J^{(n)}\left(y_{p}\right) u\left(y_{p}\right)\right| \\
& \leqslant \sum\left|J^{(n)}\left(x_{p}\right) u\left(x_{p}\right)-J^{(n)}\left(y_{p}\right) u\left(y_{p}\right)\right|+\sum\left|e^{i t S_{n} g\left(x_{p}\right)}-e^{i t S_{n} g\left(y_{p}\right)}\right| J^{(n)}\left(x_{p}\right)\left|u\left(x_{p}\right)\right| .
\end{aligned}
$$

The first sum has already been estimated in the proof of Proposition 3.5. For the second one, $\left|e^{i t S_{n} g\left(x_{p}\right)}-e^{i t S_{n} g\left(y_{p}\right)}\right| \leqslant n C \tau^{s(x, y)}$. Hence, Proposition 3.5] implies that

$$
\left\|\widehat{U}_{t}^{n} u\right\| \leqslant C(n+1) \theta^{n}\|u\|+C(n+1)\|u\|_{L^{1}} .
$$

Choose $N>0$ such that $\bar{\theta}:=C(N+1) \theta^{N}<1$. Iterating the equation $\left\|\widehat{U}_{t}^{N} u\right\| \leqslant \bar{\theta}\|u\|+C\|u\|_{L^{1}}$ (and using the fact that $\left\|\widehat{U}_{t}^{N} u\right\|_{L^{1}} \leqslant\|u\|_{L^{1}}$ ), we get

$$
\left\|\widehat{U}_{t}^{n N} u\right\| \leqslant \bar{\theta}^{n}\|u\|+\frac{C}{1-\bar{\theta}}\|u\|_{L^{1}} .
$$

This implies the conclusion of the proposition, for the constant $\bar{\theta}^{1 / N}<1$.

Lemma 3.8. When $t \rightarrow 0$, $\left\|\widehat{U}_{t}-\widehat{U}\right\|_{\mathcal{H} \rightarrow L^{3}}=O\left(|t|^{1 / 6}\right)$. 
Proof. For $u \in \mathcal{H},\left(\widehat{U}_{t}-\widehat{U}\right) u=\widehat{U}\left(\left(e^{i t g}-1\right) u\right)$. The transfer operator $\widehat{U}$ is a contraction in every $L^{p}$ space, and in particular in $L^{3}$. Hence,

$$
\left\|\left(\widehat{U}_{t}-\widehat{U}\right) u\right\|_{L^{3}} \leqslant\left\|\left(e^{i t g}-1\right) u\right\|_{L^{3}} \leqslant\left\|e^{i t g}-1\right\|_{L^{6}}\|u\|_{L^{6}} .
$$

Note that $\|u\|_{L^{6}} \leqslant C\|u\|$. Hence, $\left\|\widehat{U}_{t}-\widehat{U}\right\|_{\mathcal{H} \rightarrow L^{3}}=O\left(\left\|e^{i t g}-1\right\|_{L^{6}}\right)$. To estimate this quantity, choose $C>0$ such that, for all $x \in \mathbb{R},\left|e^{i x}-1\right| \leqslant C|x|^{1 / 6}$. Then $\int\left|e^{i t g}-1\right|^{6} \leqslant C \int|t g|=O(|t|)$. Hence, $\left\|e^{i t g}-1\right\|_{L^{6}}=O\left(|t|^{1 / 6}\right)$.

\subsubsection{Definition of $\lambda_{t}$, first estimates}

By Proposition 3.5] and Hennion's Theorem [Hen93], the operator $\widehat{U}: \mathcal{H} \rightarrow \mathcal{H}$ is quasicompact: outside of the disk $\{|z| \leqslant \theta\}$, its spectrum is composed of discrete eigenvalues of finite multiplicity. In particular, by ergodicity, 1 is a simple isolated eigenvalue of $\widehat{U}$, with multiplicity one (and the corresponding eigenfunction is the constant function 1).

Lemma 3.8 is not a continuity statement in $\mathcal{H}$. However, the operators $\widehat{U}$ and $\widehat{U}_{t}$ satisfy a uniform Lasota-Yorke inequality between $\mathcal{H}$ and $L^{3}$, by Proposition 3.7 (and the fact that $\|u\|_{L^{1}} \leqslant\|u\|_{L^{3}}$ ). Hence, we can apply the abstract results of [KL99, Corollary 1] (following ideas of [BY93]). We get the following:

For small enough $t, \widehat{U}_{t}$ has a unique eigenvalue $\lambda_{t}$ close to 1 , and it satisfies $\left|\lambda_{t}-1\right|=O\left(|t|^{1 / 10}\right)$. Let $P_{t}$ be the corresponding spectral projection. Then $\left\|P_{t}\right\|_{\mathcal{H} \rightarrow \mathcal{H}}$ is bounded when $t \rightarrow 0$. Moreover, $\left\|P_{t}-P_{0}\right\|_{\mathcal{H} \rightarrow L^{3}}=O\left(|t|^{1 / 10}\right)$.

Remark 3.9. Here, $1 / 10$ could be replaced by any exponent $<1 / 6$, but any positive exponent would be sufficient for our purposes.

Let $\bar{w}_{t}:=P_{t} 1$, and write $w_{t}=\frac{\bar{w}_{t}}{\int \bar{w}_{t}}$. Then $w_{t}$ is bounded in $\mathcal{H}$ and

$$
\left\|w_{t}-1\right\|_{L^{3}}=O\left(|t|^{1 / 10}\right) .
$$

Lemma 3.10. When $t \rightarrow 0$,

$$
\lambda_{t}=1-\frac{t^{2}}{2} \int 1_{|g| \leqslant 1 /|t|} g^{2} w_{t}+i t \int g w_{t}+o\left(t^{2} L(1 /|t|)\right) .
$$

Proof. By definition, $\widehat{U}_{t}\left(w_{t}\right)=\lambda_{t} w_{t}$. Integrating, we get

$$
\lambda_{t}=\int e^{i t g} w_{t} .
$$

We want to use Lemma 3.2 to estimate this integral. However, this lemma applies only to bounded functions. Hence, we will have to modify $w_{t}$.

Take $x \in \Delta$ with $\omega(x)>0$, and let $x^{\prime}=U^{-1}(x)$. The equation $\widehat{U}_{t} w_{t}=\lambda_{t} w_{t}$ implies that $e^{i t g\left(x^{\prime}\right)} w_{t}\left(x^{\prime}\right)=\lambda_{t} w_{t}(x)$. Hence, $\left|w_{t}(x)\right|=\left|\lambda_{t}\right|^{-1}\left|w_{t}\left(x^{\prime}\right)\right|$. Since $w_{t}$ is uniformly bounded on the basis of the tower (since it is bounded in $\mathcal{H}$ ), we get

$$
\left|w_{t}(x)\right| \leqslant C\left|\lambda_{t}\right|^{-\omega(x)} .
$$


Define a function $w_{t}^{\prime}$ by $w_{t}^{\prime}(x)=w_{t}(x)$ if $\omega(x) \leqslant|t|^{-1 / 10}$ and $w_{t}^{\prime}(x)=0$ otherwise. Since $\lambda_{t}=$ $1+O\left(|t|^{1 / 10}\right)$, (25) implies that $w_{t}^{\prime}$ is uniformly bounded.

Lemma 3.2 applied to $w_{t}^{\prime}$ gives

$$
\int\left(e^{i t g}-1-i t g\right) w_{t}^{\prime}=-\frac{t^{2}}{2} \int 1_{|g| \leqslant 1 /|t|} g^{2} w_{t}^{\prime}+o\left(t^{2} L(1 /|t|)\right) .
$$

Let us show that this equation is also satisfied by $w_{t}^{\prime \prime}:=w_{t}-w_{t}^{\prime}$. First,

$$
\left|\int\left(e^{i t g}-1\right) w_{t}^{\prime \prime}\right| \leqslant 2 \int_{\omega \geqslant|t|^{-1 / 10}}\left|w_{t}^{\prime \prime}\right| \leqslant 2 \int_{\omega \geqslant|t|^{-1 / 10}}\left(1+C|t|^{1 / 10}\right)^{\omega} \leqslant C \sum_{n=|t|^{-1 / 10}}^{\infty} \rho^{n}\left(1+C|t|^{1 / 10}\right)^{n} .
$$

When $t$ is small enough, $\rho\left(1+C|t|^{1 / 10}\right)<\sqrt{\rho}<1$. Hence, $\left|\int\left(e^{i t g}-1\right) w_{t}^{\prime \prime}\right| \leqslant C \rho^{|t|^{-1 / 10} / 2}=o\left(t^{2} L(1 /|t|)\right)$. In the same way, $\left|\int g w_{t}^{\prime \prime}\right| \leqslant\|g\|_{L^{3 / 2}}\left\|w_{t}^{\prime \prime}\right\|_{L^{3}}$ and $\left\|w_{t}^{\prime \prime}\right\|_{L^{3}}$ decays stretched exponentially, whence it is $o\left(t^{2} L(1 /|t|)\right)$. Finally,

$$
\left|\int 1_{|g| \leqslant 1 /|t|} g^{2} w_{t}^{\prime \prime}\right| \leqslant \frac{1}{|t|^{2}} \int\left|w_{t}^{\prime \prime}\right|=O\left(\rho^{|t|^{-1 / 10} / 2} / t^{2}\right)=o\left(t^{2} L(1 /|t|)\right) .
$$

Hence, (26) holds also for $w_{t}^{\prime \prime}$. We get

$$
\int\left(e^{i t g}-1-i t g\right) w_{t}=-\frac{t^{2}}{2} \int 1_{|g| \leqslant 1 /|t|} g^{2} w_{t}+o\left(t^{2} L(1 /|t|)\right) .
$$

Since $\int e^{i t g} w_{t}=\lambda_{t}$ and $\int w_{t}=1$, this gives the conclusion of the lemma.

Corollary 3.11. We have $\lambda_{t}=1+O\left(|t|^{11 / 10}\right)$.

Proof. In the proof of the previous lemma, we have proved that $\int_{\omega>|t|-1 / 10} 1_{|g| \leqslant 1 /|t|} g^{2} w_{t}=O(t)$. Moreover, on $\left\{\omega \leqslant|t|^{-1 / 10}\right\}$, the function $w_{t}$ is uniformly bounded. Hence, $\int_{\omega \leqslant|t|-1 / 10} 1_{|g| \leqslant 1 /|t|} g^{2} w_{t} \leqslant$ $C \int 1_{|g| \leqslant 1 /|t|} g^{2} \sim C L(1 /|t|)$. Hence,

$$
\int 1_{|g| \leqslant 1 /|t|} g^{2} w_{t}=O(L(1 /|t|))
$$

Moreover, by (22) and our assumption $\int g=0$,

$$
\left|\int g w_{t}\right|=\left|\int g\left(w_{t}-1\right)\right| \leqslant\|g\|_{L^{3 / 2}}\left\|w_{t}-1\right\|_{L^{3}}=O\left(|t|^{1 / 10}\right) .
$$

This proves that, in (23), the right side is $1+O\left(|t|^{11 / 10}\right)$.

\subsubsection{Estimates on the basis}

To proceed, we will need to know that $w_{t}$ is constant on the basis up to $O(t)$. We already know that $\left\|w_{t}-1\right\|_{L^{3}}=O\left(|t|^{1 / 10}\right)$, but this is not sufficient to estimate precisely the terms in (23). To get such an estimate, we will need real continuity, and not only the weak continuity given by Keller-Liverani's theorem. This will be achieved by working directly on the basis. The goal of this paragraph is to prove Lemma 3.15. 
Let $U_{0}$ be the map induced by $U$ on the basis $\Delta_{0}$ of the tower. Denote by $\varphi$ the first return time to the basis, so that $U_{0}(x)=U^{\varphi(x)}(x)$.

Let us consider the space $\mathcal{H}_{0}$ of Hölder functions $u: \Delta_{0} \rightarrow \mathbb{C}$ on the basis, and define an operator $R_{n}: \mathcal{H}_{0} \rightarrow \mathcal{H}_{0}$ by $R_{n} u(x)=\sum J^{(n)}(y) u(y)$, where the sum is restricted to those $y \in \Delta_{0}$ with return time $\varphi(y)=n$, and $U^{n}(y)=x$. Set also $R_{n}(t)(u)=R_{n}\left(e^{i t S_{n} g} u\right)$.

Lemma 3.12. There exist $C>0$ and $\theta<1$ such that, for all $n \in \mathbb{N}$ and all $t \in[-1,1],\left\|R_{n}(t)\right\| \leqslant$ $C \theta^{n}$ and $\left\|R_{n}(t)-R_{n}\right\| \leqslant C \theta^{n}|t|$.

Proof. The map $U_{0}$ is Gibbs-Markov on $\Delta_{0}$. Hence, Gou04, Lemma 3.2] proves that $\left\|R_{n}\right\| \leqslant$ $C \mu_{\Delta}(\varphi=n)$ and [Gou04, Lemma 3.5] yields $\left\|R_{n}(t)-R_{n}\right\| \leqslant C|t| n \mu_{\Delta}(\varphi=n)+C \int_{\varphi=n}\left|e^{i t S_{n} g}-1\right|$.

Since $\mu_{\Delta}(\varphi=n)=O\left(\rho^{n}\right)$, we get in particular $\left\|R_{n}\right\| \leqslant C \rho^{n}$, which decays exponentially. Moreover, on $\{\varphi=n\},\left|S_{n} g\right|^{3 / 2} \leqslant n^{1 / 2} \sum_{k=0}^{n-1}\left|g \circ U^{k}\right|^{3 / 2}$, whence $\int_{\varphi=n}\left|S_{n} g\right|^{3 / 2} \leqslant n^{1 / 2} \int_{\Delta}|g|^{3 / 2}=O\left(n^{1 / 2}\right)$. Consequently,

$$
\left\|R_{n}(t)-R_{n}\right\| \leqslant C|t| n \rho^{n}+C \int 1_{\varphi=n}|t|\left|S_{n} g\right| \leqslant C|t| n \rho^{n}+C|t|\left\|1_{\varphi=n} S_{n} g\right\|_{L^{3 / 2}}\left\|1_{\varphi=n}\right\|_{L^{3}},
$$

which decays also exponentially.

For $|z|<\theta^{-1}$, it is possible to define $R(z, t):=\sum z^{n} R_{n}(t)$. The operator $R(1,0)$ is the transfer operator associated to $U_{0}$. It has a simple isolated eigenvalue at 1 , and the corresponding eigenfunction is the constant function 1 . Hence, for $(z, t)$ close enough to $(1,0), R(z, t)$ has a unique eigenvalue $\lambda(z, t)$ close to 1 .

Lemma 3.13. We have $\|R(z, t)-R(1,0)\|=O(|t|+|z-1|)$.

Proof. We have $R(z, t)-R(1, t)=\sum\left(z^{n}-1\right) R_{n}(t)$. Moreover, if $|z| \leqslant \theta^{-1 / 2},\left|z^{n}-1\right| \leqslant \mid z-$ $\left.1\left|\sum_{k=0}^{n-1}\right| z\right|^{k} \leqslant C|z-1| \theta^{-n / 2}$. Hence,

$$
\|R(z, t)-R(1, t)\| \leqslant \sum_{n=0}^{\infty}\left|z^{n}-1\right|\left\|R_{n}(t)\right\| \leqslant C|z-1| \sum_{n=0}^{\infty} \theta^{-n / 2} \theta^{n} \leqslant \frac{C}{1-\theta^{1 / 2}}|z-1| .
$$

Moreover,

$$
\|R(1, t)-R(1,0)\| \leqslant \sum_{n=0}^{\infty}\left\|R_{n}(t)-R_{n}\right\| \leqslant \sum_{n=0}^{\infty} C|t| \theta^{n} \leqslant \frac{C}{1-\theta}|t| .
$$

Lemma 3.14. We have $R\left(\lambda_{t}^{-1}, t\right)\left(1_{\Delta_{0}} w_{t}\right)=1_{\Delta_{0}} w_{t}$.

Proof. Let $x \in \Delta_{0}$, let $\left\{x_{p}\right\}$ be the set of its preimages under $U$, at respective heights $\omega_{p}$, and let $x_{p}^{\prime}$ be the projection of $x_{p}$ in the basis. Since $\widehat{U}_{t} w_{t}=\lambda_{t} w_{t}$, we have $\lambda_{t} w_{t}(x)=\sum e^{i t g\left(x_{p}\right)} J\left(x_{p}\right) w_{t}\left(x_{p}\right)$. Moreover, for any $y \in \Delta$ with $\omega(y)>0$, we have $\lambda_{t} w_{t}(y)=e^{i t g\left(U^{-1} y\right)} w_{t}\left(U^{-1} y\right)$. Hence, $\lambda_{t}^{\omega_{p}} w_{t}\left(x_{p}\right)=$ $e^{i t S_{\omega_{p}} g\left(x_{p}^{\prime}\right)} w_{t}\left(x_{p}^{\prime}\right)$. We get

$$
w_{t}(x)=\sum \lambda_{t}^{-\omega_{p}-1} J^{\left(\omega_{p}+1\right)}\left(x_{p}^{\prime}\right) e^{i t S_{\omega_{p}+1} g\left(x_{p}^{\prime}\right)} w_{t}\left(x_{p}^{\prime}\right) .
$$

The points $x_{p}^{\prime}$ are exactly the preimages of $x$ under $U_{0}$, and the corresponding return time for $U$ is $\omega_{p}+1$. Hence, (27) gives the conclusion of the lemma. 
We have all the necessary tools to prove the main result of this paragraph:

Lemma 3.15. For $t \in[-1,1]$, there exists $c(t) \in \mathbb{C}$ such that $\left\|1_{\Delta_{0}}\left(w_{t}-c(t)\right)\right\|_{\infty}=O(t)$. Moreover, $c(t) \rightarrow 1$ when $t \rightarrow 0$.

Proof. Lemma 3.14 proves that $\lambda\left(\lambda_{t}^{-1}, t\right)=1$, and the corresponding eigenfunction is $1_{\Delta_{0}} w_{t}$. Let $Q_{t}$ be the eigenprojection of $R\left(\lambda_{t}^{-1}, t\right)$ corresponding to the eigenvalue 1 . It satisfies

$$
\left\|Q_{t}-Q_{0}\right\|=O\left(\left\|R\left(\lambda_{t}^{-1}, t\right)-R(1,0)\right\|\right)=O\left(\left|\lambda_{t}^{-1}-1\right|+|t|\right)=O(|t|)
$$

by Lemma 3.13 and Corollary 3.11. Let $b_{t}=Q_{t} 1_{\Delta_{0}}$. As $b_{0}=1_{\Delta_{0}}, b_{t}$ satisfies $\left\|b_{t}-1_{\Delta_{0}}\right\|=O(t)$. In particular, $b_{t} \rightarrow 1_{\Delta_{0}}$ in $L^{1}$.

The function $b_{t}$ is proportional to $w_{t}$ on the basis $\Delta_{0}$. Hence, there exists a scalar $c(t)$ such that $1_{\Delta_{0}} w_{t}=c(t) b_{t}$. Since $w_{t}$ goes to 1 in $L^{1}$ when $t \rightarrow 0$, we get

$$
c(t)=\frac{\int 1_{\Delta_{0}} w_{t}}{\int b_{t}} \rightarrow \frac{\mu_{\Delta}\left(\Delta_{0}\right)}{\int b_{0}}=1
$$

Finally,

$$
\left\|1_{\Delta_{0}}\left(w_{t}-c(t)\right)\right\|_{\infty}=|c(t)|\left\|b_{t}-b_{0}\right\|_{\infty}=O(t) .
$$

\subsubsection{Proof of Theorem $\left[3.4\right.$ when $\int g=0$}

Let the function $G$ be given by $G(x)=\sum_{k=0}^{\omega(x)-1} g\left(U^{k} \pi_{0} x\right)$, as in Theorem 3.4

Lemma 3.16. When $t \rightarrow 0$,

$$
\lambda_{t}=1-(1+o(1)) \frac{t^{2}}{2} \int 1_{|g| \leqslant 1 /|t|} g^{2} e^{i t G}+i t(1+o(1)) \int g e^{i t G}+o\left(t^{2} L(1 /|t|)\right) .
$$

Proof. We will start from (23) and show that we can replace $w_{t}$ by $e^{i t G}$.

We have $w_{t}(x)=\lambda_{t}^{-\omega(x)} e^{i t G(x)} w_{t}\left(\pi_{0} x\right)$. Hence, by Corollary 3.11 and Lemma 3.15

$$
\begin{aligned}
\left|w_{t}(x)-c(t) e^{i t G(x)}\right| & =\left|\lambda_{t}^{-\omega(x)} w_{t}\left(\pi_{0} x\right)-c(t)\right| \leqslant\left|\lambda_{t}^{-\omega(x)}-1\right|\left|w_{t}\left(\pi_{0} x\right)\right|+\left|w_{t}\left(\pi_{0} x\right)-c(t)\right| \\
& \leqslant\left[\left(1+C|t|^{11 / 10}\right)^{\omega(x)}-1\right] C+C|t| \leqslant \omega(x) C|t|^{11 / 10}\left(1+C|t|^{11 / 10}\right)^{\omega(x)}+C|t| .
\end{aligned}
$$

Fix $b>0$ large enough. For $\omega(x) \leqslant b \log (1 /|t|)$, we obtain $\left|w_{t}(x)-c(t) e^{i t G(x)}\right| \leqslant C|t|$. For $\omega(x) \geqslant$ $b \log (1 /|t|)$ and small enough $t$, we also get $\left|w_{t}(x)-c(t) e^{i t G(x)}\right| \leqslant \rho^{-\omega(x) / 4}$.

Hence,

$$
\int_{\omega \geqslant b \log (1 /|t|)} 1_{|g| \leqslant 1 /|t|} g^{2}\left|w_{t}-c(t) e^{i t G}\right| \leqslant \int_{\omega \geqslant b \log (1 /|t|)} \frac{1}{|t|^{2}} \rho^{-\omega(x) / 4} \leqslant \frac{1}{|t|^{2}} C \sum_{n=b \log (1 /|t|)}^{\infty} \rho^{n} \rho^{-n / 4}=o(1)
$$

if $b$ is large enough. Moreover,

$$
\int_{\omega \leqslant b \log (1 /|t|)} 1_{|g| \leqslant 1 /|t|} g^{2}\left|w_{t}-c(t) e^{i t G}\right| \leqslant C|t| \int 1_{|g| \leqslant 1 /|t|} g^{2}=C|t| L(1 /|t|) .
$$


Hence,

$$
\int 1_{|g| \leqslant 1 /|t|} g^{2} w_{t}=c(t) \int 1_{|g| \leqslant 1 /|t|} g^{2} e^{i t G}+o(1)=(1+o(1)) \int 1_{|g| \leqslant 1 /|t|} g^{2} e^{i t G}+o(1) .
$$

In the same way,

$$
\int_{\omega \geqslant b \log (1 /|t|)}|g|\left|w_{t}-c(t) e^{i t G}\right| \leqslant\|g\|_{3 / 2}\left\|1_{\omega \geqslant b \log (1 /|t|)}\left|w_{t}-c(t) e^{i t G}\right|\right\|_{L^{3}}=O(t)
$$

if $b$ is large enough. Moreover,

$$
\int_{\omega \leqslant b \log (1 /|t|)}|g|\left|w_{t}-c(t) e^{i t G}\right| \leqslant \int|g| C|t|=O(t)
$$

We get

$$
\int g w_{t}=c(t) \int g e^{i t G}+O(t)=(1+o(1)) \int g e^{i t G}+O(t) .
$$

Equations (29) and (30) together with (23) imply (28).

Remark 3.17. The proof of the lemma also shows that, in (28), it is sufficient to integrate on $\{\omega(x) \leqslant b \log (1 /|t|)\}$ if $b$ is large enough, since the remaining part is in $o\left(t^{2} L(1 /|t|)\right)$.

The following lemma will use the additional assumptions that $l$ is slowly varying and $l(x \ln x) \sim l(x)$, $L(x \ln x) \sim L(x)$.

Lemma 3.18. We have

$$
\int 1_{|g| \leqslant 1 /|t|} g^{2} e^{i t G}=L(1 /|t|)(1+o(1))
$$

Proof. It is sufficient to prove (31) on $\{\omega \leqslant b \log (1 /|t|)\}$, since the remaining part can be ignored. Take some $\varepsilon>0$, we will prove that

$$
\int_{\omega \leqslant b \log (1 /|t|)} 1_{|g| \leqslant 1 /|t|} g^{2}\left|e^{i t G}-1\right| \leqslant 2 \varepsilon L(1 /|t|)
$$

when $t$ is small enough. This will be sufficient to conclude the proof.

Let $A_{t}:=\{x|\omega(x) \leqslant b \log (1 /|t|)| G,(x)|\geqslant \varepsilon /| t \mid\}$. if $x \in A_{t}$, there exists $y$ below $x$ in the tower such that $|g(y)| \geqslant \frac{\varepsilon}{|t| b \log (1 /|t|)}$. Let $B=\left\{x|| g(x) \mid \geqslant \frac{\varepsilon}{|t| b \log (1 /|t|)}\right\}$, we get $\mu_{\Delta}\left(A_{t}\right) \leqslant b \log (1 /|t|) \mu_{\Delta}(B)$.

Let $Z$ be a random variable on $\mathbb{R}$ with the distribution of $g$. Then

$$
\begin{aligned}
P\left(\frac{1}{|t| \log (1 /|t|)^{2}} \leqslant|Z| \leqslant 1 /|t|\right) & =|t|^{2} \log (1 /|t|)^{4} l\left(\frac{1}{|t| \log (1 /|t|)^{2}}\right)-|t|^{2} l(1 /|t|) \\
& =|t|^{2} \log (1 /|t|)^{4} l(1 /|t|)(1+o(1))
\end{aligned}
$$

and

$$
\begin{aligned}
b \log (1 /|t|) P\left(|Z| \geqslant \frac{\varepsilon}{|t| b \log (1 /|t|)}\right) & =b \log (1 /|t|) \frac{|t|^{2} b^{2} \log (1 /|t|)^{2}}{\varepsilon^{2}} l\left(\frac{\varepsilon}{|t| b \log (1 /|t|)}\right) \\
& =\frac{|t|^{2} b^{3} \log (1 /|t|)^{3}}{\varepsilon^{2}} l(1 /|t|)(1+o(1)) .
\end{aligned}
$$


Hence, if $t$ is small enough, $\mu_{\Delta}\left(A_{t}\right) \leqslant P\left(\frac{1}{|t| \log (1 /|t|)^{2}} \leqslant|Z| \leqslant 1 /|t|\right)$.

We would like to estimate $\int_{A_{t}} 1_{|g| \leqslant 1 /|t|} g^{2}$. Now

$$
\int_{A_{t}} 1_{|g| \leqslant 1 /|t|} g^{2}=\int_{A_{t}} 1_{\frac{1}{|t| \log (1 /|t|)^{2}}<|g| \leqslant 1 /|t|} g^{2}+\int_{A_{t}} 1_{|g| \leqslant \frac{1}{|t| \log (1 /|t|)^{2}}} g^{2} .
$$

On the one hand

$$
\int_{A_{t}} 1 \frac{1}{|t| \log (1 /|t|)^{2}}<|g| \leqslant 1 /|t| g^{2} \leqslant \int_{\frac{1}{|t| \log (1 /|t|)^{2}}}^{1 /|t|} x^{2} \mathrm{~d} P(x),
$$

and on the other hand, by applying the above bounds we get

$$
\begin{aligned}
\int_{A_{t}} 1_{|g| \leqslant \frac{1}{|t| \log (1 /|t|)^{2}}} g^{2} & \leqslant \frac{1}{|t|^{2} \log (1 /|t|)^{4}} \mu_{\Delta}\left(A_{t}\right) \\
& \leqslant \frac{1}{|t|^{2} \log (1 /|t|)^{4}} P\left(\frac{1}{|t| \log (1 /|t|)^{2}} \leqslant|Z| \leqslant 1 /|t|\right) \\
& \leqslant \int_{\frac{1}{|t| \log (1 /|t|)^{2}}}^{1 /|t|} x^{2} \mathrm{~d} P(x) .
\end{aligned}
$$

Thus we need to deal with the integral

$$
\int_{\frac{1}{|t| \log (1 /|t|)^{2}}}^{1 /|t|} x^{2} \mathrm{~d} P(x)
$$

which is equal to

$$
L(1 /|t|)-L\left(\frac{1}{|t| \log (1 /|t|)^{2}}\right)=L(1 /|t|)-L(1 /|t|)(1+o(1))=o(L(1 /|t|)) .
$$

Hence, for small enough $t$, we get

$$
\int_{A_{t}} 1_{|g| \leqslant 1 /|t|} g^{2}\left|e^{i t G}-1\right| \leqslant \varepsilon L(1 /|t|) .
$$

On $B_{t}:=\left\{x \mid \omega(x) \leqslant b \log (1 /|t|), x \notin A_{t}\right\}$, we have $\left|e^{i t G(x)}-1\right| \leqslant|t||G(x)| \leqslant \varepsilon$. Hence,

$$
\int_{B_{t}} 1_{|g| \leqslant 1 /|t|} g^{2}\left|e^{i t G}-1\right| \leqslant \varepsilon \int 1_{|g| \leqslant 1 /|t|} g^{2}=\varepsilon L(1 /|t|) .
$$

These two equations imply (32). This concludes the proof.

Since $\int g=0$, Lemma 3.16 gives

$$
\begin{aligned}
\lambda_{t} & =1-\frac{t^{2}}{2} L(1 /|t|)(1+o(1))+(1+o(1)) i t \int g e^{i t G} \\
& =1-\frac{t^{2}}{2} L(1 /|t|)(1+o(1))+(1+o(1)) i t \int g\left(e^{i t G}-1\right) \\
& =1-\frac{t^{2}}{2} L_{1}(1 /|t|)(1+o(1)),
\end{aligned}
$$

since $\int g\left(e^{i t G}-1\right)=i(a+o(1)) t L(1 /|t|)$ by assumption.

This asymptotic expansion readily implies the conclusion of Theorem 3.4 , for $g$ such that $\int g=0$. 


\subsubsection{Proof of Theorem 3.4 in the general case}

Let $g: \Delta \rightarrow \mathbb{R}$ belong to $L^{p}(\Delta)$ for any $p<2$ (this is in particular the case if $g$ satisfies the assumptions of Theorem 3.4). Set $G(x)=\sum_{k=0}^{\omega(x)-1} g\left(U^{k} \pi_{0} x\right)$.

Lemma 3.19. For any $p<2$, the function $G$ belongs to $L^{p}(\Delta)$.

Proof. For $x \in \Delta$, let $\varphi(x)$ be its return time to the basis. Set also $\psi(x)=\varphi\left(\pi_{0} x\right)$, where $\pi_{0}$ is the projection on the basis of the tower.

We have

$$
|G(x)|^{p}=\left|\sum_{k=0}^{\omega(x)-1} g\left(U^{k} \pi_{0} x\right)\right|^{p} \leqslant \omega(x)^{p-1} \sum_{k=0}^{\omega(x)-1}\left|g\left(U^{k} \pi_{0} x\right)\right|^{p} .
$$

Changing variables, we get

$$
\int|G(x)|^{p} \leqslant \int|g(y)|^{p} \sum_{k=1}^{\varphi(y)-1} \omega\left(U^{k} y\right)^{p-1} \leqslant \int|g(y)|^{p} \psi(y)^{p} .
$$

Since the tower has exponentially small tails, the function $\psi$ belongs to $L^{q}$ for any $q<\infty$. Using the Hölder inequality with a sufficiently large $q$, we obtain $\int|G(x)|^{p}<\infty$.

Let $g^{\prime}$ be another function on $\Delta$. Define also $G^{\prime}(x)=\sum_{k=0}^{\omega(x)-1} g^{\prime}\left(U^{k} \pi_{0} x\right)$.

Lemma 3.20. if $g-g^{\prime}$ is bounded, then

$$
\int g\left(e^{i t G}-1\right)=\int g^{\prime}\left(e^{i t G^{\prime}}-1\right)+O(t)
$$

when $t \rightarrow 0$.

Proof. We have

$$
\int g\left(e^{i t G}-1\right)-\int g^{\prime}\left(e^{i t G^{\prime}}-1\right)=\int\left(g-g^{\prime}\right)\left(e^{i t G^{\prime}}-1\right)+\int g\left(e^{i t G}-e^{i t G^{\prime}}\right) .
$$

Since $g-g^{\prime}$ is bounded, the first integral satisfies

$$
\left|\int\left(g-g^{\prime}\right)\left(e^{i t G^{\prime}}-1\right)\right| \leqslant C|t| \int\left|G^{\prime}\right|,
$$

which is $O(t)$ since $G^{\prime}$ is integrable by Lemma 3.19, For the second integral, $\left|G(x)-G^{\prime}(x)\right| \leqslant C \omega(x)$. Hence,

$$
\left|\int g\left(e^{i t G}-e^{i t G^{\prime}}\right)\right| \leqslant \int|g| C|t| \omega \leqslant C|t|\|g\|_{L^{3 / 2}}\|\omega\|_{L^{3}}=O(t) .
$$

Proof of Theorem 3.4. Let $g$ satisfy the assumptions of Theorem 3.4. Write $g^{\prime}=g-\int g$. Then $g^{\prime}$ is still in the nonstandard domain of attraction of the normal law, and its distribution functions $l^{\prime}$ and $L^{\prime}$ satisfy $l^{\prime} \sim l$ and $L^{\prime} \sim L$. Moreover,

$$
\left.\int g^{\prime}\left(e^{i t G^{\prime}}-1\right)=(a+o(1)) i t L(1 /|t|)\right),
$$


since $g$ satisfies the same estimate and Lemma 3.20 applies.

We have already proved Theorem 3.4 for functions of zero integral. This applies to $g^{\prime}$, and gives $\frac{S_{n} g^{\prime}}{B_{n}} \rightarrow \mathcal{N}(0,1)$. Since $S_{n} g^{\prime}=S_{n} g-n \int g$, this concludes the proof.

Remark 3.21. Lemmas 3.19 and 3.20 do not involve the dynamics of the returns to the basis. Hence, they also hold in hyperbolic Young towers.

\section{Estimate of the integral in the stadium billiard}

Let us turn back to the study of the stadium. We will use the notations of the first two sections. In particular, starting from a fixed function $f_{0}: X_{0} \rightarrow \mathbb{R}$ satisfying $(P 1)$, we have obtained a function $g: \Delta \rightarrow \mathbb{R}$. According to Theorem 3.4. if we want to obtain a limit theorem for $g$, the quantity to be estimated is $\int g\left(e^{i t G}-1\right)$. The main result of this section is the following proposition.

Proposition 4.1. Let $y=\frac{1}{1-\frac{3}{4} \log 3}$, and recall the definition of I from (11). We have

$$
\int_{\Delta} g\left(e^{i t G}-1\right) \mathrm{d} \mu_{\Delta}=i \frac{I^{2}(y-1) \ell^{2}}{\pi} t \log (1 /|t|)+o(t \log (1 /|t|))
$$

Our proof approximates the left hand side with an integral explicitly given in the phase space of the stadium. This later integral can be estimated with sufficient precision due to the geometric properties of the billiard map.

\subsection{Preliminary estimates}

First we show that the relevant expression can be pulled back to the hyperbolic Young tower. Let $\omega(x)$ be the height of the point $x$ in $\bar{\Delta}$, and let $\bar{\pi}_{0}: \bar{\Delta} \rightarrow \bar{\Delta}_{0}$ be the projection on the basis. We define two functions $\bar{F}$ and $\bar{G}$ on $\bar{\Delta}$ by $\bar{F}(x)=\sum_{k=0}^{\omega(x)-1} \bar{f}\left(\bar{U}^{k} \bar{\pi}_{0} x\right)$ and $\bar{G}(x)=\sum_{k=0}^{\omega(x)-1} \bar{g}\left(\bar{U}^{k} \bar{\pi}_{0} x\right)$.

Lemma 4.2. We have

$$
\int_{\Delta} g\left(e^{i t G}-1\right)=\int_{\bar{\Delta}} \bar{f}\left(e^{i t \bar{F}}-1\right)+O(t)
$$

Proof. As $\left(\pi_{\Delta}\right)_{*}\left(\mu_{\bar{\Delta}}\right)=\mu_{\Delta}, \int_{\Delta} g\left(e^{i t G}-1\right)=\int_{\bar{\Delta}} \bar{g}\left(e^{i t \bar{G}}-1\right)$ automatically. As $\bar{g}-\bar{f}$ is bounded by Lemma 2.4, Lemma 3.20 implies the statement.

Note that $\bar{F}$ is essentially a Birkhoff sum of $f$ for the inverse map $T^{-1}$. Thus, if we switch from $T$ to $T^{-1}$, we may investigate our integral by dynamical tools.

For all $x \in X$, let $h(x)=f\left(T^{-1} x\right)$. Introduce $\bar{h}=h \circ \pi_{X}$. For $x \in \bar{\Delta}$ with $\omega(x)>0$, let $\bar{H}(x)=\sum_{k=1}^{\omega(x)-1} \bar{h}\left(\bar{U}^{-k} x\right)$, or equivalently, $\bar{H}(x)=\bar{F}\left(\bar{U}^{-1} x\right)$. We fix $\bar{H}(x)=0$ on $\Delta_{0}$.

Lemma 4.3. We have

$$
\int \bar{f}\left(e^{i t \bar{F}}-1\right)=\int \bar{h}\left(e^{i t \bar{H}}-1\right)+O(t)
$$


Proof. We have $\bar{h} \circ \bar{U}=\bar{f}$, and, apart from $\bar{U}^{-1}\left(\bar{\Delta}_{0}\right), \bar{H} \circ \bar{U}=\bar{F}$. Thus,

$$
\begin{aligned}
\int \bar{h}\left(e^{i t \bar{H}}-1\right)-\int \bar{f}\left(e^{i t \bar{F}}-1\right) & =\int \bar{h} \circ \bar{U}\left(e^{i t \bar{H} \circ \bar{U}}-1\right)-\int \bar{f}\left(e^{i t \bar{F}}-1\right) \\
& =\int_{\bar{U}^{-1}\left(\bar{\Delta}_{0}\right)}\left[\bar{h} \circ \bar{U}\left(e^{i t \bar{H} \circ \bar{U}}-1\right)-\bar{f}\left(e^{i t \bar{F}}-1\right)\right] .
\end{aligned}
$$

As $\varphi_{+}$is bounded on the rectangle $R$ that defines the basis of the tower, the functions $\bar{h} \circ \bar{U}$ and $\bar{f}$ are bounded on $\bar{U}^{-1}\left(\bar{\Delta}_{0}\right)$. By Lemma $3.19 \bar{F}$ and $\bar{H}$ are both integrable. This completes the proof.

We will consider $T^{-1}$ as the first return map of $T_{0}^{-1}$ to the subspace $X$. The return time is $\varphi_{-}=$ $\varphi_{+} \circ T^{-1}$.

\subsection{Geometric properties of $T^{-1}$ in the vicinity of its singularities}

The behavior of $\int \bar{h}\left(e^{i t \bar{H}}-1\right)$ is governed by the dynamical properties of $T^{-1}$ at those parts of the phase space where it is equivalent to a long series of bounces along the parallel segments of the boundary. These sets have the following structure: the points for which $T^{-1}$ acts as $n$ consecutive bounces on the segments form two stripes of slope approximately -1 . $T^{-1}$ maps these two cells onto two stripes of positive slope. The geometry is described on the figure below.

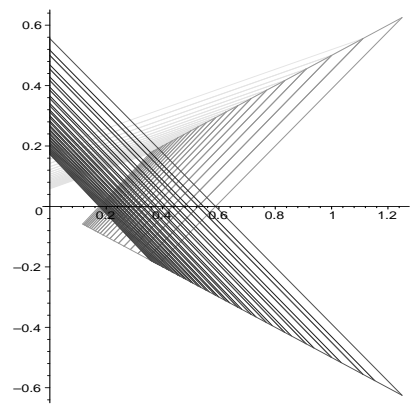

More precisely, this figure appears four times in $X$ (twice in both of the parallelograms that define $X)$. The transformation $T^{-1}$ jumps from one such region to another, however, they play the same dynamical role. Thus, to simplify matters, we pretend as if we had only one of them.

Recall that $\varphi_{-}=\varphi_{+} \circ T^{-1}$ is the return time of $T_{0}^{-1}$ to $X$. As a further notational simplification we ignore that there are two stripes on which $\left\{\varphi_{-}=n\right\}$. Let $M_{n}$ stand for the stripe $\left\{\varphi_{-}=n\right\}$, which will be also referred to as the set of points of return time $n$.

Later on we shall see that the other type of singularities (corresponding to trajectories sliding along the semi-circle) does not play any role in the leading term behavior of $\int \bar{h}\left(e^{i t \bar{H}}-1\right)$.

Remark 4.4. We need to study the map $T^{-1}$ and not $T$. These two are not isomorphic, as $X$ is the set of points on a semi-circle for which the previous collision is not on that semi-circle. This definition introduces an asymmetry of past and future. The map $T^{-1}$ is, however, isomorphic to the map induced on the set of points on a semi-circle for which the next collision is on another semi-circle. This later induced map has been studied by Markarian in Mar04, where he, in particular, has shown that it satisfies Chernov's axioms from [Che99]. 
Fix $\rho<1$ such that the tails of the original Young tower are bounded by $c \rho^{n}$, and $K>0$ such that $K \log \left(\rho^{-1}\right)>4$. In what follows we essentially consider curves with tangent vectors in the unstable cone of $T^{-1}$ (u-curves of $T^{-1}$ for short).

Definition 4.5. Consider the stripe $M_{n}$ and its two sides of slope -1 . A good curve $\mathcal{C}$ of return time $n$ is a $C^{1}$ curve that connects these two sides. We put further requirements on the slope of $\mathcal{C}$ : it should belong to the interval $[1 / 4,4]$ for all points and it should be constant up to $1 / \sqrt{n}$ precision (i.e., for all points $x$ and $y$ in $\mathcal{C}$, the slopes of $\mathcal{C}$ at $x$ and $y, s(x)$ and $s(y)$, should satisfy $\left.|s(x)-s(y)| \leqslant \frac{1}{\sqrt{n}}\right)$.

Remark 4.6. Note that our requirement on the slope in not a curvature bound. Stated in this form, it is not hard to see that good curves tend to have more and more constant slopes when iterated by $T^{-1}$. To see this consider a good curve with large return time $n$ and iterate it backwards by the billiard flow. Just before collision it corresponds to a dispersing wavefront that defocuses within finite time and, while experiencing many bounces with the straight walls, loses most of its curvature. At the time moment just after the previous collision on the other semi-circle, this wavefront is flat up to $1 / n$. Thus, any subcurve $\mathcal{C}^{\prime} \subset T^{-1} \mathcal{C}$ is automatically a good curve if it connects the two long sides of a stripe.

Definition 4.7. A standard curve is defined as a good curve of constant slope 1. In particular, it is a segment.

The choice of 1 as the slope for standard curves is arbitrary. More important is the fact that the standard curves of return time $n$ give a fixed foliation for (most of) the stripe $M_{n}$.

If $\mathcal{C}$ is a good curve of return time $n$, any point of $T^{-1}(\mathcal{C})$ has return time at least $n / 3-C_{1}$ and at most $3 n+C_{1}$ for some constant $C_{1}$. Furthermore, there exists a constant $C_{2} \in \mathbb{N}$ such that for any $i \in\left[n / 3+C_{2}, 3 n-C_{2}\right]$, the set $T^{-1}(\mathcal{C}) \cap\left\{\varphi_{-}=i\right\}$ is a good curve of return time $i$ (see Remark 4.6). Let us denote $\mathcal{C}_{i}=\left\{x \in \mathcal{C} \mid \varphi_{-}\left(T^{-1} x\right)=i\right\}$. We also have

$$
\frac{\operatorname{Leb}\left(\mathcal{C} \backslash \bigcup_{i \in\left[n / 3+C_{2}, 3 n-C_{2}\right]} \mathcal{C}_{i}\right)}{\operatorname{Leb}(\mathcal{C})} \leqslant \frac{C}{n}
$$

for a universal constant $C$. We will say that the set $\mathcal{C} \backslash \bigcup_{i \in\left[n / 3+C_{2}, 3 n-C_{2}\right]} \mathcal{C}_{i}$ is thrown away at the first iterate of $\mathcal{C}$. Formula (33) shows that the points which are thrown away have negligible measure.

Remark 4.8. In addition to the above observations, it is possible to estimate the transition probabilities from one stripe to the other in the following sense. There exists a sequence $\varepsilon_{n}$ that tends to 0 as $n \rightarrow \infty$, such that for any good curve $\mathcal{C}$ of return time $n$, and for any $i \in\left[n / 3+C_{2}, 3 n-C_{2}\right]$,

$$
\left(1-\varepsilon_{n}\right) \frac{3 n}{8 i^{2}} \leqslant \frac{\operatorname{Leb}\left\{x \in \mathcal{C} \mid \varphi_{-}\left(T^{-1}(x)\right)=i\right\}}{\operatorname{Leb}(\mathcal{C})} \leqslant \frac{3 n}{8 i^{2}}\left(1+\varepsilon_{n}\right) .
$$

This can be verified by direct calculation. In other words, we go from $n$ to $i$ asymptotically with probability $\frac{3 n}{8 i^{2}}$ (note that $\sum_{i=n / 3+C_{2}}^{3 n-C_{2}} \frac{3 n}{8 i^{2}} \rightarrow 1$ ).

Applying the above process several times, we may iterate the good curves by $T^{-1}$ and obtain finer and finer partitions of $\mathcal{C}$. A sequence of integers $n_{0}, n_{1}, \ldots, n_{k}$ is referred to as admissible if, for all $i<k, n_{i+1} \in\left[n_{i} / 3+C_{2}, 3 n_{i}-C_{2}\right]$. Given a good curve of return time $n_{0}, \mathcal{C}$, and an admissible sequence $n_{0}, \ldots, n_{k}$, let

$$
\mathcal{C}_{n_{0}, \ldots, n_{k}}=\left\{x \in \mathcal{C} \mid \forall i \leqslant k, \varphi_{-}\left(T^{-i} x\right)=n_{i}\right\}
$$

This is a subcurve of $\mathcal{C}$ mapped by $T^{-k}$ onto a good curve of return time $n_{k}$. 
Lemma 4.9. There exists a constant $C>0$ such that, for any pair of good curves of the same return time $n_{0}, \mathcal{C}$ and $\mathcal{C}^{\prime}$, and for any fixed admissible sequence $n_{0}, \ldots, n_{k}$, we have

$$
C^{-1} \leqslant \frac{\operatorname{Leb}\left(\mathcal{C}_{n_{0}, \ldots, n_{k}}\right)}{\operatorname{Leb}\left(\mathcal{C}_{n_{0}, \ldots, n_{k}}^{\prime}\right)} \leqslant C .
$$

Proof. This follows from the uniform expansion and the bounded distortion properties of $T^{-1}$ along its u-curves.

In what follows, when we talk about iterating a good curve, we will always mean the above process of refinement, along with throwing away some part at each step. However, the number of iterations may depend on the point of $\mathcal{C}$ we are considering. This is formulated in the following definition.

Definition 4.10. Let $\mathcal{C}$ be a good curve of return time $n$. Let furthermore $A$ be a subset of $\mathcal{C}$ and $\tau: \mathcal{C} \backslash A \rightarrow \mathbb{N}$. Then $(A, \tau)$ is a stopping time on $\mathcal{C}$ if

- There exists $p \in \mathbb{N}$ such that $3^{p+1}<n_{0}$, with the following property: all the connected components of $\mathcal{C} \backslash A$ are of the form $\mathcal{C}_{n_{0}, \ldots, n_{k}}$, where $n_{0}=n$, the sequence $n_{0}, \ldots, n_{k}$ is admissible, and $n_{k} \in\left[3^{p}, 3^{p+1}-1\right]$. Furthermore, $\tau$ is uniformly equal to $k$ on such a component.

- We have $\operatorname{Leb}(A) / \operatorname{Leb}(\mathcal{C}) \leqslant 1 / 2$.

Here typically $3^{p} \ll n$, thus we stop at the first occasion when the return time decreases below a certain level.

Remark 4.11. If $(A, \tau)$ is a stopping time on $\mathcal{C}$, then

$$
\frac{1}{2} \leqslant \frac{\operatorname{Leb}(\mathcal{C} \backslash A)}{\operatorname{Leb}(\mathcal{C})} \leqslant 1 .
$$

Thus in our estimates $\operatorname{Leb}(\mathcal{C} \backslash A)$ and $\operatorname{Leb}(\mathcal{C})$ may be replaced with each other. We will often use this without giving further details.

Let us define, in particular, the standard stopping time for a good curve $\mathcal{C}$ of return time $n$. Let $p$ be the integer for which $3^{p} \leqslant n^{1 / 4}<3^{p+1}$. We partition $\mathcal{C}$, iterate $T^{-1}$ and throw away the negligible parts according to the process described above. We go on iterating until either the return time of the image belongs to the interval $\left[3^{p}, 3^{p+1}-1\right]$, or the number of iterates exceeds $K \log n$. Thus we put into $A$, on the one hand, the points thrown away during this process, and, on the other hand, the intervals for which the return time does not reach $\left[3^{p}, 3^{p+1}-1\right]$ before $K \log n$ iterations. On all other intervals we define $\tau$ as the first occasion when the return time belongs to $\left[3^{p}, 3^{p+1}-1\right]$.

Proposition 4.12. The standard stopping time $(A, \tau)$ defined this way is indeed a stopping time if $n$ is large enough. Furthermore, $\operatorname{Leb}(A) / \operatorname{Leb}(\mathcal{C}) \leqslant n^{-1 / 5}$.

Proof. The only non-trivial condition to be verified is $\operatorname{Leb}(A) / \operatorname{Leb}(\mathcal{C}) \leqslant n^{-1 / 5}$.

Let us first estimate the measure of points thrown away during the refinement process. We will denote this set by $A_{0}(\subset A \subset \mathcal{C})$.

No matter which phase of the iteration we consider, the return time is $\geqslant n^{1 / 4}$, thus, according to (33), the points thrown away occupy at most a $C n^{-1 / 4}$ proportion of the considered interval. Hence, by bounded distortion, the proportion of $A_{0}$ in $\mathcal{C}$ is at most $C n^{-1 / 4} K \log n \leqslant n^{-1 / 5}$ for $n$ large enough. 
It remains to be shown that the overall measure of the intervals that do not reach $\left[3^{p}, 3^{p+1}-1\right]$ before $K \log n$ iterations is small. We have $\mathcal{C}=A_{0} \cup \cup \mathcal{C}_{i}$, where each $\mathcal{C}_{i}$ is of the form $\mathcal{C}_{n_{0}, \ldots, n_{k}}$ for some admissible sequence $n_{0}, \ldots, n_{k}$, with $k \leqslant K \log n$, and $n_{k}<3^{p+1}$ whenever $k<\lfloor K \log n\rfloor$. Thus it is enough to estimate the measure of $\mathcal{C}_{i}$-s with $\tau_{\mathcal{C}_{i}}=k=\lfloor K \log n\rfloor$. Let $\mathcal{C}^{\prime}$ be one of our standard curves of return time $n$. We apply the same construction to $\mathcal{C}^{\prime}$, and get a similar decomposition $\mathcal{C}^{\prime}=A_{0}^{\prime} \cup \cup \mathcal{C}_{i}^{\prime}$. Furthermore, by Lemma 4.9, $\frac{\operatorname{Leb} \mathcal{C}_{i}}{\operatorname{Leb} \mathcal{C}_{i}^{\prime}} \leqslant C$.

Recall that the standard curves of return time $n$ foliate the major part $M_{n}^{\prime}$ of the stripe $M_{n}$ (where $\left.\mu\left(M_{n}^{\prime}\right) / \mu\left(M_{n}\right)=1+O(1 / n)\right)$. For a fixed $i=\left(n_{0}, \ldots, n_{k}\right)$ consider $B_{i}$ the subset of the stripe $M_{n}$ that corresponds to the union of such $\mathcal{C}_{i}^{\prime}$-s for all the standard curves of return time $n$. As the density of $\mu$ on $M_{n}$ is bounded away from 0 , we get $\frac{\operatorname{Leb}\left(\mathcal{C}_{i}\right)}{\operatorname{Leb}(\mathcal{C})} \leqslant C \frac{\mu\left(B_{i}\right)}{\mu\left(M_{n}^{\prime}\right)}$. Fix $B$ as the union of all $B_{i}$-S with $\tau_{i}=\lfloor K \log n\rfloor$. When pulled back to the Young tower, the preimages of the points of $B$ are all at height at least $K \log n$. As $\pi_{X}^{*}\left(\mu_{\bar{\Delta}}\right)=\mu$, we get $\mu(B) \leqslant C \rho^{K \log n}=O\left(1 / n^{4}\right)$ by our choice of $K$. As $\mu\left(M_{n}^{\prime}\right) \sim C / n^{3}$, we may put all these estimates together to conclude that

$$
\frac{\sum_{\tau_{i}=\lfloor K \log n\rfloor} \operatorname{Leb}\left(\mathcal{C}_{i}\right)}{\operatorname{Leb}(\mathcal{C})}=O(1 / n)
$$

This completes the proof of the proposition.

In the next proposition we consider standard curves $\mathcal{C}$ and use the notation $\left(A_{\mathcal{C}}, \tau_{\mathcal{C}}\right)$ for their standard stopping times. We define a subset of the phase space, a suitable union of subcurves of standard curves, as $Y=\bigcup_{\mathcal{C}}\left(\mathcal{C} \backslash A_{\mathcal{C}}\right)$. We also consider the Birkhoff sum of $h$ with respect to $T^{-1}$ up to standard stopping time, i.e., we fix $H(x)=\sum_{k=1}^{\tau_{\mathcal{C}}(x)-1} h\left(T^{-k} x\right)$ for $x \in Y$.

Proposition 4.13. We have

$$
\int_{\bar{\Delta}} \bar{h}\left(e^{i t \bar{H}}-1\right)=\int_{Y} h\left(e^{i t H}-1\right)+O(t)
$$

This proposition plays a central role as it allows us to investigate, instead of $\int_{\bar{\Delta}} \bar{h}\left(e^{i t \bar{H}}-1\right)$ (a quantity that depends a priori on the choice of the Young tower), an expression which is much easier to handle, as it is completely explicitly given in terms of the phase space geometry.

Proof. Let us show first that

$$
\int_{\bar{\Delta}} \bar{h}\left(e^{i t \bar{H}}-1\right)=\int_{\pi_{X}^{-1}(Y)} \bar{h}\left(e^{i t \bar{H}}-1\right)+O(t) .
$$

Consider $A=X \backslash Y$. The set $A$ consists of two parts. It contains, on the one hand, the points that are not covered by standard curves and, on the other hand, those contained in $A_{\mathcal{C}}$ for some standard curve $\mathcal{C}$. These two sets will be referred to as $A_{1}$ and $A_{2}$, respectively.

We cover the set $A_{1} \cap\left\{\varphi_{-}=n\right\}$ by two further sets, the first one containing points that slide along a semi-circle (of return time $n$, this is of measure $O\left(1 / n^{4}\right)$ ), and secondly the part of $M_{n}$ not covered by standard curves, this later having measure $O\left(1 / n^{4}\right)$ as well. Altogether we have $\mu\left(A_{1} \cap\left\{\varphi_{-}=n\right\}\right)=O\left(1 / n^{4}\right)$.

According to Proposition 4.12, we have $\operatorname{Leb}\left(A_{\mathcal{C}}\right) / \operatorname{Leb}(\mathcal{C}) \leqslant n^{-1 / 5}$ whenever $n$, the return time for $\mathcal{C}$, is large enough. Integrating on the relevant standard curves we obtain $\mu\left(A_{2} \cap\left\{\varphi_{-}=n\right\}\right)=O\left(1 / n^{3+1 / 5}\right)$. 
Altogether we have

$$
\mu\left(A \cap\left\{\varphi_{-}=n\right\}\right)=O\left(1 / n^{3+1 / 5}\right) .
$$

For any $1 / p+1 / q=1$ we have

$$
\left|\int_{\pi_{X}^{-1}(A)} \bar{h}\left(e^{i t \bar{H}}-1\right)\right| \leqslant \int 1_{\pi_{X}^{-1}(A)}|\bar{h}| t|\bar{H}| \leqslant|t|\left(\int\left(1_{\pi_{X}^{-1}(A)}|\bar{h}|\right)^{p}\right)^{1 / p}\left(\int|\bar{H}|^{q}\right)^{1 / q} .
$$

Recall from Lemma 3.19 that the function $\bar{H}$ belongs to $L^{q}$ for any $q<2$, while (36) implies that $\int\left(1_{\pi_{X}^{-1}(A)}|\bar{h}|\right)^{p}$, being equal to $\int_{X} 1_{A}|h|^{p}$, is finite for $p<2+1 / 5$. We can thus take $p=2+1 / 10$ and $q=(1-1 / p)^{-1}$, to obtain (35).

Now, to complete the proof, we need to show that

$$
\int_{\pi_{X}^{-1}(Y)} \bar{h}\left(e^{i t \bar{H}}-1\right)=\int_{\pi_{X}^{-1}(Y)} \bar{h}\left(e^{i t H \circ \pi_{X}}-1\right)+O(t) .
$$

Consider $\mathcal{C}_{i}$, a connected component of $\mathcal{C} \backslash A_{\mathcal{C}}$, where $\mathcal{C}$ is a standard curve of return time $n$. Then the stopping time on $\mathcal{C}_{i}$ is an integer $\tau_{i}<K \log n$ such that $\mathcal{D}_{i}=T^{-\tau_{i}}\left(\mathcal{C}_{i}\right)$ is a good curve, with return time in the interval $\left[n^{1 / 4} / 3,3 n^{1 / 4}\right]$.

Lemma 4.14. There exists a constant $C$ such that, for any large enough integer $n$, given any good curve $\mathcal{D}$ of return time $\in\left[n^{1 / 4} / 3,3 n^{1 / 4}\right]$, the points for which the return time increases above $n^{1 / 2}$ within $K \log n$ iterations of $T^{-1}$ occupy relative measure less than $C n^{-1 / 4}$ in $\mathcal{D}$.

Proof. The map $T^{-1}$ satisfies Chernov's axioms, by Mar04. Consequently, we can use Che99, Theorem 3.1], with $\delta=Z[\mathcal{D}, \mathcal{D}, 0]^{-1 / \sigma} / n^{1 / \sigma}$. This theorem is in fact stated for LUMs, but its proof can be straightforwardly adapted to deal with manifolds close to the unstable direction.

We obtain a decreasing sequence $W_{0}^{1} \supset W_{1}^{1} \supset \cdots \supset W_{\lfloor K \log n\rfloor}^{1}$ of subsets of $\mathcal{D}$ such that, if we denote by Sing the set of singularities of $T^{-1}$,

$$
\forall c>0, \forall 0 \leqslant p \leqslant K \log n, \operatorname{Leb}\left\{x \in W_{p}^{1} \mid \operatorname{dist}\left(T^{-p} x, \operatorname{Sing}\right) \leqslant c n^{-1}\right\} \leqslant C c n^{-1}
$$

(by Equation (3.3) in [Che99]), and

$$
\forall 0 \leqslant p \leqslant K \log n, \operatorname{Leb}\left(W_{p}^{1} \backslash W_{p+1}^{1}\right) \leqslant \frac{C}{n} \operatorname{Leb}(\mathcal{D})
$$

(By (iv), (3.5) in [Che99] and our choice of $\delta$ ).

Note that the results of Che99 imply that (38) holds for the distance measured in the $p$-metric. However, we are in a region of $X$ where $\cos \theta$ is bounded away from 0 , and the stable and unstable cones are bounded away from the vertical direction by Proposition 2.1] Hence, it is equivalent to have (38) for the $p$-distance or for the usual distance.

If $T^{-p}(x)$ has a return time $\geqslant n^{1 / 2}$, then $T^{-p} x$ is at a distance at most $C n^{-1}$ of Sing. Hence, the point $x$ belongs to one of the sets whose measure is bounded in (38) and (39). This gives a measure at most $C \log n n^{-1}$. Since $\operatorname{Leb}(\mathcal{D}) \geqslant C n^{-1 / 2}$, this proves the lemma.

This lemma applies to $\mathcal{D}_{i}$. Let us write $\mathcal{C}_{i}=\mathcal{C}_{i}^{1} \cup \mathcal{C}_{i}^{2}$, where $\mathcal{C}_{i}^{2}$ corresponds to points which go to $\mathcal{D}_{i}$, and then reach a return time $>n^{1 / 2}$ in a time shorter than $K \log n$. It satisfies $\operatorname{Leb}\left(\mathcal{C}_{i}^{2}\right) / \operatorname{Leb}\left(\mathcal{C}_{i}\right) \leqslant$ $C n^{-1 / 4}$ by Lemma 4.14 . 
Let $Y_{1}=\bigcup \mathcal{C}_{i}^{1}$ and $Y_{2}=\bigcup \mathcal{C}_{i}^{2}$. Since $\mu\left(Y_{2} \cap\left\{\varphi_{-}=n\right\}\right)=O\left(1 / n^{3+1 / 4}\right)$, the proof of (35) applies and gives

$$
\int_{\pi_{X}^{-1}\left(Y_{2}\right)} \bar{h}\left(e^{i t \bar{H}}-1\right)=O(t) ; \quad \int_{\pi_{X}^{-1}\left(Y_{2}\right)} \bar{h}\left(e^{i t H \circ \pi_{X}}-1\right)=O(t) .
$$

Remark 4.15. Note that $H \circ \pi_{X}$ belongs to $L^{q}$ for any $q<2$ as it is smaller than a function to which Lemma 3.19 applies.

Hence, it is sufficient to prove (37) on $\pi_{X}^{-1}\left(Y_{1}\right)$. Let us write $\pi_{X}^{-1}\left(Y_{1}\right)=Z_{1} \cup Z_{2}$ where

$$
Z_{1}=\left\{x \in \pi_{X}^{-1}\left(Y_{1}\right) \mid \omega(x)<K \log \left(\varphi_{-}\left(\pi_{X} x\right)\right)\right\}
$$

and $Z_{2}=\pi_{X}^{-1}\left(Y_{1}\right) \backslash Z_{1}$. For $n>0$,

$$
\mu_{\bar{\Delta}}\left\{x \in Z_{2} \mid \varphi_{-}\left(\pi_{X} x\right)=n\right\} \leqslant \mu_{\bar{\Delta}}\{x \in \bar{\Delta}, \omega(x) \geqslant K \log n\}=O\left(1 / n^{4}\right) .
$$

Hence, we get once again $\int_{Z_{2}} \bar{h}\left(e^{i t \bar{H}}-1\right)=O(t)$ and $\int_{Z_{2}} \bar{h}\left(e^{i t H \circ \pi_{X}}-1\right)=O(t)$.

On $Z_{1} \cap\left\{\varphi_{-} \circ \pi_{X}=n\right\}$, the functions $\bar{H}$ and $H \circ \pi_{X}$ differ by at most $\left\|f_{0}\right\|_{\infty} K \log n n^{1 / 2}$ (corresponding to at most $K \log n$ iterations with a return time $\left.<n^{1 / 2}\right)$. Hence,

$$
\begin{aligned}
\left|\int_{Z_{1}} \bar{h}\left(e^{i t \bar{H}}-1\right)-\bar{h}\left(e^{i t H \circ \pi_{X}}-1\right)\right| & \\
& \leqslant|t| \int_{Z_{1}}|\bar{h}|\left|\bar{H}-H \circ \pi_{X}\right| \leqslant C|t| \sum_{n} \mu\left\{\varphi_{-}=n\right\} n \log n n^{1 / 2} \leqslant C|t|
\end{aligned}
$$

since $\mu\left\{\varphi_{-}=n\right\}=O\left(1 / n^{3}\right)$. This proves (37), and concludes the proof of Proposition 4.13.

\subsection{An upper bound on $H$}

The aim of this subsection is to estimate the average of the function $H$ on a good curve $\mathcal{C}$ of return time $n$. We obtain the following upper bound:

Proposition 4.16. Let $s \in[1,2)$. Consider a good curve $\mathcal{C}$ of return time $n_{0}$, and a stopping time $(A, \tau)$ on $\mathcal{C}$. Then

$$
\frac{\int_{\mathcal{C} \backslash A} \sum_{k=0}^{\tau(x)-1}\left|h\left(T^{-k} x\right)\right|^{s}}{\operatorname{Leb}(\mathcal{C} \backslash A)} \leqslant C(s) n_{0}^{s},
$$

where the constant $C(s)$ depends only on $s$.

Let us fix some notation first. There is an integer $p_{0}$ such that the return time $n_{0}$ for our good curve $\mathcal{C}$ belongs to $\left[3^{p_{0}}, 3^{p_{0}+1}-1\right]$. By the definition of stopping times, there exists another integer $p_{1}<p_{0}$ such that, for any $x \in \mathcal{C} \backslash A, \varphi_{-}\left(T^{-\tau(x)}(x)\right) \in\left[3^{p_{1}}, 3^{p_{1}+1}-1\right]$. Now consider an intermediate $p, p_{1}<p \leq p_{0}$. In the course of the proof first we investigate, in a series of lemmas, what happens while the return time descends from $\left[3^{p}, 3^{p+1}-1\right]$ to $\left[3^{p-1}, 3^{p}-1\right]$. Then we sum up for $p_{1}<p \leq p_{0}$. In the first part of the proof the value of $p$ is fixed and $n \approx 3^{p}$, while in the second part $p$ varies from $p_{1}$ to $p_{0}$. The value of $s \in[1,2)$ is fixed throughout the subsection.

According to this plan, let us fix $p \in \mathbb{N}$ large enough. Given $x \in X$, we define $\tau_{p}(x)$ as the first time $k \geqslant 1$ for which $\varphi_{-}\left(T^{-k} x\right)<3^{p}$, and $\Phi_{p}(x)=\sum_{k=0}^{\tau_{p}(x)-1}\left|\varphi_{-}\left(T^{-k} x\right)\right|^{s}$. Since $|h| \leqslant C \varphi_{-}$, it is sufficient to prove Proposition 4.16 for $h=\varphi_{-}$to conclude.

Define $R \subset X$ as the union of all standard curves with return time from the interval $\left[3^{p} / 2,3^{p}-1\right]$. 
Lemma 4.17. There exists a constant $C$ such that

$$
\int_{R} \Phi_{p} \leqslant C \mu(R) 3^{p s}
$$

Proof. Let $R_{1}=\left\{x \in R \mid \varphi_{-}\left(T^{-1} x\right)<3^{p}\right\}$ and $R_{2}=\left\{x \in R \mid \varphi_{-}\left(T^{-1} x\right) \geqslant 3^{p}\right\}$. On $R_{1}$ we have $\Phi_{p}(x)=\left|\varphi_{-}(x)\right|^{s}$, thus

$$
\int_{R_{1}} \Phi_{p} \leqslant C \mu\left(R_{1}\right) 3^{p s}
$$

Let us define $\varphi^{\prime}(x)=\varphi_{-}(x)$ for $x$ with $\varphi_{-}(x) \geqslant 3^{p-1}$ and $\varphi^{\prime}(x)=0$ otherwise. Note that $\Phi_{p}(x)=$ $\sum_{k=0}^{\tau_{p}(x)-1}\left|\varphi^{\prime}\left(T^{-k} x\right)\right|^{s}$ for $x \in R_{2}$.

Consider $Z \subset X, Z:=\left\{3^{p-1}-C_{1} \leqslant \varphi_{-}<3^{p}\right\}$, and define $\tau_{Z}: Z \rightarrow \mathbb{N}$ as the first return time to $Z$. By Kac's formula,

$$
\int_{Z}^{\tau_{Z}(x)-1} \sum_{k=0}\left|\varphi^{\prime}\left(T^{-k} x\right)\right|^{s}=\int_{X}\left|\varphi^{\prime}\right|^{s} \leqslant C \sum_{k \geqslant 3^{p-1}} \mu\left(\varphi_{-}=k\right)|k|^{s} \leqslant C \sum_{k \geqslant 3^{p-1}} \frac{1}{k^{3}} k^{s} \leqslant C \frac{3^{p s}}{3^{2 p}} .
$$

Now $R_{2} \subset Z$ and for $x \in R_{2}$ we have $\tau_{Z}(x)=\tau_{p}(x)$. Thus

$$
\int_{R_{2}} \Phi_{p}=\int_{R_{2}} \sum_{k=0}^{\tau_{Z}(x)-1}\left|\varphi^{\prime}\left(T^{-k} x\right)\right|^{s} \leqslant \int_{Z}^{\tau_{Z}(x)-1} \sum_{k=0}\left|\varphi^{\prime}\left(T^{-k} x\right)\right|^{s} .
$$

By Remark 4.8, $\frac{1}{3^{2 p}}=O\left(\mu\left(R_{2}\right)\right)$. This completes the proof.

If $\mathcal{C}$ is a good curve of return time $n \in\left[3^{p}, 3^{p+2}-1\right], \tau_{p}$ defines a stopping time on $\mathcal{C}$, with the corresponding thrown-away set that we denote by $A_{p}$. To see that it is indeed a stopping time we only need to show that $\operatorname{Leb}\left(A_{p}\right) \leqslant \operatorname{Leb}(\mathcal{C}) / 2$. Now consider the standard stopping time $\tau_{\mathcal{C}}$ with its thrown away set $A_{\mathcal{C}}$. Then $A_{p} \subset A_{\mathcal{C}}$ while $\operatorname{Leb}\left(A_{\mathcal{C}}\right) \leqslant n^{-1 / 5} \operatorname{Leb}(\mathcal{C})$ by Proposition 4.12, which gives the claim.

The first step in the proof of Proposition [4.16 is the estimate

$$
\frac{\int_{\mathcal{C} \backslash A_{p}} \Phi_{p}}{\operatorname{Leb}\left(\mathcal{C} \backslash A_{p}\right)} \leqslant C 3^{p s}
$$

for a good curve $\mathcal{C}$ with return time $n \in\left[3^{p}, 3^{p+1}-1\right]$. To show this, we will relate the average of $\Phi_{p}$ on $\mathcal{C}$ to its average on $R$.

Consider $B=\bigcup\left(\mathcal{C} \backslash A_{p}\right)$, where the union is taken over all standard curves of return time from the interval $\left[3^{p}, 3^{p+2}-1\right]$.

Lemma 4.18. There is a constant $C$ such that, for any good curve $\mathcal{C}$ of return time $n \in\left[3^{p}, 3^{p+1}-1\right]$,

$$
\frac{\int_{\mathcal{C} \backslash A_{p}} \Phi_{p}}{\operatorname{Leb}\left(\mathcal{C} \backslash A_{p}\right)} \leqslant C \frac{\int_{B} \Phi_{p}}{\mu(B)}+C 3^{p s}
$$


Proof. Let $U=\left\{x \in \mathcal{C} \mid \varphi_{-}\left(T^{-1} x\right) \geqslant 3^{p}\right\}$. On $\mathcal{C}$, we have $\Phi_{p}(x)=\left|\varphi_{-}(x)\right|^{s}+1_{U}(x) \Phi_{p}\left(T^{-1} x\right)$. To prove (41), it is enough to show

$$
\int_{U \cap\left(\mathcal{C} \backslash A_{p}\right)} \Phi_{p} \circ T^{-1} \leqslant C \frac{\int_{B} \Phi_{p}}{\mu(B)} \operatorname{Leb}\left(\mathcal{C} \backslash A_{p}\right) .
$$

By bounded distortion, this can be further reduced to

$$
\frac{\int_{T^{-1}\left(\mathcal{C} \backslash A_{p}\right) \cap\left\{\varphi_{-} \geqslant 3^{p}\right\}} \Phi_{p}}{\operatorname{Leb}\left(T^{-1}\left(\mathcal{C} \backslash A_{p}\right) \cap\left\{\varphi_{-} \geqslant 3^{p}\right\}\right)} \leqslant C \frac{\int_{B} \Phi_{p}}{\mu(B)} .
$$

Let $q$ be the maximal possible return time the points of $T^{-1}\left(\mathcal{C} \backslash A_{p}\right)$ have. It satisfies $3^{p+2}>q \geqslant$ $3^{p+1}-C_{2}$. By Lemma 4.9

$$
\frac{\int_{T^{-1}\left(\mathcal{C} \backslash A_{p}\right) \cap\left\{\varphi_{-} \geqslant 3^{p}\right\}} \Phi_{p}}{\operatorname{Leb}\left(T^{-1}\left(\mathcal{C} \backslash A_{p}\right) \cap\left\{\varphi_{-} \geqslant 3^{p}\right\}\right)} \leqslant C \frac{\int_{B \cap\left\{3^{p} \leqslant \varphi_{-} \leqslant q\right\}} \Phi_{p}}{\mu\left(B \cap\left\{3^{p} \leqslant \varphi_{-} \leqslant q\right\}\right)} .
$$

As $q \geqslant 3^{p+1}-C_{2}$, by Remark $4.8 \mu(B) \leqslant C \mu\left(B \cap\left\{3^{p} \leqslant \varphi_{-} \leqslant q\right\}\right)$. This implies (42) and completes the proof.

Now $B$ is not exactly $R$, we need to "widen up" the estimate of Lemma 4.17 from $R$ to $B$ to obtain (40).

Let $B_{1}=B \cap\left\{3^{p} \leqslant \varphi_{-}<3^{p+1} / 2\right\}, B_{2}=B \cap\left\{3^{p+1} / 2 \leqslant \varphi_{-}<3^{p+1}\right\}, B_{3}=B \cap\left\{3^{p+1} \leqslant \varphi_{-}<3^{p+2} / 2\right\}$ and $B_{4}=B \cap\left\{3^{p+2} / 2 \leqslant \varphi_{-}<3^{p+2}\right\}$.

Lemma 4.19. There exists a constant $C$ such that, for any good curve $\mathcal{C}$ of return time $n \in$ $\left[3^{p}, 3^{p+1} / 2\right)$,

$$
\frac{\int_{B_{1}} \Phi_{p}}{\mu\left(B_{1}\right)} \leqslant C \frac{\int_{\mathcal{C} \backslash A_{p}} \Phi_{p}}{\operatorname{Leb}\left(\mathcal{C} \backslash A_{p}\right)}
$$

Proof. The curve $T^{-1}(\mathcal{C})$ crosses all stripes of return time between $3^{p}$ and $3^{p+1} / 2$. This allows us to apply the argument of Lemma 4.18 with reversed inequalities.

Lemma 4.20. There exists a constant $C$ such that

$$
\frac{\int_{B_{1}} \Phi_{p}}{\mu\left(B_{1}\right)} \leqslant C 3^{p s}
$$

Proof. Let $\mathcal{C}$ be a standard curve of return time $n \in\left[3^{p} / 2,3^{p}-1\right]$. For $i \in\left[3^{p}, 3^{p+1} / 2\right)$, put $\mathcal{C}_{i}=$ $\left\{x \in \mathcal{C}, \varphi_{-}\left(T^{-1} x\right)=i\right\}$ and let $\mathcal{D}_{i}$ be its image by $T^{-1}$. This is a good curve of return time $i$ and, by bounded distortion,

$$
\frac{\int_{\mathcal{D}_{i}} \Phi_{p}}{\operatorname{Leb}\left(\mathcal{D}_{i}\right)} \leqslant C \frac{\int_{\mathcal{C}_{i}} \Phi_{p}}{\operatorname{Leb}\left(\mathcal{C}_{i}\right)}
$$

Furthermore, applying Lemma 4.19 to $\mathcal{D}_{i}$, we get

$$
\operatorname{Leb}\left(\mathcal{C}_{i}\right) \frac{\int_{B_{1}} \Phi_{p}}{\mu\left(B_{1}\right)} \leqslant C \int_{\mathcal{C}_{i}} \Phi_{p}
$$


As by Remark 4.8 the good curves $\mathcal{C}_{i}$ occupy a fixed proportion of $\mathcal{C}$, we may sum up

$$
\operatorname{Leb}(\mathcal{C}) \frac{\int_{B_{1}} \Phi_{p}}{\mu\left(B_{1}\right)} \leqslant C \int_{\mathcal{C}} \Phi_{p}
$$

Integrating over all standard curves of return time $\in\left[3^{p} / 2,3^{p}-1\right]$, we obtain

$$
\mu(R) \frac{\int_{B_{1}} \Phi_{p}}{\mu\left(B_{1}\right)} \leqslant C \int_{R} \Phi_{p} .
$$

We may conclude by Lemma 4.17 .

Lemma 4.21. There is a constant $C$ such that for any $l=2,3,4$,

$$
\frac{\int_{B_{l}} \Phi_{p}}{\mu\left(B_{l}\right)} \leqslant C 3^{p s} .
$$

Proof. As the three cases are essentially identical we give the argument only for one of them, for $l=3$, say. The proof is analogous to that of the previous lemma, we only need to apply a bit more iterations. Let $\mathcal{C}$ be a standard curve with return time from $\left[3^{p} / 2,3^{p}-1\right]$. Given $i \in\left[3^{p}, 3^{p+1} / 2\right)$, let $\mathcal{C}_{i}$ be the set of points in $\mathcal{C}$ the images of which have return time $i$. For $j \in\left[3^{p+1} / 2,3^{p+1}\right)$, let $\mathcal{C}_{i j}$ be the set of points in $\mathcal{C}_{i}$ the $T^{-2}$-images of which have return time $j$. Finally, for $k \in\left[3^{p+1}, 3^{p+2} / 2\right)$, we define $\mathcal{C}_{i j k}$ analogously.

By Remark 4.8, at each step we keep a fixed proportion of the previous set. Thus, there exists a constant $C$ such that

$$
\operatorname{Leb}(\mathcal{C}) \leqslant C \sum_{i, j, k} \operatorname{Leb}\left(\mathcal{C}_{i j k}\right)
$$

Following the lines of the proof of Lemma 4.19 we may show that given any good curve $\mathcal{D}$ of return time from the interval $\left[3^{p+1}, 3^{p+2} / 2\right)$, we have $\frac{\int_{B_{3}} \Phi_{p}}{\mu\left(B_{3}\right)} \leqslant C \frac{\int_{\mathcal{D}} \Phi_{p}}{\operatorname{Leb}(\mathcal{D})}$. This applies, in particular, to $\mathcal{D}=T^{-3}\left(\mathcal{C}_{i j k}\right)$ and gives

$$
\frac{\int_{B_{3}} \Phi_{p}}{\mu\left(B_{3}\right)} \leqslant C \frac{\int_{T^{-3} \mathcal{C}_{i j k}} \Phi_{p}}{\operatorname{Leb}\left(T^{-3} \mathcal{C}_{i j k}\right)} \leqslant C \frac{\int_{\mathcal{C}_{i j k}} \Phi_{p}}{\operatorname{Leb}\left(\mathcal{C}_{i j k}\right)},
$$

by bounded distortion. We may apply Lemma 4.17, just as we did in the proof of Lemma 4.20, to get the desired conclusion.

Lemmas 4.20, 4.21 and 4.18 altogether imply the bound (40) for any good curve of return time $n \in$ $\left[3^{p}, 3^{p+1}-1\right]$. We apply this bound in the second (much easier) step of the proof of Proposition 4.16.

Proof of Proposition 4.16, Recall the notations from the beginning of the subsection: $\mathcal{C}$ is a good curve of return time $n_{0} \in\left[3^{p_{0}}, 3^{p_{0}+1}-1\right]$, for some large $p_{0}$, and the stopping time $\tau$ is related to another integer $p_{1}\left(p_{0}>p_{1}\right): \varphi_{-}\left(T^{-\tau(x)}(x)\right) \in\left[3^{p_{1}}, 3^{p_{1}+1}-1\right]$ for all $x \in \mathcal{C} \backslash A$.

To simplify notation in this proof we define $\tau_{p_{0}+1}(x)=0$ for $x \in \mathcal{C} \backslash A$. For $x \in \mathcal{C} \backslash A$ we have

$$
\sum_{k=0}^{\tau(x)-1}\left|\varphi_{-}\left(T^{-k} x\right)\right|^{s}=\sum_{p=p_{1}+1}^{p_{0}} \Phi_{p}\left(T^{-\tau_{p+1}(x)} x\right) .
$$


Let $p_{1}+1 \leqslant p \leqslant p_{0}$ and $x \in \mathcal{C} \backslash A$. Then there is a subcurve $\mathcal{C}_{i} \subset \mathcal{C}$ that contains $x$ and for which $T^{-\tau_{p+1}(x)}\left(\mathcal{C}_{i}\right)$, to be denoted by $\mathcal{D}_{i}$, is a good curve of return time from $\left[3^{p}, 3^{p+1}-1\right]$. By bounded distortion

$$
\frac{\int_{\mathcal{C}_{i} \backslash A} \Phi_{p}\left(T^{-\tau_{p+1}} y\right)}{\operatorname{Leb}\left(\mathcal{C}_{i}\right)} \leqslant C \frac{\int_{T^{-\tau_{p+1}}\left(\mathcal{C}_{i} \backslash A\right)} \Phi_{p}}{\operatorname{Leb}\left(\mathcal{D}_{i}\right)} \leqslant C \frac{\int_{\mathcal{D}_{i} \backslash A_{p}} \Phi_{p}}{\operatorname{Leb}\left(\mathcal{D}_{i}\right)} .
$$

Now according to (40) this final quantity is bounded from above by $C 3^{p s}$. Summing up for all intervals $\mathcal{C}_{i}$ we obtain

$$
\int_{\mathcal{C} \backslash A} \Phi_{p}\left(T^{-\tau_{p+1}(y)} y\right) \leqslant C 3^{p s} \operatorname{Leb}(\mathcal{C}) .
$$

Summation on $p$ from $p_{1}+1$ to $p_{0}$ implies the statement.

Corollary 4.22. We have

$$
\int_{Y} h\left(e^{i t H}-1\right)=i t \int_{Y} h 1_{\varphi_{-} \leqslant 1 /|t|} H+o(t \log (1 /|t|)) .
$$

Proof. We have

$$
\left|\int_{Y} h 1_{\varphi_{-} \leqslant 1 /|t|}\left(e^{i t H}-1-i t H\right)\right| \leqslant C \int_{Y}|h| 1_{\varphi_{-} \leqslant 1 /|t|}|t|^{3 / 2}|H|^{3 / 2} .
$$

We may estimate $|H(x)|^{3 / 2}$ as

$$
|H(x)|^{3 / 2} \leqslant\left(\sum_{1}^{\tau(x)-1}\left|h\left(T^{-k} x\right)\right|\right)^{3 / 2} \leqslant \tau(x)^{1 / 2} \sum_{0}^{\tau(x)-1}\left|h\left(T^{-k} x\right)\right|^{3 / 2} .
$$

Now put $\Phi(x)=\sum_{0}^{\tau(x)-1}\left|h\left(T^{-k} x\right)\right|^{3 / 2}$. Then for $x \in Y$ of return time $n$ we get $|H(x)|^{3 / 2} \leqslant$ $(K \log n)^{1 / 2} \Phi(x)$, as the standard stopping time satisfies $\tau(x) \leqslant K \log n$.

By Proposition 4.16 the average of the function $\Phi$ on $Y \cap\left\{\varphi_{-}=n\right\}$ is less than $c n^{3 / 2}$. Putting these estimates together

$$
\begin{aligned}
\int_{Y}|h| 1_{\varphi_{-} \leqslant 1 /|t|}|t|^{3 / 2}|H|^{3 / 2} & \leqslant C|t|^{3 / 2} \sum_{n=1}^{1 /|t|} \mu\left(\varphi_{-}=n\right) \sqrt{\log n} n n^{3 / 2} \\
& \leqslant C|t|^{3 / 2} \sqrt{\log (1 /|t|)}|t|^{-1 / 2}=o(|t| \log (1 /|t|)),
\end{aligned}
$$

while

$$
\left|\int_{Y} h 1_{\varphi_{-}>1 /|t|}\left(e^{i t H}-1\right)\right| \leqslant C \int \varphi_{-} 1_{\varphi_{-}>1 /|t|} \leqslant C \sum_{n>1 /|t|} \mu\left(\varphi_{-}=n\right) n=O(t)
$$

as $\mu\left(\varphi_{-}=n\right)=O\left(1 / n^{3}\right)$

\subsection{Exact asymptotics for $H$}

Recall the value of $I$ from (1i), and the fact that on $Y \cap\left\{\varphi_{-}=n\right\}$ the function $h$ is equivalent to $n I$. 
Lemma 4.23. Let $y=\frac{1}{1-\frac{3}{4} \log 3}$. For any $\varepsilon>0$ there exists $N_{0} \in \mathbb{N}$ such that, for all $n \geqslant N_{0}$, for all good curve $\mathcal{C}$ with return time $n$,

$$
\left|\frac{\int_{\mathcal{C} \backslash A_{\mathcal{C}}} H}{\operatorname{Leb}\left(\mathcal{C} \backslash A_{\mathcal{C}}\right)}-n(y-1) I\right| \leqslant \varepsilon n
$$

Proof. Recall the asymptotic expressions for the transition probabilities from Remark 4.8. These allow us to regard the map $T^{-1}$ as a Markov chain. Then the statement of the lemma can be guessed by the expectation value with respect to the invariant distribution of this chain.

The rigorous proof is inductive. Note that first we fix $\varepsilon>0$, that will correspond to the required precision in the asymptotics, and then we may choose $n$ arbitrarily large. Let $L \in \mathbb{N}$ be an integer for which $(9 / 10)^{L} \leqslant \varepsilon$. This integer $L$ is the number of inductive steps needed to obtain $\varepsilon$-precision. More precisely, if $n_{0}, \ldots, n_{L}$ is an admissible sequence (here $n_{0}=n$ ), then $n_{L}$ is typically much smaller than $n_{0}$. The Birkhoff sum of $h$ for the times between $n_{L}$ and the stopping time can be estimated by the upper bound coming from Lemma 4.16, which roughly means that we only need to take care of the sum for the first $L$ steps. This estimate will be the starting point of our induction. Then we place our standard curve "high enough" (i.e., choose $n$ large enough) to ensure that the transition probabilities of Remark 4.8 are accurate with very good precision. These transition probabilities are responsible for the appearance of $y$ as we decrease the length of the admissible sequence $n_{0}, \ldots, n_{i}$ from $i=L$ to $i=0$ in the induction.

Let $\mathcal{C}$ be a standard curve of return time $n$ with the standard stopping time $\left(A_{\mathcal{C}}, \tau_{\mathcal{C}}\right)$ on it. If $n_{0}, \ldots, n_{i}$ is admissible with $n_{0}=n$ and $i \leqslant L$, the set $\mathcal{C}_{n_{0}, \ldots, n_{i}}$ is not empty, and we may consider $\mathcal{C}_{n_{0}, \ldots, n_{i}}^{\prime}=$ $\mathcal{C}_{n_{0}, \ldots, n_{i}} \cap\left(\mathcal{C} \backslash A_{\mathcal{C}}\right)$. For $\mathcal{D}=T^{-i}\left(\mathcal{C}_{n_{0}, \ldots, n_{i}}\right)$, define $A=\mathcal{D} \backslash T^{-i}\left(\mathcal{C}_{n_{0}, \ldots, n_{i}}^{\prime}\right)$ and $\tau\left(T^{-i} x\right)=\tau_{\mathcal{C}}(x)-i$. Then, for large enough $n,(A, \tau)$ is a stopping time on $\mathcal{D}$. To see this we note that $\operatorname{Leb}(A) \leqslant \operatorname{Leb}(\mathcal{D}) / 2$ as the number of iterations is bounded from above by $L$ while $\operatorname{Leb}\left(A_{\mathcal{C}}\right) / \operatorname{Leb}(\mathcal{C}) \rightarrow 0$ as $n \rightarrow+\infty$.

By increasing $n$ if necessary, we may assume that for any $p>n / 3^{L}$, and for any $x$ with $\varphi_{-}(x)=p$ we have $|h(x)-p I| \leqslant p /\left(L 3^{L}\right)$.

Thus for $x \in \mathcal{C}_{n_{0}, \ldots, n_{L}}^{\prime}$ we have

$$
\left|H(x)-\left(n_{1}+\cdots+n_{L-1}\right) I\right| \leqslant \sum_{k=1}^{L-1}\left|h\left(T^{-k} x\right)-n_{k} I\right|+\sum_{k=L}^{\tau_{\mathcal{C}}(x)-1} \mid h\left(T^{-k}(x) \mid\right.
$$

where the first term satisfies

$$
\sum_{k=1}^{L-1}\left|h\left(T^{-k} x\right)-n_{k} I\right| \leqslant \sum_{k=1}^{L-1} n_{k} /\left(L 3^{L}\right) \leqslant n_{L}
$$

as $n_{k} \leqslant 3^{L} n_{L}$. On the other hand if we integrate the second term in (43), we may use the upper bound of Lemma 4.16. We get, for some constant $C_{3}$ :

$$
\left|\frac{\int_{\mathcal{C}_{n_{0}, \ldots, n_{L}}^{\prime}} H-\left(n_{1}+\cdots+n_{L-1}\right) I}{\operatorname{Leb}\left(\mathcal{C}_{n_{0}, \ldots, n_{L}}^{\prime}\right)}\right| \leqslant C_{3} n_{L}
$$

Choose $n$ large enough to ensure that (i) all the $\varepsilon_{p}$ from Remark 4.8 are less than $\varepsilon$ whenever $p>n / 3^{L}$, and that (ii) the distortion of any $\left.T^{-i}\right|_{\mathcal{C}_{n_{0}, \ldots, n_{i}}}, i \leq L$ is bounded from above by $\varepsilon$. 
As $y \frac{3}{4} \log 3-y+1=0$ we have, for $n$ large enough,

$$
\left|y \sum_{p / 3+C_{2}}^{3 p-C_{2}} \frac{3}{8 k}-y+1\right|<\varepsilon
$$

whenever $p>n / 3^{L}$.

To simplify notation we introduce $\alpha=\frac{9}{10}$ and another positive number, $\beta>2 \log 3$ which is, however, not too big so that $\frac{3}{8} \beta<\alpha$. By further increasing $n$, if necessary, we may also assume that $\sum_{p / 3}^{3 p} \frac{1}{k} \leqslant \beta$ whenever $p>n / 3^{L}$.

Now, by induction on decreasing $i$ we show the following bound:

$$
\left|\frac{\int_{\mathcal{C}_{n_{0}, \ldots, n_{i}}^{\prime}} H-\left(n_{1}+\cdots+n_{i-1}\right) I}{n_{i} \operatorname{Leb}\left(\mathcal{C}_{n_{0}, \ldots, n_{i}}^{\prime}\right)}-y I\right| \leqslant \alpha^{L-i}\left(C_{3}+y|I|\right)+C_{4} \sum_{k=i}^{L-1} \varepsilon \alpha^{k-i},
$$

where $C_{4}$ is some constant. Note that for $i=0$, when the sum $n_{1}+\cdots+n_{i-1}$ is to be interpreted as $-n_{0}$, this bound implies the statement of Lemma 4.23. On the other hand, the case $i=L$ is already established in (44). So let us assume (46) holds for $i$, and show it for $i-1$. We have

$$
\begin{aligned}
& \frac{\int_{\mathcal{C}_{n_{0}, \ldots, n_{i-1}}^{\prime}} H-(}{\left.n_{1}+\cdots+n_{i-2}\right) I}-y I \\
&=\frac{\int_{\mathcal{C}_{n_{0}, \ldots, n_{i-1}}^{\prime} \operatorname{Leb}\left(\mathcal{C}_{n_{0}, \ldots, n_{i-1}}^{\prime}\right)} H-\left(n_{1}+\cdots+n_{i-1}\right) I}{n_{i-1} \operatorname{Leb}\left(\mathcal{C}_{n_{0}, \ldots, n_{i-1}}^{\prime}\right)}-y I+I \\
&=\sum_{n_{i}=n_{i-1} / 3+C_{2}}^{3 n_{i-1}-C_{2}} \frac{\int_{\mathcal{C}_{n_{0}, \ldots, n_{i}}^{\prime}} H-\left(n_{1}+\cdots+n_{i-1}\right) I}{n_{i-1} \operatorname{Leb}\left(\mathcal{C}_{n_{0}, \ldots, n_{i-1}}^{\prime}\right)}-y I+I \\
&=\sum_{n_{i}=n_{i-1} / 3+C_{2}}^{3 n_{i-1}-C_{2}}\left(\frac{\int_{\mathcal{C}_{n_{0}, \ldots, n_{i}}^{\prime}} H-\left(n_{1}+\cdots+n_{i-1}\right) I}{n_{i} \operatorname{Leb}\left(\mathcal{C}_{n_{0}, \ldots, n_{i}}^{\prime}\right)} \frac{n_{i} \operatorname{Leb}\left(\mathcal{C}_{n_{0}, \ldots, n_{i}}^{\prime}\right)}{n_{i-1} \operatorname{Leb}\left(\mathcal{C}_{n_{0}, \ldots, n_{i-1}}^{\prime}\right)}-\frac{3}{8 n_{i}} y I\right) \\
&+\left(y \sum_{n_{i}=n_{i-1} / 3+C_{2}}^{3 n_{i-1}-C_{2}} \frac{3}{8 n_{i}}-y+1\right) I .
\end{aligned}
$$

The choice of a large enough $n$ ensures that even $n_{i}$ is large enough so that (45) applies:

$$
\left|y \sum_{n_{i}=n_{i-1} / 3+C_{2}}^{3 n_{i-1}-C_{2}} \frac{3}{8 n_{i}}-y+1\right| \leqslant \varepsilon
$$

Now we will use the transition probabilities (34) on the curve $T^{-(i-1)}\left(\mathcal{C}_{n_{0}, \ldots, n_{i-1}}\right)$. We will also use that the distortions of $T^{-(i-1)}$, when restricted to this curve, are bounded from above by $\varepsilon$. Note furthermore that $\mathcal{C}_{n_{0}, \ldots, n_{i-1}}^{\prime}$ occupies at least $(1-\varepsilon)$-proportion of $\mathcal{C}_{n_{0}, \ldots, n_{i-1}}$ if $n$ is large enough (we may apply Proposition 4.12). The same holds for $\mathcal{C}_{n_{0}, \ldots, n_{i}}^{\prime}$ in $\mathcal{C}_{n_{0}, \ldots, n_{i}}$. These observations allow us to obtain (note $n_{i-1} / n_{i} \leq 3$ ):

$$
\left|\frac{\operatorname{Leb}\left(\mathcal{C}_{n_{0}, \ldots, n_{i}}\right)}{\operatorname{Leb}\left(\mathcal{C}_{n_{0}, \ldots, n_{i-1}}\right)}-\frac{\operatorname{Leb}\left(\mathcal{C}_{n_{0}, \ldots, n_{i}}^{\prime}\right)}{\operatorname{Leb}\left(\mathcal{C}_{n_{0}, \ldots, n_{i-1}}^{\prime}\right)}\right| \leqslant 2 \varepsilon \frac{\operatorname{Leb}\left(\mathcal{C}_{n_{0}, \ldots, n_{i}}\right)}{\operatorname{Leb}\left(\mathcal{C}_{n_{0}, \ldots, n_{i-1}}\right)} \leqslant \frac{C \varepsilon}{n_{i}}
$$


One more reference to Remark 4.8 and to the fact that the distortions can be made smaller than $\varepsilon$ if $n$ is large enough implies

$$
\left|\frac{n_{i} \operatorname{Leb}\left(\mathcal{C}_{n_{0}, \ldots, n_{i}}^{\prime}\right)}{n_{i-1} \operatorname{Leb}\left(\mathcal{C}_{n_{0}, \ldots, n_{i-1}}^{\prime}\right)}-\frac{3}{8 n_{i}}\right| \leqslant \frac{C_{5} \varepsilon}{n_{i}}
$$

By the triangular inequality,

$$
\begin{aligned}
\mid \frac{\int_{\mathcal{C}_{n_{0}, \ldots, n_{i}}^{\prime}} H-\left(n_{1}+\cdots+n_{i-1}\right) I}{n_{i} \operatorname{Leb}\left(\mathcal{C}_{n_{0}, \ldots, n_{i}}^{\prime}\right)} & \frac{n_{i} \operatorname{Leb}\left(\mathcal{C}_{n_{0}, \ldots, n_{i}}^{\prime}\right)}{n_{i-1} \operatorname{Leb}\left(\mathcal{C}_{n_{0}, \ldots, n_{i-1}}^{\prime}\right)}-\frac{3}{8 n_{i}} y I \mid \\
& \leqslant\left|\frac{\int_{\mathcal{C}_{n_{0}, \ldots, n_{i}}^{\prime}} H-\left(n_{1}+\cdots+n_{i-1}\right) I}{n_{i} \operatorname{Leb}\left(\mathcal{C}_{n_{0}, \ldots, n_{i}}^{\prime}\right)}-y I\right| \frac{3}{8 n_{i}}|| \frac{n_{i} \operatorname{Leb}\left(\mathcal{C}_{n_{0}, \ldots, n_{i}}^{\prime}\right)}{n_{i-1} \operatorname{Leb}\left(\mathcal{C}_{n_{0}, \ldots, n_{i-1}}^{\prime}\right)}-\frac{3}{8 n_{i}} \mid .
\end{aligned}
$$

Let $B_{i}$ be the bound at step $i$ of the induction. Then the first term is bounded from above by $\frac{3 B_{i}}{8 n_{i}}$, and the second term is bounded from above by $\frac{\left(B_{i}+y|I|\right) C_{5} \varepsilon}{n_{i}}$.

Recall the definitions of $\alpha$ and $\beta$, we have $\sum_{p / 3}^{3 p} \frac{1}{k} \leqslant \beta$ and, if $\varepsilon$ is small enough, $\left(\frac{3}{8}+C_{5} \varepsilon\right) \beta<\alpha$.

Putting our estimates together we get

$$
\begin{aligned}
B_{i-1} & =\varepsilon|I|+\sum_{n_{i-1} / 3+C_{2}}^{3 n_{i}-C_{2}}\left[\frac{3 B_{i}}{8 n_{i}}+\frac{\left(B_{i}+y|I|\right) C_{5} \varepsilon}{n_{i}}\right] \leqslant \varepsilon|I|+\left(\frac{3 B_{i}}{8}+\left(B_{i}+y|I|\right) C_{5} \varepsilon\right) \beta \\
& \leqslant\left(|I|+C_{5} y|I| \beta\right) \varepsilon+\alpha B_{i},
\end{aligned}
$$

Now if (46) holds for $i$ with $C_{4}=|I|+C_{5} y|I| \beta$, it holds for $i-1$ with the same constants.

Taking $i=0$ we get

$$
\frac{1}{n}\left|\frac{\int_{\mathcal{C} \backslash A_{\mathcal{C}}} H}{\operatorname{Leb}\left(\mathcal{C} \backslash A_{\mathcal{C}}\right)}-n(y-1) I\right| \leqslant C \alpha^{L}+C \varepsilon \leqslant C \varepsilon
$$

by the choice of $L$. Note that the constant $C$ depends only on $I$, thus it can be "swallowed" by $\varepsilon$. This completes the proof of the lemma.

Proposition 4.24. We have

$$
\int_{Y} h 1_{\varphi_{-} \leqslant 1 /|t|} H=\left(\frac{I^{2}(y-1) \ell^{2}}{\pi}+o(1)\right) \log (1 /|t|) .
$$

Proof. First let us show that

$$
\int_{Y}\left(h-\varphi_{-} I\right) 1_{\varphi_{-} \leqslant 1 /|t|} H=o(\log (1 /|t|))
$$

Fix $\varepsilon>0$. If $N$ is large enough we have $\left|h-\varphi_{-} I\right| \leqslant \varepsilon \varphi_{-}$for $\varphi_{-} \geqslant N$. Thus we get (note that $H$ is integrable, cf. Remark 4.15)

$$
\left|\int_{Y}\left(h-\varphi_{-} I\right) 1_{\varphi_{-} \leqslant 1 /|t|} H\right| \leqslant O(1)+\sum_{N \leqslant n \leqslant 1 /|t|} \varepsilon n \int_{Y \cap\left\{\varphi_{-}=n\right\}}|H| .
$$


We may apply Proposition 4.16 with $s=1$ to show $\int_{Y \cap\left\{\varphi_{-}=n\right\}}|H| \leqslant C n \mu\left(\varphi_{-}=n\right)=O\left(1 / n^{2}\right)$. Thus we get

$$
\left|\int_{Y}\left(h-\varphi_{-} I\right) 1_{\varphi_{-} \leqslant 1 /|t|} H\right| \leqslant O(1)+C \varepsilon \log (1 /|t|) \leqslant C^{\prime} \varepsilon \log (1 /|t|) .
$$

As the above inequality is true for any fixed $\varepsilon>0$, we get (47).

Now we estimate

$$
\int_{Y} \varphi_{-} I 1_{\varphi_{-} \leqslant 1 /|t|} H=\sum_{n=1}^{1 /|t|} n I \int_{Y \cap\left\{\varphi_{-}=n\right\}} H .
$$

By Lemma 4.23 we have $\int_{Y \cap\left\{\varphi_{-}=n\right\}} H \sim(y-1) \operatorname{In} \mu\left(\varphi_{-}=n\right) \sim(y-1) \operatorname{In} \frac{\ell^{2}}{\pi n^{3}}$. Actually, the measure of the set $\left\{\varphi_{-}=n\right\}$ can be estimated by direct geometric arguments. Up to negligible terms, it is equivalent to $\frac{\ell^{2}}{4 \pi n^{3}}$ in all relevant zones of $X$ which are "corners of parallelograms". As there are 4 such relevant zones we obtain the above formula.

Finally we get

$$
\int_{Y} \varphi_{-} I 1_{\varphi_{-} \leqslant 1 /|t|} H \sim \sum_{n=1}^{1 /|t|} n I^{2}(y-1) n \frac{\ell^{2}}{\pi n^{3}} \sim \frac{I^{2}(y-1) \ell^{2}}{\pi} \log (1 /|t|),
$$

which completes the proof.

Proposition 4.1 follows from the combination of Proposition 4.24, Corollary 4.22, Proposition 4.13 and Lemmas 4.3, 4.2.

\section{Proof of the main theorems}

In this section, we prove Theorems 1.1 and 1.3. The main tool will be an abstract theorem showing that, if an induced map satisfies a limit theorem, then the original map satisfies the same limit theorem. Such a result has been proved in the case of flows by [MT04, and extended to the discrete time case (and to non-polynomial normalizations) in Gou03]. For the convenience of the reader, we state here the result we will use.

If $Y$ is a subset of a probability space $(X, m), T: X \rightarrow X$, and $T_{Y}$ is the induced map on $Y$, we will write $S_{n}^{Y} g=\sum_{k=0}^{n-1} g \circ T_{Y}^{k}$ : this is the Birkhoff sum of $g$, for the transformation $T_{Y}$. We will also write $E_{Y}(g)=\frac{\int_{Y} g}{m[Y]}$. Finally, for $t \in \mathbb{R},\lfloor t\rfloor$ denotes the integer part of $t$.

Theorem 5.1. Let $T: X \rightarrow X$ be an ergodic endomorphism of a probability space $(X, m)$, and $f: X \rightarrow \mathbb{R}$ an integrable function with vanishing integral. Let $Y \subset X$ have positive measure. For $y \in Y$, write $\varphi(y)=\inf \left\{n>0 \mid T^{n}(y) \in Y\right\}$ and $f_{Y}(y)=\sum_{k=0}^{\varphi(y)-1} f\left(T^{k} y\right)$.

We assume the following properties:

1. There exists a sequence $B_{n} \rightarrow+\infty$, with $\inf _{r \geqslant n} \frac{B_{r}}{B_{n}}>0$, such that $f_{Y}$ satisfies a limit theorem for the normalization $B_{n}$ : there exists a random variable $Z$ such that, for every $t \in \mathbb{R}$,

$$
E_{Y}\left(e^{i t \frac{S_{\lfloor n m(Y)\rfloor}^{Y} f_{Y}}{B n}}\right) \rightarrow E\left(e^{i t Z}\right)
$$


2. There exists $b>0$ such that, in the natural extension of $T_{Y}, \frac{1}{N^{b}} \sum_{0}^{N-1} f_{Y}\left(T_{Y}^{k} y\right)$ tends almost everywhere to 0 when $N \rightarrow \pm \infty$.

3. There exists $B_{n}^{\prime}=O\left(B_{n}^{1 / b}\right)$ such that $\frac{S_{n}^{Y} \varphi-n E_{Y}(\varphi)}{B_{n}^{\prime}}$ converges in distribution.

Then the function $f$ satisfies also a limit theorem:

$$
E\left(e^{i t \frac{S_{n} f}{B_{n}}}\right) \rightarrow E\left(e^{i t Z}\right)
$$

i.e., $\frac{S_{n} f}{B_{n}}$ tends in distribution to $Z$.

The first assumption is apparently different from the first assumption in [Gou03, Theorem A.1]. However, they are equivalent by Eag76 (see also [MT04]).

Remark 5.2. An analogous theorem holds in the case of flows, when $Y$ is a Poincaré section of the flow and $\varphi$ is the return time to this Poincaré section, with the same proof. Since a Poincaré section has usually zero measure, it has to be formulated slightly differently: $E_{Y}$ will be the expectation with respect to the probability measure induced by $m$ on $Y$, and in (48) $m(Y)$ should be replaced with $1 / E_{Y}(\varphi)$. Finally, the sums (in the definition of $f_{Y}$, and in the definition of the Birkhoff sums of $f$ ) should be replaced with integrals, and correspondingly, the normalizing sequences $B_{n}\left(B_{n}^{\prime}\right)$ with appropriate functions $B(T)\left(B^{\prime}(T)\right), B: \mathbb{R}^{+} \rightarrow \mathbb{R}^{+}$.

\subsection{Proof of Theorem 1.1 for functions satisfying $(P 1)$}

Let $f_{0}: X_{0} \rightarrow \mathbb{R}$ be Hölder continuous and satisfy $(P 1)$. In particular, $I \neq 0$. Define as in Section 2 functions $f, \bar{f}, \bar{g}$ and $g$. Since $f$ satisfies (5) and $\bar{g}-\bar{f}$ is bounded, we obtain $\mu_{\Delta}(|g|>x) \sim x^{-2} l(x)$, where

$$
l(x)=\frac{I^{2} \ell^{2}}{2 \pi} .
$$

By Paragraph 3.1, the function $g$ is in the nonstandard domain of attraction of the normal law. More precisely, set

$$
L(x)=\frac{I^{2} \ell^{2}}{\pi} \log (x) \sim 2 \int_{1}^{x} \frac{l(u)}{u} .
$$

The functions $l$ and $L$ are the tail functions of $g$, as defined in Paragraph 3.1 .

Proposition 4.1 gives

$$
\int g\left(e^{i t G}-1\right)=(y-1) i t L(1 /|t|)+o(t L(1 /|t|))
$$

where $y=\frac{1}{1-\frac{3}{4} \log 3}$. Moreover, the function $g$ is locally Hölder on $\Delta$, by (9). Hence, all the assumptions of Theorem 3.4 are satisfied, for $a=y-1>0$. Let

$$
B_{n}=\sqrt{n \log n \frac{(2 y-1) I^{2} \ell^{2}}{2 \pi}},
$$

it satisfies $\frac{n}{B_{n}^{2}}(2 a+1) L\left(B_{n}\right) \rightarrow 1$. Hence, by Theorem 3.4 we obtain that $\frac{\sum_{k=0}^{n-1} g \circ U^{k}}{B_{n}} \rightarrow \mathcal{N}(0,1)$ in distribution with respect to $\mu_{\Delta}$. This is equivalent to the same convergence for $\bar{g}$, with respect to $\mu_{\bar{\Delta}}$, 
since $\bar{g}=g \circ \pi_{\Delta}$ and $\mu_{\Delta}=\left(\pi_{\Delta}\right)_{*}\left(\mu_{\bar{\Delta}}\right)$. Since $\bar{f}$ is cohomologous to $\bar{g}$, we get the same convergence for $\bar{f}$. Finally, since $\bar{f}=f \circ \pi_{X}$ and $\mu=\left(\pi_{X}\right)_{*}\left(\mu_{\bar{\Delta}}\right)$, we get that

$$
\frac{\sum_{k=0}^{n-1} f \circ T^{k}}{B_{n}} \rightarrow \mathcal{N}(0,1)
$$

on $X$, with respect to $\mu$.

The same argument applies to $\varphi_{+}-\int \varphi_{+}$, and we get that $\frac{\sum_{k=0}^{n-1} \varphi_{+} \circ T^{k}-n \int \varphi_{+}}{B_{n}}$ converges in distribution. Hence, Theorem 5.1 applies, with $b=1$.

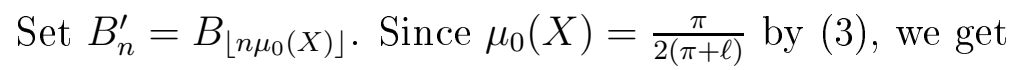

$$
B_{n}^{\prime} \sim \sqrt{n \log n \frac{(2 y-1) I^{2} \ell^{2}}{4(\pi+\ell)}} .
$$

Theorem 5.1 yields

$$
\frac{\sum_{k=0}^{n-1} f_{0} \circ T_{0}^{k}}{B_{n}^{\prime}} \rightarrow \mathcal{N}(0,1) .
$$

This concludes the proof of Theorem 1.1

\subsection{Proof of Theorem 1.3}

Let $f_{0}: X_{0} \rightarrow \mathbb{R}$ be Hölder continuous with $\int f_{0}=0$ and $I=0$. In this case, we can not use the cohomology trick any more, since the proofs of Lemmas 2.4 and 2.5 relied heavily on the property $(P 1)$. The argument will be to induce on the basis of the tower $\bar{\Delta}$, prove a central limit theorem here (using Gordin's martingale argument), and then get back to the original space by using Theorem 5.1] twice. The main difference in the inducing process with the previous paragraph is that we can no more apply Theorem 5.1 with $b=1$. Hence, we will need to prove that $\frac{1}{|n|^{b}} \sum_{k=0}^{n-1} f \circ T^{k}$ converges almost everywhere to 0 , for some $b<1$. Many arguments of this paragraph are strongly inspired by [You98, with additional technical complications due to the fact that our functions are not bounded.

Let $\bar{\Delta}_{0}$ be the basis of the tower $\bar{\Delta}$, and let $\bar{U}_{0}$ be the induced map on $\bar{\Delta}_{0}$ (with a return time $\varphi$ ). Define a new function $\bar{f}_{0}$ on $\bar{\Delta}_{0}$, by $\bar{f}_{0}(x)=\sum_{k=0}^{\varphi(x)-1} \bar{f}\left(\bar{U}^{k} x\right)$.

Lemma 5.3. There exists $\sigma_{0}^{2} \geqslant 0$ such that

$$
\frac{\sum_{k=0}^{n-1} \bar{f}_{0} \circ \bar{U}_{0}^{k}}{\sqrt{n}} \rightarrow \mathcal{N}\left(0, \sigma_{0}^{2}\right)
$$

Proof. Since $I=0$, it is not hard to check that there exists $\alpha_{1}<1$ such that $|f| \leqslant n^{\alpha_{1}}$ on the set of points bouncing $n$ times along the segments of the stadium. This implies that there exists $\varepsilon_{1}>0$ such that $f \in L^{2+\varepsilon_{1}}(X)$. Hence, $\bar{f} \in L^{2+\varepsilon_{1}}(\bar{\Delta})$. Since the return time $\varphi$ belongs to $L^{p}$ for all $p<\infty$, we get $\bar{f}_{0} \in L^{2+\varepsilon_{2}}\left(\bar{\Delta}_{0}\right)$ for some $\varepsilon_{2}>0$.

Let $\Delta_{0}$ be obtained by identifying the points on the same stable leaf. It is the basis of the expanding Young tower $\Delta$. Let $\pi_{0}: \bar{\Delta}_{0} \rightarrow \Delta_{0}$ be the canonical projection, and $U_{0}$ the dynamics induced by $\bar{U}_{0}$ on $\Delta_{0}$. Let $\mathcal{B}_{0}$ be the $\sigma$-algebra on $\bar{\Delta}_{0}$ obtained by pulling by $\pi_{0}$ the $\sigma$-algebra on $\Delta_{0}$. A measurable subset $B$ of $\bar{\Delta}_{0}$ is $\mathcal{B}_{0}$-measurable if, for almost all $x \in B$, the stable leaf through $x$ is contained in $B$. 
We will prove

$$
\sum_{n \geqslant 0}\left\|E\left(\bar{f}_{0} \mid \bar{U}_{0}^{n} \mathcal{B}_{0}\right)-\bar{f}_{0}\right\|_{L^{2}}<\infty
$$

and

$$
\sum_{n \geqslant 0}\left\|E\left(\bar{f}_{0} \mid \bar{U}_{0}^{-n} \mathcal{B}_{0}\right)\right\|_{L^{2}}<\infty
$$

By Gordin's Theorem [Gor69], this will imply the conclusion of the lemma.

The basis $\bar{\Delta}_{0}$ corresponds to a rectangle $R$ for the dynamics $T$, which is naturally partitioned as $R=\bigcup R_{i}$, where $R_{i}$ is an $s$-subrectangle of $R$. Let $\bar{\Delta}_{0, i}$ be the corresponding subset of $\bar{\Delta}_{0}$, so that $\left\{\bar{\Delta}_{0, i}\right\}$ gives a partition of $\bar{\Delta}_{0}$. Define a function $A: \bar{\Delta}_{0} \rightarrow \mathbb{R}$ by $A(x)=\sum_{k=0}^{\varphi(x)-1} \varphi_{+}\left(\pi_{X} \bar{U}^{k} x\right)$. It is constant on each set $\bar{\Delta}_{0, i}$, and corresponds to the number of times the original map $T_{0}$ is to be applied to $R_{i}$ so that this s-subrectangle makes a full (Markov) return to the base $R$. Since $\varphi$ belongs to every $L^{p}\left(\bar{\Delta}_{0}\right)$ for $p \geqslant 1$ and $\varphi_{+} \in L^{p}(X)$ for $1 \leqslant p<2$, the function $A$ belongs to $L^{p}\left(\bar{\Delta}_{0}\right)$ for $1 \leqslant p<2$. If $x, y$ are on the same unstable leaf in a rectangle $\bar{\Delta}_{0, i}$, we have

$$
\left|\bar{f}_{0}(x)-\bar{f}_{0}(y)\right| \leqslant C A(x) \tau^{s(x, y)}
$$

for some constant $C>0$ and some constant $\tau<1$. Here, $s(x, y)$ is the separation time of $x$ and $y$. Moreover, if $x, y$ are on the same stable leaf in a rectangle $\bar{\Delta}_{0, i}$,

$$
\left|\bar{f}_{0}(x)-\bar{f}_{0}(y)\right| \leqslant C A(x) d\left(\pi_{X} x, \pi_{X} y\right)^{\alpha} .
$$

for some $\alpha>0$.

Since the stable leaves are contracted at each iteration by at least $\lambda<1$, the atoms of the $\sigma$-algebra $\bar{U}_{0}^{n} \mathcal{B}_{0}$ have a diameter at most $C \lambda^{n}$. By (152), we get

$$
\left|\bar{f}_{0}(x)-E\left(\bar{f}_{0} \mid \bar{U}_{0}^{n} \mathcal{B}_{0}\right)(x)\right| \leqslant C A(x) \lambda^{\alpha n} .
$$

Unfortunately, $A$ does not belong to $L^{2}$, so a further argument is required to get (49). Let $p>0$ be such that $\frac{1}{p}+\frac{1}{2+\varepsilon_{2}}=\frac{1}{2}$. By $(\underline{53})$,

$$
1_{A \leqslant n^{2 p}}\left|\bar{f}_{0}(x)-E\left(\bar{f}_{0} \mid \bar{U}_{0}^{n} \mathcal{B}_{0}\right)(x)\right| \leqslant C n^{2 p} \lambda^{\alpha n} .
$$

Hence, this series is summable in $L^{2}$. Moreover,

$$
\left\|1_{A>n^{2 p}} \bar{f}_{0}\right\|_{L^{2}} \leqslant\left\|1_{A>n^{2 p}}\right\|_{L^{p}}\left\|\bar{f}_{0}\right\|_{L^{2+\varepsilon_{2}}} \leqslant \frac{\left(\int A\right)^{1 / p}}{n^{2}}\left\|\bar{f}_{0}\right\|_{L^{2+\varepsilon_{2}}} .
$$

The function $E\left(\bar{f}_{0} \mid \bar{U}_{0}^{n} \mathcal{B}_{0}\right)$ is bounded in $L^{2+\varepsilon_{2}}$ by $\left\|\bar{f}_{0}\right\|_{L^{2+\varepsilon_{2}}}$. Hence, we obtain

$$
\left\|1_{A>n^{2 p}}\left|\bar{f}_{0}-E\left(\bar{f}_{0} \mid \bar{U}_{0}^{n} \mathcal{B}_{0}\right)\right|\right\|_{L^{2}}=O\left(1 / n^{2}\right),
$$

which is summable. This proves (49).

Let $\bar{h}=E\left(\bar{f}_{0} \mid \mathcal{B}_{0}\right)$. This function is constant along the stable leaves, and has zero integral (since $\bar{f}_{0}$ also has zero integral). Hence, it induces a function $h$ on the quotient $\Delta_{0}$. Since $\bar{f}_{0} \in L^{2}$, it satisfies $h \in L^{2}\left(\Delta_{0}\right)$. The following lemma is an easy consequence of the Hölder properties of the invariant measure and (51), see [You98, Sublemma page 612] for details. 
Lemma 5.4. There exists constants $C>0$ and $\tau<1$ such that, for all $x, y$ in the same unstable leaf of a set $\bar{\Delta}_{0, i}$,

$$
|\bar{h}(x)-\bar{h}(y)| \leqslant C A(x) \tau^{s(x . y)}
$$

The function $A$ is integrable. Hence, by Gou04, Lemma 3.4], this implies that the function $\widehat{U}_{0} h$ is Hölder continuous on $\Delta_{0}$. By Gou04, Corollary 3.3], we get:

$$
\widehat{U}_{0}^{n} h \text { tends exponentially fast to } 0 \text { in the space of Hölder continuous functions on } \Delta_{0} \text {. }
$$

A computation gives

$$
\left\|E\left(\bar{f}_{0} \mid \bar{U}_{0}^{-n} \mathcal{B}_{0}\right)\right\|_{L^{2}}^{2}=\int h \cdot\left(\widehat{U}_{0}^{n} h\right) \circ U_{0}^{n} \leqslant\|h\|_{L^{2}}\left\|\left(\widehat{U}_{0}^{n} h\right) \circ U_{0}^{n}\right\|_{L^{2}}=\|h\|_{L^{2}}\left\|\widehat{U}_{0}^{n} h\right\|_{L^{2}}
$$

Hence, this term is exponentially small. This proves (50) and concludes the proof of Lemma 5.3

The return time $\varphi$ also satisfies a central limit theorem, by the same argument. Hence, by Theorem 5.1 (applied with $b=1$ ), there exists $\sigma_{1}^{2} \geqslant 0$ such that

$$
\frac{\sum_{k=0}^{n-1} \bar{f} \circ \bar{U}^{k}}{\sqrt{n}} \rightarrow \mathcal{N}\left(0, \sigma_{1}^{2}\right)
$$

Going from $\bar{\Delta}$ to $X$, it implies that

$$
\frac{\sum_{k=0}^{n-1} f \circ T^{k}}{\sqrt{n}} \rightarrow \mathcal{N}\left(0, \sigma_{1}^{2}\right)
$$

Moreover, the return time $\varphi_{+}: X \rightarrow \mathbb{N}$ satisfies a limit theorem with normalization $\sqrt{n \log n}$. Since $\sqrt{n}=o(\sqrt{n \log n})$, we can unfortunately not apply Theorem 5.1 with $b=1$. However, if we can prove the following lemma, then this theorem applies with $b<1$, and this concludes the proof of Theorem 1.3.

Lemma 5.5. For all $b>1 / 2$,

$$
\frac{1}{|n|^{b}} \sum_{k=0}^{n-1} f \circ T^{k} \rightarrow 0
$$

almost everywhere in $X$ when $n \rightarrow \pm \infty$.

Proof. We first estimate the decay of correlations of $\bar{f}_{0}$ for $\bar{U}_{0}$. We will use the notations of the proof of Lemma 5.3. We have

$$
\int \bar{f}_{0} \cdot \bar{f}_{0} \circ \bar{U}_{0}^{2 n}=\int \bar{f}_{0} \cdot E\left(\bar{f}_{0} \circ \bar{U}_{0}^{n} \mid \mathcal{B}_{0}\right) \circ \bar{U}_{0}^{n}+\int \bar{f}_{0} \cdot\left(\bar{f}_{0} \circ \bar{U}_{0}^{2 n}-E\left(\bar{f}_{0} \circ \bar{U}_{0}^{n} \mid \mathcal{B}_{0}\right) \circ \bar{U}_{0}^{n}\right) .
$$

The contraction properties of $\bar{U}_{0}$ along stable manifolds and (52) give $\left|\bar{f}_{0} \circ \bar{U}_{0}^{n}(x)-E\left(\bar{f}_{0} \circ \bar{U}_{0}^{n} \mid \mathcal{B}_{0}\right)(x)\right| \leqslant$ $C A\left(\bar{U}_{0}^{n} x\right) \lambda^{\alpha n}$. Hence, the second integral in (56) is at most

$$
\int\left|\bar{f}_{0}\right| \cdot A \circ \bar{U}_{0}^{2 n} \lambda^{\alpha n} \leqslant\left\|\bar{f}_{0}\right\|_{L^{2+\varepsilon_{2}}}\|A\|_{L^{p}} \lambda^{\alpha n}
$$

where $p<2$ is chosen so that $\frac{1}{2+\varepsilon_{2}}+\frac{1}{p}=1$. Hence, this term decays exponentially fast. 
In the first integral of (56), the function $E\left(\bar{f}_{0} \circ \bar{U}_{0}^{n} \mid \mathcal{B}_{0}\right) \circ \bar{U}_{0}^{n}$ is $\mathcal{B}_{0}$-measurable (i.e., constant along stable leaves). Hence, this integral is equal to

$$
\int \bar{h} \cdot E\left(\bar{f}_{0} \circ \bar{U}_{0}^{n} \mid \mathcal{B}_{0}\right) \circ \bar{U}_{0}^{n}
$$

Let $\bar{h}_{n}=E\left(\bar{f}_{0} \circ \bar{U}_{0}^{n} \mid \mathcal{B}_{0}\right)$, it is $\mathcal{B}_{0}$-measurable and defines a function $h_{n}$ on the quotient $\Delta_{0}$. The integral (57) is then equal to

$$
\int_{\Delta_{0}} h \cdot h_{n} \circ U_{0}^{n}=\int \widehat{U}_{0}^{n} h \cdot h_{n}
$$

The $L^{2}$-norm of $h_{n}$ is bounded independently of $n$. By (54), (58) is exponentially small. This proves that $\int \bar{f}_{0} \cdot \bar{f}_{0} \circ \bar{U}_{0}^{2 n}$ decays exponentially. In the same way, $\int \bar{f}_{0} \cdot \bar{f}_{0} \circ \bar{U}_{0}^{2 n+1}$ decays exponentially.

Since the correlations of $\bar{f}_{0}$ decay exponentially fast and $\bar{f}_{0} \in L^{2}$, [Kac96, Theorem 16] implies that $\frac{1}{n^{b}} \sum_{k=0}^{n-1} \bar{f}_{0} \circ \bar{U}_{0}^{k}$ tends to zero almost everywhere when $n \rightarrow+\infty$, for all $b>1 / 2$.

Now to see that $\frac{1}{n^{b}} \sum_{k=0}^{n-1} \bar{f} \circ \bar{U}^{k}$ tends to zero almost everywhere in $\bar{\Delta}$ when $n \rightarrow+\infty$, for all $b>1 / 2$, we use [MT04, Lemma 2.1 (a)] which gives this convergence on $\bar{\Delta}_{0}$. However, by the ergodicity of $\bar{U}$, the set on which this convergence holds must have either full or zero measure. As $\bar{\Delta}_{0}$ has positive measure, we get this convergence almost everywhere on $\bar{\Delta}$. Finally, this implies the same for $f$ in $X$. We have proved (55) for any $b>1 / 2$ when $n \rightarrow+\infty$.

To deal with $n \rightarrow-\infty$, we go to the natural extension. It is sufficient to prove the result for $\bar{f}_{0}$ in $\bar{\Delta}_{0}$, since the previous reasoning still applies (using the fact that the natural extension is functorial, i.e., the natural extension commutes with induction and projections). In the natural extension $\bar{\Delta}_{0}^{\prime}$ of $\bar{\Delta}_{0}$, we have $\int \bar{f}_{0}^{\prime} \cdot \bar{f}_{0}^{\prime} \circ \bar{U}_{0}^{\prime-n}=\int \bar{f}_{0} \circ \bar{U}_{0}^{n} \cdot \bar{f}_{0}$, which is exponentially small. Hence, Kac96, Theorem 16] still applies and gives the desired result.

Remark 5.6. As $\mu_{0}(X)>0$, we may apply [MT04, Lemma 2.1 (a)] just as we did in the proof above to see that Lemma 5.5 implies

$$
\frac{1}{|n|^{b}} \sum_{k=0}^{n-1} f_{0} \circ T_{0}^{k} \rightarrow 0
$$

almost everywhere when $n \rightarrow \pm \infty$, for any $b>1 / 2$.

\section{Acknowledgements}

We are much grateful to D. Szász and T. Varjú for useful discussions and for their valuable remarks on earlier versions of the manuscript. This paper has grown out of discussions we had at the CIRM conference on multi-dimensional non-uniformly hyperbolic systems in Marseille in May 2004, and while S. G. visited the Institute of Mathematics of the BUTE in October 2004. The hospitality of both institutions, along with the financial support of Hungarian National Foundation for Scientific Research (OTKA), grants T32022 and TS040719 is thankfully acknowledged.

\section{A Proof of Lemma 3.6}

Let $U_{0}$ be the map induced by $U$ on the basis $\Delta_{0}$ of the tower. Denote by $\varphi$ the first return time on the basis, so that $U_{0}(x)=U^{\varphi(x)}(x)$. Note that $\varphi(x)$ can also be defined for $x \in \Delta \backslash \Delta_{0}$ as the first hitting time of the basis. 
Let $F$ be a finite subset of $\mathbb{N}$. Let $\left(n_{i}\right)_{i \in F}$ be positive integers. Let

$$
K\left(F, n_{i}\right)=\left\{x \in \Delta_{0} \mid \forall i \in F, \varphi\left(U_{0}^{i} x\right)=n_{i}\right\} .
$$

Lemma A.1. There exists a constant $C$ such that, for all $F$ and $n_{i}$ as above,

$$
\mu_{\Delta}\left(K\left(F, n_{i}\right)\right) \leqslant \prod_{i \in F}\left(C \rho^{n_{i}}\right)
$$

Proof. The proof is by induction on $\max F$, and the result is trivial when $F=\emptyset$. Write $F^{\prime}=$ $\{i-1 \mid i \in F, i \geqslant 1\}$ and, for $i \in F^{\prime}$, set $n_{i}^{\prime}=n_{i+1}$.

if $0 \notin F, K\left(F, n_{i}\right)=U_{0}^{-1}\left(K\left(F^{\prime}, n_{i}^{\prime}\right)\right)$. Since $U_{0}$ preserves $\mu_{\Delta}$ and $\max F^{\prime}<\max F$, we get the result. Otherwise, $0 \in F$. Then $K\left(F, n_{i}\right)=U_{0}^{-1}\left(K\left(F^{\prime}, n_{i}^{\prime}\right)\right) \cap\left\{x \in \Delta_{0}, \varphi(x)=n_{0}\right\}$. By bounded distortion, we get

$$
\mu_{\Delta}\left(K\left(F, n_{i}\right)\right) \leqslant C \mu_{\Delta}\left(K\left(F^{\prime}, n_{i}^{\prime}\right)\right) \mu_{\Delta}\left\{x \in \Delta_{0}, \varphi(x)=n_{0}\right\} \leqslant C \mu_{\Delta}\left(K\left(F^{\prime}, n_{i}^{\prime}\right)\right) \rho^{n_{0}} .
$$

Lemma A.2. There exist $C>0$ and $\theta<1$ such that, for all $n \in \mathbb{N}$,

$$
\int_{U^{-n} \Delta_{0}} \tau^{\Psi_{n}} \leqslant C \theta^{n}
$$

Proof. Let $\kappa>0$ be very small (how small will be specified later in the proof). Then

$$
U^{-n} \Delta_{0} \subset\left\{x \in \Delta \mid \Psi_{n}(x) \geqslant \kappa n\right\} \cup\{x \in \Delta \mid \varphi(x) \geqslant n / 2\} \cup\left\{x \in \Delta \mid \varphi(x)<n / 2, \Psi_{n}(x)<\kappa n\right\} .
$$

On the first of these sets, $\tau^{\Psi_{n}} \leqslant \tau^{\kappa n}$, whence the integral of $\tau^{\Psi_{n}}$ is exponentially small. The second of these sets has exponentially small measure. Finally, the last of these sets is contained in $\bigcup_{i=0}^{n / 2} U^{-i} \Gamma_{n}$, where

$$
\Gamma_{n}=\left\{x \in \Delta_{0} \mid \sum_{0 \leqslant i \leqslant \kappa n} \varphi\left(U_{0}^{i} x\right) \geqslant n / 2\right\} .
$$

To conclude the proof of the Lemma, it is sufficient to prove that the measure of $\Gamma_{n}$ is exponentially small.

Take $L \in \mathbb{N}$ such that $\forall n \geqslant L,(C \rho)^{n} \leqslant \rho^{n / 2}$, where $C$ is the constant given by Lemma A.1. For $x \in \Gamma_{n}$, let $F(x):=\left\{0 \leqslant i \leqslant \kappa n \mid \varphi\left(U_{0}^{i} x\right) \geqslant L\right\}$. Then

$$
\sum_{i \in F(x)} \varphi\left(U_{0}^{i} x\right) \geqslant \frac{n}{2}-\sum_{i \notin F(x)} L \geqslant(1 / 2-L \kappa) n .
$$

This implies that

$$
\Gamma_{n} \subset \bigcup_{F \subset[0,\lfloor\kappa n]]} \bigcup_{\substack{n_{i} \geqslant L \\ \sum_{i \in F} n_{i} \geqslant(1 / 2-L \kappa) n}} K\left(F, n_{i}\right) .
$$

By Lemma A.1, we get

$$
\begin{aligned}
\mu_{\Delta}\left(\Gamma_{n}\right) & \leqslant \sum_{F \subset[0,\lfloor\kappa n\rfloor]} \sum_{\substack{n_{i} \geqslant L \\
\sum_{i \in F} \geqslant(1 / 2-L \kappa) n}} \prod_{i \in F}\left(C \rho^{n_{i}}\right) \leqslant \sum_{k=0}^{\lfloor\kappa n\rfloor}\left(\begin{array}{c}
\lfloor\kappa n\rfloor \\
k
\end{array}\right) \sum_{\substack{n_{i}, \ldots, n_{k-1} \geqslant L \\
\sum n_{i} \geqslant(1 / 2-L \kappa) n}}\left(C \rho^{n_{0}}\right) \ldots\left(C \rho^{n_{k-1}}\right) \\
& \leqslant 2^{\kappa n} \sum_{\substack { 0 \leqslant k \leqslant \kappa n \\
\begin{subarray}{c}{n_{0}, \ldots, n_{k-1} \geqslant L \\
n_{i} \geqslant(1 / 2-L \kappa) n{ 0 \leqslant k \leqslant \kappa n \\
\begin{subarray} { c } { n _ { 0 } , \ldots , n _ { k - 1 } \geqslant L \\
n _ { i } \geqslant ( 1 / 2 - L \kappa ) n } }\end{subarray}} \sum^{\sum n_{i} / 2} \leqslant 2^{\kappa n} \sum_{\substack{0 \leqslant k \leqslant \kappa n \\
0}} \sum_{\substack{n_{0}, \ldots, n_{k-1} \in \mathbb{N} \\
\sum n_{i} \geqslant(1 / 2-L \kappa) n}} \rho^{\sum n_{i} / 2} .
\end{aligned}
$$


For $r \in \mathbb{N}$

$$
\sum_{n_{0}+\cdots+n_{k-1}=r} \rho^{\sum n_{i} / 2}=\rho^{r / 2} \operatorname{Card}\left\{n_{0}, \ldots, n_{k-1} \mid \sum n_{i}=r\right\}=\rho^{r / 2}\left(\begin{array}{c}
r+k \\
k
\end{array}\right) \leqslant \rho^{r / 2} \frac{(r+k)^{k}}{k !} .
$$

Hence,

$$
\mu_{\Delta}\left(\Gamma_{n}\right) \leqslant 2^{\kappa n} \sum_{0 \leqslant k \leqslant \kappa n} \sum_{r \geqslant(1 / 2-L \kappa) n} \rho^{r / 2} \frac{(r+k)^{k}}{k !} .
$$

The sequence $u_{r}=\rho^{r / 2} \frac{(r+k)^{k}}{k !}$ satisfies $\frac{u_{r+1}}{u_{r}} \leqslant \rho^{\prime}:=\rho^{1 / 2} e^{\frac{\kappa}{1 / 2-L \kappa}}$ for all $r \geqslant(1 / 2-L \kappa) n$ and $k \leqslant \kappa n$. if $\kappa$ is small enough, $\rho^{\prime}<1$, and we get

$$
\mu_{\Delta}\left(\Gamma_{n}\right) \leqslant 2^{\kappa n} \sum_{0 \leqslant k \leqslant \kappa n} \rho^{(1 / 2-L \kappa) n / 2} \frac{((1 / 2-L \kappa) n+\kappa n)^{k}}{k !} \frac{1}{1-\rho^{\prime}} \leqslant \frac{2^{\kappa n}}{1-\rho^{\prime}} \rho^{(1 / 2-L \kappa) n / 2} \sum_{0 \leqslant k \leqslant \kappa n} \frac{n^{k}}{k !} .
$$

The sequence $\frac{n^{k}}{k !}$ is increasing for $k \leqslant n$. Hence, we finally get

$$
\mu_{\Delta}\left(\Gamma_{n}\right) \leqslant \frac{2^{\kappa n}}{1-\rho^{\prime}} \rho^{(1 / 2-L \kappa) n / 2}(\kappa n+1) \frac{n^{\lfloor\kappa n\rfloor}}{\lfloor\kappa n\rfloor !} .
$$

Using Stirling's Formula, it is easy to check that this expression is exponentially small if $\kappa$ is small enough. This concludes the proof.

Proof of Lemma 3.6, Let $\theta$ be given by Lemma A.2. Choose $\alpha>0$ so that $e^{\varepsilon \alpha} \theta<1$. Then

$$
U^{-n} \Delta_{0} \subset\{x \in \Delta \mid \omega(x) \geqslant \alpha n\} \cup\left[\{x \in \Delta \mid \omega(x)<\alpha n\} \cap U^{-n} \Delta_{0}\right] .
$$

Hence,

$$
\int_{U^{-n} \Delta_{0}} e^{\varepsilon \omega} \tau^{\Psi_{n}} \leqslant \int_{\omega \geqslant \alpha n} e^{\varepsilon \omega}+e^{\varepsilon \alpha n} \int_{U^{-n} \Delta_{0}} \tau^{\Psi_{n}} .
$$

The first term is exponentially small since $e^{\varepsilon} \rho<1$. Lemma A.2 and the definition of $\alpha$ also imply that the second term is exponentially small.

\section{References}

[Aar97] Jon Aaronson. An introduction to infinite ergodic theory, volume 50 of Mathematical Surveys and Monographs. American Mathematical Society, 1997.

[AD01] Jon Aaronson and Manfred Denker. A local limit theorem for stationary processes in the domain of attraction of a normal distribution. In N. Balakrishnan, I.A. Ibragimov, and V.B. Nevzorov, editors, Asymptotic methods in probability and statistics with applications. Papers from the international conference, St. Petersburg, Russia, 1998, pages 215-224. Birkhäuser, 2001.

[Bun79] Leonid Bunimovich. On the ergodic properties of nowhere dispersing billiards. Comm. Math. Phys., 65:295-312, 1979. 
[BY93] Viviane Baladi and Lai-Sang Young. On the spectra of randomly perturbed expanding maps. Comm. Math. Phys., 156:355-385, 1993.

[Che97] Nikolai Chernov. Entropy, Lyapunov exponents and mean free path for billiards. J. Statist. Phys., 88:1-29, 1997.

[Che99] Nikolai Chernov. Decay of correlations and dispersing billiards. J. Statist. Phys., 94:513-556, 1999.

[CZ] Nikolai Chernov and Hongkun Zhang. Billiards with polynomial mixing rates. Preprint.

[Eag76] G. K. Eagleson. Some simple conditions for limit theorems to be mixing. Teor. Verojatnost. i Primenen., 21(3):653-660, 1976.

[Gor69] Mikhail Gordin. The central limit theorem for stationary processes. Dokl. Akad. Nauk SSSR, 188:739-741, 1969.

[Gou03] Sébastien Gouëzel. Statistical properties of a skew-product with a curve of neutral points. Preprint, 2003.

[Gou04] Sébastien Gouëzel. Central limit theorem and stable laws for intermittent maps. Probab. Theory and Rel. Fields, 128:82-122, 2004.

[Hen93] Hubert Hennion. Sur un théorème spectral et son application aux noyaux lipschitziens. Proc. Amer. Math. Soc., 118:627-634, 1993.

[Kac96] A. G. Kachurovskiı. Rates of convergence in ergodic theorems. Russian Math. Surveys, 51:653-703, 1996.

[KL99] Gerhard Keller and Carlangelo Liverani. Stability of the spectrum for transfer operators. Ann. Scuola Norm. Sup. Pisa Cl. Sci. (4), 28(1):141-152, 1999.

[Mac83] J. Machta. Power law decay of correlations in a billiard problem. J. Statist. Phys., 32:555564, 1983.

[Mar04] Roberto Markarian. Billiards with polynomial decay of correlations. Ergodic Theory Dynam. Systems, 24:177-197, 2004.

[MT04] Ian Melbourne and Andrew Török. Statistical limit theorems for suspension flows. Israel J. Math., pages 191-210, 2004.

[SV] Domokos Szász and Tamás Varjú. in preparation.

[SV04a] Domokos Szász and Tamás Varjú. Local limit theorem for the Lorentz process and its recurrence on the plane. Ergodic Theory Dynam. Systems, 24:257-278, 2004.

[SV04b] Domokos Szász and Tamás Varjú. Markov towers and stochastic properties of billiards. In Modern dynamical systems and applications, pages 433-445. Cambridge University Press, 2004.

[You98] Lai-Sang Young. Statistical properties of dynamical systems with some hyperbolicity. Ann. of Math. (2), 147:585-650, 1998.

[You99] Lai-Sang Young. Recurrence times and rates of mixing. Israel J. Math., 110:153-188, 1999. 